\title{
Investigation of Plasma Assisted Combustion in an Internal Combustion Engine
}

by

\author{
Nicholas A. E. Giordano
}

\author{
A Thesis submitted to \\ the Faculty of Graduate Studies and Research \\ in partial fulfilment of \\ the requirements for the degree of \\ Master of Applied Science \\ Ottawa-Carleton Institute for \\ Mechanical and Aerospace Engineering
}

Department of Mechanical and Aerospace Engineering

Carleton University

Ottawa, Ontario, Canada

December 2012

Copyright $(C)$

2012 - Nicholas A. E. Giordano 
Library and Archives

Canada

Published Heritage

Branch

395 Wellington Street

Ottawa ON K1A ON4

Canada
Bibliothèque et

Archives Canada

Direction du

Patrimoine de l'édition

395 , rue Wellington

Ottawa ON K1A ON4

Canada
Your file Votre référence

ISBN: 978-0-494-94245-1

Our file Notre référence

ISBN: 978-0-494-94245-1
NOTICE:

The author has granted a nonexclusive license allowing Library and Archives Canada to reproduce, publish, archive, preserve, conserve, communicate to the public by telecommunication or on the Internet, loan, distrbute and sell theses worldwide, for commercial or noncommercial purposes, in microform, paper, electronic and/or any other formats.

The author retains copyright ownership and moral rights in this thesis. Neither the thesis nor substantial extracts from it may be printed or otherwise reproduced without the author's permission.
AVIS:

L'auteur a accordé une licence non exclusive permettant à la Bibliothèque et Archives Canada de reproduire, publier, archiver, sauvegarder, conserver, transmettre au public par télécommunication ou par l'Internet, prêter, distribuer et vendre des thèses partout dans le monde, à des fins commerciales ou autres, sur support microforme, papier, électronique et/ou autres formats.

L'auteur conserve la propriété du droit d'auteur et des droits moraux qui protege cette thèse. $\mathrm{Ni}$ la thèse ni des extraits substantiels de celle-ci ne doivent être imprimés ou autrement reproduits sans son autorisation.
In compliance with the Canadian Privacy Act some supporting forms may have been removed from this thesis.

While these forms may be included in the document page count, their removal does not represent any loss of content from the thesis.
Conformément à la loi canadienne sur la protection de la vie privée, quelques formulaires secondaires ont été enlevés de cette thèse.

Bien que ces formulaires aient inclus dans la pagination, il n'y aura aucun contenu manquant. 


\section{Abstract}

This research evaluates the effect on combustion cylinder pressure due to ignition from a $30 \mathrm{kHz}$ plasma ignition device, coupled with an arc discharge, in an internal combustion engine. The work includes outfitting an internal combustion engine with an optical encoder for refined timing control, and sensors for monitoring internal cylinder pressure and other engine parameters. Capturing and analyzing the collected data was performed by using multiple National Instruments data acquisition boards and mathematical evaluation software such as MATLAB. For each set of experimental parameters, over 1000 pressure curves were collected and a statistical analysis was conducted on the datasets.

The experiments were motivated by a hypothesis of the ignition system developer that the use of plasma at the ignition site results in the formation of radicals, which are expected to change the ignition delay time and flame propagation characteristics.

The experimental results show that the plasma ignition device creates two plasma states, one postulated to be in a streamer discharge state and the other in a glow discharge state. It was postulated by the industrial partner that an improvement of $4 \%$ to $9 \%$ in engine performance is possible through use of the spatial plasma discharge ignition device, however the experiment designed here provided a peak error 
of $14.4 \%$ which is beyond the expected difference seen. However recommendations for improvement upon these errors (systematic and random) are discussed. The results from the engine experiments show that the number of voltage pulses and the amount of current induced during each pulse has no effect on the pressure traces to within the experimental uncertainty of the apparatus. However, the comparison of the plasma discharge ignition system to an inductive discharge (ID) unit shows that for engine loads of $60 \%$, the peak cylinder pressure increases with breakdown current, and thus the conventional inductive discharge system creates higher cylinder pressures compared to the spatial plasma discharge ignition (SPDI) device under investigation. However, the mass fraction burn analyses conducted on these results show that the difference in combustion rates and ignition delay times are within the experimental uncertainty and are therefore not statistically significant. 


\section{Acknowledgments}

I would like to thank my supervisor, Ronal E. Miller, for making this project possible and providing me with the guidance and insight necessary to complete it. I would like to thank Mr. Glen Clarke and Dr. Alex Plotnikov, from Sphenic Technologies Inc., for their financial and in-kind contributions towards the project and towards the MITACS accelerate program. In addition, I would like to thank the thesis review committee for their time and efforts. Without the contributions of the above mentioned, this project would not have been possible.

As well I would like to thank, my family for their endless support, encouragement and inspiration to pursue graduate studies and research. I would like to thank, in particular, my parents for their generous financial support throughout my university career, without you this would not have been possible. 


\section{Table of Contents}

$\begin{array}{ll}\text { Abstract } & \text { iii }\end{array}$

Acknowledgments v v

Table of Contents vi vi

List of Tables $\quad$ xii

List of Figures $\quad$ xiii

List of Acronyms $\quad$ xxii

List of Symbols $\quad$ xxiv

1 Introduction 1

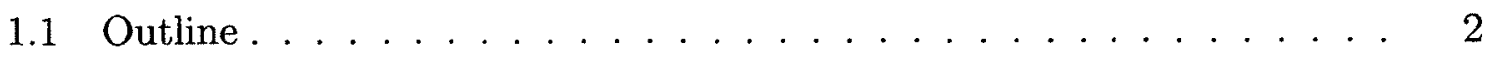

1.1.1 Background Information and Literature Review . . . . . . . 2

1.1.2 Imaging the Spark Propagation . . . . . . . . . . . . . . 2

1.1.3 Experimental Methods ............... 3

1.1.4 Results and Discussions ................ 4 
2 Background Information and Relevant Literature 5

2.1 Plasma Ionization and Molecular Interactions $\ldots \ldots \ldots$

2.1.1 General Atom or Molecule Excitation . . . . . . . . . . . . 5

2.1.2 Molecular Interaction Methods . . . . . . . . . . . . 13

2.1.3 Positive Ion Chemical Reactions and Recombination . . . . 15

2.1.4 Electron Emissions in Plasma States and Electric Fields . . . 17

2.2 Relevant Literature in Plasma Discharge Ignition Systems _ . . . . 19

2.3 The Plasma Settings and Parameters . . . . . . . . . . . 21

2.3.1 Issues Resulting From Expected Curves and Actual Curves . . 25

3 Combustion and Internal Combustion Engines $\quad 27$

3.1 Engine Geometry . . . . . . . . . . . . . . . . . . 27

3.1 .1 Piston Position . . . . . . . . . . . . . . . . 29

3.1 .2 Cylinder Volume . . . . . . . . . . . . . . . . . . 29

3.1.3 Piston Speed and Acceleration . . . . . . . . . . . . 30

3.2 Engine Work . . . . . . . . . . . . . . . . . . 32

3.3 Mass Fraction Burn Approximation . . . . . . . . . . . . . . . 34

3.3 .1 Method $1 \ldots \ldots \ldots \ldots . \ldots \ldots$

3.3 .2 Method $2 \ldots \ldots \ldots \ldots \ldots \ldots \ldots$

3.3 .3 Method $3 \ldots \ldots \ldots \ldots \ldots$

3.3 .4 Conclusions . . . . . . . . . . . . . . . . 38

4 Experimental Methods and Results for Imaging the Ignition Systems 41

4.1 Purpose of Experiment . . . . . . . . . . . . . . 41

vii 
4.2 Apparatuses of Experiments . . . . . . . . . . . . . . . 42

4.2 .1 Ambient Pressure Experiment . . . . . . . . . . . . . . 42

4.2 .2 Pressurized Experiment $\ldots \ldots \ldots \ldots$

4.3 Ambient Pressure . . . . . . . . . . . . . . . . . . . . 44

4.4 Discharge at Pressures Greater Than Atmospheric . . . . . . . . . 46

4.4.1 Breakdown Current Versus Pressure . . . . . . . . . . 46

4.4.2 Constant Plasma Settings With Incremental increases in Pressure 48

4.5 Conclusions . . . . . . . . . . . . . . . 57

5 Internal Combustion Engine Experimental Methods 58

5.1 Experiment Apparatus . . . . . . . . . . . . . . . 58

5.1 .1 Engine Timing . . . . . . . . . . . . . 60

5.1 .2 Air and Fuel Management . . . . . . . . . . . . 64

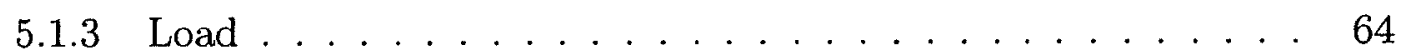

5.1 .4 Pressure Measurements . . . . . . . . . . . . . . 65

5.1 .5 Ignition Coils $\ldots \ldots \ldots \ldots \ldots \ldots$

5.1 .6 Oscilloscope Data Acquisition . . . . . . . . . . . 67

5.2 Motored Pressure Acquisition and Top Dead Centre Measurements . 68

5.2 .1 Motored Pressure Setup . . . . . . . . . . . . . . . 69

5.2.2 Top Dead Centre Measurement Methods . . . . . . . . . 70

5.3 Other Acquisition Techniques and Procedures . . . . . . . . . 73

5.3.1 Acquisition of Signals and Noise Reduction . . . . . . . . 73

5.3 .2 Signal Shielding . . . . . . . . . . . . . . . . 74

5.4 Systematic Error and Instrument Hysteresis . . . . . . . . . . 74 
6 Post-Processing Techniques $\quad 76$

6.1 Engine Control Program and Initial Acquisition . . . . . . . . . . 77

6.1.1 Engine Timing Configuration . . . . . . . . . . . . 77

6.1.2 Sampling Rates and Synchronization . . . . . . . . . 78

6.1 .3 Acquisition .................. 78

6.2 MATLAB Post-Processing Techniques . . . . . . . . . . . . . 79

6.2.1 Filtering and Signal Analysis . . . . . . . . . . . 79

6.2.2 Program Process ................. . 81

6.3 Real-Time LabVIEW Engine Control, Acquisition and Performance Analysis ...................... . . 82

6.4 Statistical Analysis of Curves . . . . . . . . . . . . . . 83

7 Results and Discussion of Internal Combustion Engine Pressure $\begin{array}{ll}\text { Measurements } & 90\end{array}$

7.1 Varying SPDI Parameters . . . . . . . . . . . . . . 91

7.1.1 Changes in Plasma Duration . . . . . . . . . . . . . . 91

7.1.2 Changes in Plasma Power . . . . . . . . . . . . . 96

7.2 Spatial Plasma Discharge Ignition Versus Conventional Inductive Discharge Ignition . . . . . . . . . . . . . . 100

7.3 Two Tail T-Test . . . . . . . . . . . . . . 105

7.3.1 Methods ................. 105

7.3 .2 Choosing $d_{0} \ldots \ldots \ldots . \ldots \ldots 6$

7.3 .3 T-Test Results . . . . . . . . . . . . . 107

7.4 The SPDI Spark Profile . . . . . . . . . . . . 110 
7.5 Engine Experiment Conclusions . . . . . . . . . . . . 110

8 Conclusion $\quad 112$

8.1 Summary . . . . . . . . . . . . . . . . . . . 112

8.1.1 Imaging the Ignition Systems $\ldots \ldots \ldots \ldots \ldots$

8.1.2 Single Cylinder Engine Pressure Trace Analysis . . . . . . . . 113

8.1 .3 Future Work . . . . . . . . . . . . . . . . . . . . 114

A Instrumentation Information $\quad 116$

A.1 Optrand AutoPSI-S fibre Optic Pressure Sensor . . . . . . . . 117

A.2 MKII Nanosense High Speed Camera . . . . . . . . . . . . . 118

A.3 SPDI User Interface . . . . . . . . . . . . . . . . . . . . 119

$\begin{array}{ll}B \text { EngineWiring } & 120\end{array}$

B.1 Data Acquisition Circuits $\ldots \ldots \ldots \ldots \ldots \ldots$

B.2 Coil Circuits . . . . . . . . . . . . . . . . . . . . . 122

B.3 Power Supply Circuits . . . . . . . . . . . . . . . . . . . . . 122

B.4 Physical Coils Used in Acquiring Voltage and Current . . . . . . . 123

C Data Processing $\quad 124$

C.1 MATLAB Flow Chart . . . . . . . . . . . . . . 125

C.2 MATLAB Database GUI Front Panel . . . . . . . . . . . . . 126

C.3 Pressure Filter and Residuals . . . . . . . . . . . . . 126

C.4 Pressure Filter and Residuals . . . . . . . . . . . . . 127

C.5 Statistical Analyses - Supporting Figures . . . . . . . . . . 128 
D Results and Discussion: Supporting Figures 130

D.1 Pressure Traces for Variable Spark Duration . . . . . . . . . . . 131

D.2 Pressure Traces for Variable Spark Plasma Power . . . . . . . . . . 133

D.3 Pressure Traces for Variable Breakdown Current . . . . . . . . . 135

$\begin{array}{ll}\text { List of References } & 136\end{array}$ 


\section{List of Tables}

2.1 Table of Spark Parameters, symbols and physical configuration of SPDI system hardware. . . . . . . . . . . . . . . 23

3.1 List of engine geometry parameters and their values. . . . . . . . . . 28

7.1 Measured spark currents for two SPDI system settings and the conventional ID system . . . . . . . . . . . . . . . . . . 100

7.2 Maximum cylinder pressures achieved by ignition from two SPDI spark profiles and the conventional ID system . . . . . . . . . . . . 101

7.3 Results of T-test statistic comparing ID to both SPDI Profile 1 and SPDI Profile 2 trials. For each interval of rejection, the curves are said to be statistically NOT EQUIVALENT . . . . . . . . . . . . . . . 109

A.1 Calibration specifications and details pertaining to the Optrand AutoPSI-S fibre optic pressure transducer. . . . . . . . . . . . . . . 117 


\section{List of Figures}

2.1 Bohr model of an atom with its nucleus at the centre and an electron dropping from its outer most orbital to the second orbital followed by

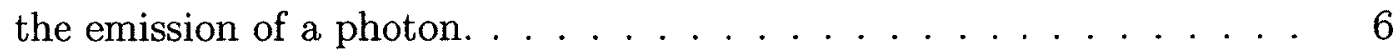

2.2 A two dimensional representation of the volume limit in which the electrons can be found for a single $2 p$ type orbital. . . . . . . . . 7

2.3 Molecular Orbital (MO) diagram for the triplet oxygen molecular state. 10

2.4 Molecular Orbital (MO) diagram for the lowest energy singlet state of molecular oxygen. . . . . . . . . . . . . . 10

2.5 Molecular Orbital (MO) diagram for the second energy singlet state

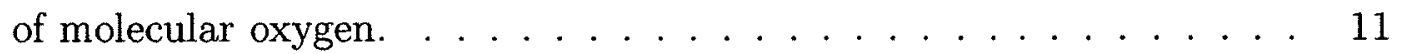

2.6 A plot of a theoretical SPDI spark current with the user interface parameters identified. . . . . . . . . . . . . . 22

2.7 Plots of voltage and current inside a combustion chamber of a running engine (TOP) and in the constant pressure conditions (BOTTOM). . 26

3.1 Geometric components of the cylinder; Bore $(b)$, half-stroke $(s / 2)$, crank angle $(\theta)$, piston position reference direction $(y) \ldots \ldots 28$

3.2 Geometry association for connecting rod, stroke length and crank angle. 29 
3.3 A plot of the engine resultant geometry parameters: piston position, piston speed and piston acceleration. . . . . . . . . . . 31

3.4 Plot of the pressure curve measured during the experiments with the appropriate strokes labelled (i.e. intake, compression, expansion, exhaust) . . . . . . . . . . . . . . . . . . . . 32

3.5 A sample P-V diagram identifying the areas for positive and negative work. . . . . . . . . . . . . . . . . . . . .

3.6 An example cylinder pressure versus its appropriate motored pressure. The deviation of the cylinder pressure from the motored pressure exists prior to ignition of the mixture. . . . . . . . . . . 40

4.1 The imaging setup for pressurized conditions. . . . . . . . . . . 43

4.2 This image corresponds to a single frame of the acquisition where breakdown is included showing the conduit of ionization as the magnetic field collapses the ionized particles into a single arc. . . . . . . .

4.3 This image corresponds to a single frame of the acquisition where the breakdown current was not included, this represents the oscillating voltage of the ignition device creating the plasma. . . . . . . . . .

4.4 This image corresponds to a single frame of the acquisition for the Inductive Discharge, it is apparent that the arc has been formed and the magnetic field has collapsed the ionized particles into the concentrated conduit.

4.5 From left to right, $\mathrm{BC}=50,75$ and 100. The rows (top to bottom) correspond to variable pressure (0 PSI, 50 PSI, 100 PSI and 150 PSI). 
4.6 Images of the first $260 \mu \mathrm{Sec}$ of discharge, the frames represent increasing pressure with constant SPDI spark breakdown current settings. The spark profile has the following settings: $\mathrm{BC}=100, \mathrm{SL}=40, \mathrm{IL}$ $=40, \mathrm{SP}=44, \mathrm{IP}=44 \ldots \ldots \ldots \ldots \ldots$

4.7 Photographs taken from 0 PSI to 90 PSI at 10 PSI increments, camera gate time of $260 \mu \mathrm{Sec}$ at a frame rate of $3800 \mathrm{~Hz}$, spark profile is; $\mathrm{BC}$ $=100, \mathrm{SL}=40, \mathrm{IL}=40, \mathrm{SP}=44, \mathrm{IP}=44$. All time labels are in microseconds.

4.8 Photographs taken from 100 PSI to 150 PSI at 10 PSI increments, camera gate time of $260 \mu \mathrm{Sec}$ at a frame rate of $3800 \mathrm{~Hz}$, spark profile is; $\mathrm{BC}=100, \mathrm{SL}=40, \mathrm{IL}=40, \mathrm{SP}=44, \mathrm{IP}=44$. All time labels are in microseconds. . . . . . . . . . . . . . . . . . 50

4.9 Discharges measured from 0 PSI to 50 PSI at 10 PSI increments, spark profile is; $\mathrm{BC}=100, \mathrm{SL}=40, \mathrm{IL}=40, \mathrm{SP}=44, \mathrm{IP}=44$. (See the top image in Figure 4.13 for an enhanced view of the red dashed section outlined in the 40PSI Figure here.) . . . . . . . . . . . . . 52

4.10 Discharges measured from 40 PSI to 70 PSI at 10 PSI increments, spark profile is $; \mathrm{BC}=100, \mathrm{SL}=40, \mathrm{IL}=40, \mathrm{SP}=44, \mathrm{IP}=44 \ldots$.

4.11 Discharges measured from 80 PSI to 110 PSI at 10 PSI increments, spark profile is; $\mathrm{BC}=100, \mathrm{SL}=40, \mathrm{IL}=40, \mathrm{SP}=44, \mathrm{IP}=44 \ldots .54$

4.12 Discharges measured from 120 PSI to 150 PSI at 10 PSI increments, spark profile is; $\mathrm{BC}=100, \mathrm{SL}=40, \mathrm{IL}=40, \mathrm{SP}=44, \mathrm{IP}=44$. (See the bottom image in Figure 4.13 for an enhanced view of the red dashed section outlined in the 140PSI Figure here.) . . . . . . . . 
4.13 Enhanced image of voltage and current for both modes of the discharge seen in Figure 4.9 and 4.11. Spark profile for both (top and bottom) is; $\mathrm{BC}=100, \mathrm{SL}=40, \mathrm{IL}=40, \mathrm{SP}=44, \mathrm{IP}=44 . \ldots 56$

5.1 The generator setup and its respective components labelled. . . . . . 59

5.2 The two encoder signals contributing to incremental changes of the data acquisition counters using X2 encoding methods. . . . . . . . . . 62

5.3 The two encoder signals contributing to incremental changes of the data acquisition counters using $\mathrm{X} 4$ encoding. . . . . . . . . . . . . 62

5.4 The effect of EMF noise overlapped on an encoder signal. . . . . . . . 63

5.5 Schematic of an encoder wired with pull-down resistors. . . . . . . . . 63

5.6 The installation location of the pressure sensor inside the cylinder head of the engine. . . . . . . . . . . . . . . 66

5.7 Adaptation of the ignition coils to monitor current through the spark plug. . . . . . . . . . . . . . . . . . . 6 67

5.8 Schematic of coil arrangements for both SPDI and Bosch Units. . . . 68

5.9 Setup for rotating the engine through the use of a PMG132 electric

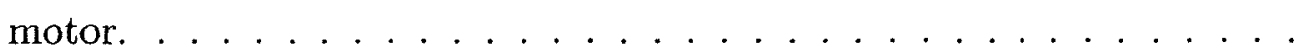

5.10 Motored pressure curves corresponding to throttle positions of $100 \%$, $50 \%$ and $0 \%$, all at 2870 RPM. . . . . . . . . . . . 71

5.11 TDC sensor voltage curves corresponding to three strokes of the engine. In the enhanced section, three arrows are drawn, the outers corresponding to the locations at which the switch in piston direction first occurs and therefore the midpoint would correspond to the location of TDC. . . . . . . . . . . . . . . . 
6.1 A plot of the filtered pressure overlapped over the non-filtered pressure curve. ...................... 80

6.2 Cylinder pressure versus crank angle for a single dataset trial which consists of five different sessions under the same engine conditions. The total number of curves is 1071 , where each session had approximately 250-260 individual cycles. . . . . . . . . . . . . . . 8

6.3 Mean, median and mode of cylinder pressure versus crank angle for a single dataset. . . . . . . . . . . . . . . . . . . . 84

6.4 Mean cylinder pressure and standard deviation, versus crank angle for a single dataset. . . . . . . . . . . . . . . . .

6.5 Histograms and normal probability plot for a single trials cylinder pressures corresponding to $180 \mathrm{DBTDC}$ crank angle location. . . . . . 86

6.6 Histograms and normal probability plot for a single trials cylinder pressures corresponding to 0 degrees crank angle location (TDC). . . 87

6.7 Histograms of crank angle corresponding to $\mathrm{MFB}=0.5$ for three different SPDI trials with the same spark profile and different engine loads, $355 \mathrm{~W}, 745 \mathrm{~W}$, and $1180 \mathrm{~W} \ldots \ldots \ldots \ldots 8$

6.8 Sample plot of MFB versus Crank Angle for a trial at $755 \mathrm{~W}$ engine load. The parameters used in the production of the histograms include $\mathrm{MFB}=0.5$ location, $\mathrm{MFB}$ rate at $\mathrm{MFB}=0.5$ location, and $\mathrm{MFB} \mathrm{EOC}$ corresponding to $\mathrm{MFB}=0.9$. . . . . . . . . . . . . . . . . 89

7.1 A plot of a typical spark voltage and current acquisition for three different SL settings $[0,20,40]$ starting at 20DBTDC. The current readings are inverse in polarity to coincide with the measurement method. 
7.2 Cylinder pressure versus crank angle for three spark durations at SPDI settings of: $\mathrm{BC}=50, \mathrm{SL}=\mathrm{IL}=[0,20,40], \mathrm{SP}=\mathrm{IP}=22$, and engine load of $60 \%$. (see Table 2.1 on page 23 for a description of the SPDI

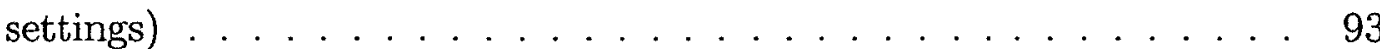

7.3 Cylinder pressure versus crank angle for three spark durations at SPDI settings of: $\mathrm{BC}=50, \mathrm{SL}=\mathrm{IL}=[0,20,40], \mathrm{SP}=\mathrm{IP}=33$, and engine load of $60 \%$. (see Table 2.1 on page 23 for a description of the SPDI

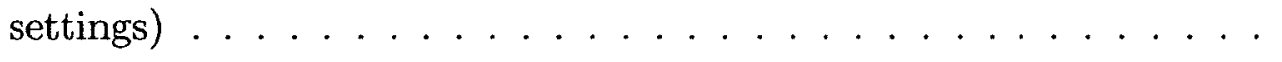

7.4 Cylinder pressure versus crank angle for three spark durations at SPDI settings of: $\mathrm{BC}=50, \mathrm{SL}=\mathrm{IL}=[0,20,40], \mathrm{SP}=\mathrm{IP}=44$, and engine load of $60 \%$. (see Table 2.1 on page 23 for a description of the SPDI settings) . . . . . . . . . . . . . . . . . . . .

7.5 Cylinder pressure versus crank angle for three spark durations at SPDI settings of: $\mathrm{BC}=50, \mathrm{SL}=\mathrm{IL}=0, \mathrm{SP}=\mathrm{IP}=[22,33,44]$, and engine load of $60 \%$. (see Table 2.1 on page 23 for a description of the SPDI

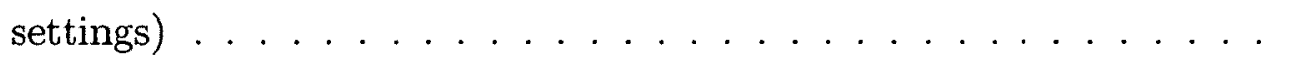

7.6 Cylinder pressure versus crank angle for three spark durations at SPDI settings of: $\mathrm{BC}=50, \mathrm{SL}=\mathrm{IL}=20, \mathrm{SP}=\mathrm{IP}=[22,33,44]$, and engine load of $60 \%$. (see Table 2.1 on page 23 for a description of the SPDI settings) ....................... 97

7.7 Cylinder pressure versus crank angle for three spark durations at SPDI settings of: $\mathrm{BC}=50, \mathrm{SL}=\mathrm{IL}=40, \mathrm{SP}=\mathrm{IP}=[22,33,44]$, and engine load of $60 \%$. (see Table 2.1 on page 23 for a description of the SPDI

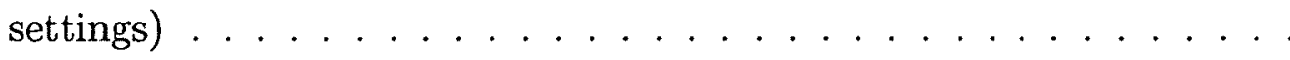


7.8 Cylinder pressure versus crank angle for three trials at an engine load of $60 \%$. (see Table 2.1 on page 23 for a description of the SPDI settings) 101

7.9 A plot of a typical spark voltage and current acquisition with the parameters identified. . . . . . . . . . . . . . . . . . 102

7.10 A histogram of crank angle values corresponding to $\mathrm{MFB}=0.5 \ldots \ldots$

7.11 A histogram of resulting MFB slope $\left[\frac{\mathrm{MFB}}{\mathrm{Deg}}\right]$ at $\mathrm{MFB}=0.5 \ldots \ldots . . .103$

7.12 A histogram of crank angles corresponding to EOC locations. . . . . 104

7.13 Plot of average standard deviation from their respective cylinder pressure means versus crank angle. . . . . . . . . . . . . 106

7.14 Plot of average percent standard deviation from their respective cylinder pressure means versus crank angle. . . . . . . . . . . . 107

7.15 Minimum $d_{0}$ for comparisons between each trial such that the hypothesis is not rejected. If this $d_{0}$ is below the 24.8 PSI threshold, the comparison results in statistically equivalent curves. If the $d_{0}$ is above the 24.8 PSI threshold, the comparison results in statistically NOT equivalent curves . . . . . . . . . . . . . . . 109

A.1 Sample Specifications for the Dantec Nanosense MKII High Speed CCD camera. . . . . . . . . . . . . . . . . . 118

A.2 The user interface for the ignition system and its adjustable parameters; Breakdown Current [mA], Ignition Duration [Deg], Ignition Power [W], Spark Duration [Deg], Spark Power [W] . . . . . . . . 119

B.1 Diagram of the circuits and ports/lines used for the engine setup and the National instruments data acquisition board array. . . . . . . 121

B.2 Schematic of coil arrangements for both SPDI and Bosch Units. . . . 122 
B.3 Schematic of power supply unit made to regulate $12 \mathrm{~V}$ down to $5 \mathrm{~V}$ and $10 \mathrm{~V}$

B.4 Image of physical coil used in the experiments. . . . . . . . . . . 123

C.1 Flow chart of data analysis and addition to the database. . . . . . . 125

C.2 An image of the front panel used in the MATLAB database evaluation program . . . . . . . . . . . . . . . . 126

C.3 A plot of the residuals from subtracting the filtered data from the non-filtered data. $\ldots \ldots \ldots \ldots \ldots \ldots \ldots \ldots$

C.4 Histograms and normal probability plot for a single trials cylinder pressures corresponding to 180 degrees crank angle location. . . . . . 128

C.5 A plot of the mean, median and mode for a sample pressure trace acquisition inside the combustion chamber of a single cylinder engine.

D.1 Cylinder pressure versus volume for three spark durations at SPDI settings of: $\mathrm{BC}=50, \mathrm{SL}=\mathrm{IL}=[0,20,40], \mathrm{SP}=\mathrm{IP}=22$, and engine load of $60 \%$. (see Table 2.1 on page 23 for a description of the SPDI settings)

D.2 Cylinder pressure versus volume for three spark durations at SPDI settings of: $\mathrm{BC}=50, \mathrm{SL}=\mathrm{IL}=[0,20,40], \mathrm{SP}=\mathrm{IP}=33$, and engine load of $60 \%$. (see Table 2.1 on page 23 for a description of the SPDI settings)

D.3 Cylinder pressure versus volume for three spark durations at SPDI settings of: $\mathrm{BC}=50, \mathrm{SL}=\mathrm{IL}=[0,20,40], \mathrm{SP}=\mathrm{IP}=44$, and engine load of $60 \%$. (see Table 2.1 on page 23 for a description of the SPDI settings) 
D.4 Cylinder pressure versus crank angle for three spark durations at SPDI settings of: $\mathrm{BC}=50, \mathrm{SL}=\mathrm{IL}=0, \mathrm{SP}=\mathrm{IP}=[22,33,44]$, and engine load of $60 \%$. (see Table 2.1 on page 23 for a description of the SPDI

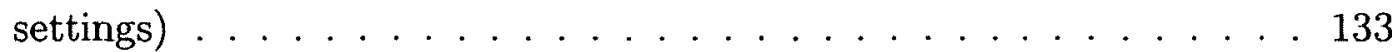

D.5 Cylinder pressure versus crank angle for three spark durations at SPDI settings of: $\mathrm{BC}=50, \mathrm{SL}=\mathrm{IL}=20, \mathrm{SP}=\mathrm{IP}=[22,33,44]$, and engine load of $60 \%$. (see Table 2.1 on page 23 for a description of the SPDI settings $\ldots \ldots \ldots \ldots \ldots \ldots \ldots \ldots$

D.6 Cylinder pressure versus crank angle for three spark durations at SPDI settings of: $\mathrm{BC}=50, \mathrm{SL}=\mathrm{IL}=40, \mathrm{SP}=\mathrm{IP}=[22,33,44]$, and engine load of $60 \%$. (see Table 2.1 on page 23 for a description of the SPDI settings $\ldots \ldots \ldots \ldots \ldots \ldots \ldots \ldots \ldots$

D.7 Cylinder pressure versus crank angle for three trials, two SPDI and one ID at an engine load of $60 \%$. (see Table 2.1 on page 23 for a description of the SPDI settings) $\ldots \ldots \ldots \ldots \ldots$ 


\section{List of Acronyms}

\begin{tabular}{ll}
\hline Acronyms & Definition \\
\hline \hline BDC & Bottom Dead Centre \\
DATDC & Degrees After Top Dead Centre \\
DBTDC & Degrees Before Top Dead Centre \\
EGT & Exhaust Gas Temperature \\
EOC & End of Combustion \\
FWHM & Full-Width Half-Maximum \\
GUI & Graphical User Interface \\
ID & Inductive Discharge \\
IT & Intake Temperature \\
MFB & Mass Fraction Burn \\
PDF & Probability Density Function
\end{tabular}

xxii 


$\begin{array}{ll}\text { PWM } & \text { Pulse-Width Modulation } \\ \text { PWT } & \text { Pulse-Width Timing } \\ \text { RPM } & \text { Revolutions Per Minute } \\ \text { SPDI } & \text { Spatial Plasma Discharge Ignition } \\ \text { TDC } & \text { Top Dead Centre } \\ \text { TPS } & \text { Throttle Position Center }\end{array}$

xxiii 


\section{List of Symbols}

\begin{tabular}{ll} 
Symbols & Definition \\
\hline \hline$\sigma$ & Standard Deviation \\
$\mathrm{V}$ & Voltage \\
$\mathrm{I}$ & Current \\
$\mathrm{R}$ & Resistance \\
$V$ & Volume \\
$V_{\mathrm{CL}}$ & Clearance Volume \\
$V_{\mathrm{D}}$ & Displacement Volume \\
$V_{\mathrm{cyl}}$ & Cylinder Volume \\
$V_{\mathrm{BDC}}$ & Cylinder Volume at Piston Position of BDC \\
$L$ & Connecting Rod Length \\
$s$ & Stroke Length
\end{tabular}

xxiv 


$\begin{array}{ll}b & \text { Cylinder Bore Diameter } \\ v_{\mathrm{p}} & \text { Piston Velocity } \\ a_{\mathrm{p}} & \text { Piston Acceleration } \\ \omega & \text { Photon Wave Length } \\ \phi & \text { Fuel to Air Equivalence Ratio } \\ W & \text { Work } \\ P & \text { Pressure } \\ \theta & \end{array}$




\section{Chapter 1}

\section{Introduction}

The extensive use of internal combustion engines in transportation and other applications has increased the demand for fuel efficiency and lower emission pollutant. Over the past few decades many techniques such as optimizing air/fuel turbulence, mixture homogeneity, cylinder and piston design, fuel constituent modifications, as well as ignition system research have been the focus in efforts to increase fuel efficiency. However, one of the most recent areas of interest in combustion optimization is determining the role that low temperature plasmas or current pulse-width modulated (PWM) systems can contribute to the combustion process. New innovations and techniques of flame monitoring have brought the previously unknown chemical kinetics of combustion into perspective. Recently, the effects of current pulse-width modulated plasmas on combustion to alter the chemical reaction pathways in the combustion process have been investigated for use in internal combustion engines. 


\subsection{Outline}

This thesis will begin with background information and relevant literature pertaining to plasma kinetics, combustion, and plasma-assisted combustion. Then, it will continue through a description of the specific experimental apparatus used in this study, the post-processing techniques and finally a discussion of the results.

\subsubsection{Background Information and Literature Review}

The second and third chapter of this thesis will discuss background information of plasma chemistry and internal combustion engine basics. A brief discussion of relevant literature for plasma ignition devices in spark ignition engines will be presented. The trends and conclusions in most papers argue that the combustion process is accelerated when plasma ignition devices are used in place of traditional ignition devices. Specifically, higher burn rates in mass fraction burn analyses and lower ignition delay times are observed. In addition to the experimental results, current research in modelling plasma ignition devices in combustion is also underway, and the thesis will also review these papers and their results.

\subsubsection{Imaging the Spark Propagation}

Chapter four will present our results pertaining to the imaging of the spark propagation and plasma formation from the ignition device. An overview of the luminous and non-luminous states of the plasma will also be presented. The experimental investigation made use of two apparatuses; one at ambient pressure and temperature, the other at a pressurized state and ambient temperature. The results gathered 
from optically imaging the spark propagation as well as monitoring the voltage and current curves will be compared to other research in this subject from the literature.

\subsubsection{Experimental Methods}

The primary topic of this thesis is a set of experiments to measure pressure rise rates inside the combustion chamber, described in Chapter 5. The experiment employed a single cylinder engine outfitted with a pressure sensor to measure the pressure rise rates inside the combustion chamber and an angular encoder for crank angle measurements. To minimize deviations in the cyclic repeatability for ignition timing, the ignition triggering system developed for this engine used the LabVIEW ${ }^{1}$ software and National Instruments hardware configuration boards. The ignition systems used in the experiments include a BOSCH inductive discharge (ID) unit and a Spatial Plasma Discharge Ignition (SPDI) device that provides an initial discharge arc followed by the formation of a plasma. Other techniques for monitoring the engine performance (for example exhaust gas emissions, torque output, horsepower output and fuel efficiency), are left for future work to keep this current investigation relatively simple and focussed. Of course, an understanding of SPDI's effects on all these parameters are eventually of interest.

Chapter 6 presents our developments of the post-processing techniques used to analyze the data that were collected from the engine experiment. Three programs were constructed. The first used the LabVIEW software to control the engine, the second used MATLAB to analyze the signals, and the third optimized the first two

\footnotetext{
${ }^{1}$ LabVIEW is a graphical programming utility typically used for data acquisition and system control. The data flow interface allows for complex pieces of code to be programmed easily and efficiently.
} 
programs by making a real-time data acquisition and analysis program in LabVIEW.

\subsubsection{Results and Discussions}

Chapter 7 presents the results and discussions of the experiment by explaining the variations in the plasma power setting and the corresponding effects on the pressure curves and mass fraction burn rates. The ignition delay parameters of the SPDI ignition device versus that of the inductive discharge are analyzed and conclusions are made. 


\section{Chapter 2}

\section{Background Information and Relevant Literature}

\subsection{Plasma Ionization and Molecular Interactions}

The term plasma, although commonly used today in plasma televisions, was conceived in 1928 by Irving Langmuir [1]. It is now known as the fourth state of matter along with the first three; solid, liquid and gas. In general, a plasma is a collection of ionized particles. The molecular interactions, molecular energy states and the types of plasma discharges will be discussed further in the following sections. The present research uses plasma-assisted ignition to affect the combustion process, therefore topics related to this will be discussed as well.

\subsubsection{General Atom or Molecule Excitation}

Introducing one of the most commonly known atomic models today, Neils Bohr wrote about the spherical approximation of an atom with its electrons circling about 
its nucleus. He performed vacuum tube experiments that sought to identify the wavelength of the light emitted by exciting elements through electric discharges [2]. His model, Figure 2.1, shows the nucleus at the centre and some figurative shells surrounding it. Each shell, can be decomposed into individual orbitals, for example, the $n=1$ shell corresponds to the ' 1 s' orbital, while the $n=2$ shell corresponds to the ' $2 \mathrm{~s}$ ' and ' $2 \mathrm{p}$ ' orbitals, the $\mathrm{n}=3$ shell corresponds to the ' $3 \mathrm{~s}$ ', ' $3 \mathrm{p}$ ', and ' $3 \mathrm{~d}$ ' orbitals, and so on. In this notation the $\mathrm{n}=1,2,3$, etc. represent the shell level and the $\mathrm{s}, \mathrm{p}$, $\mathrm{d}$, represents the orbitals. Physicists today have a more sophisticated understanding of these orbitals, but the Bohr idea remains useful as a conceptual tool. It shows us how the electrons can only occupy certain well-defined energy states.

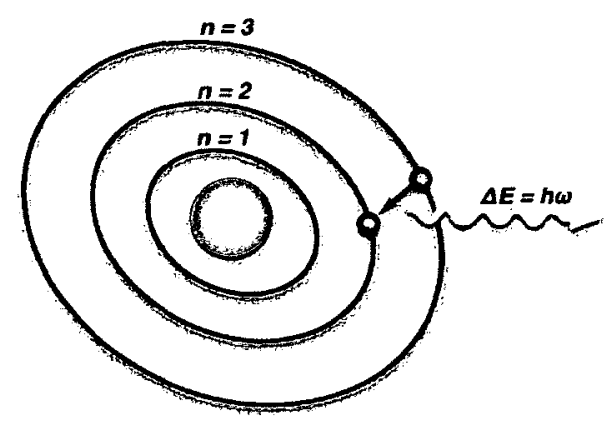

Figure 2.1: Bohr model of an atom with its nucleus at the centre and an electron dropping from its outer most orbital to the second orbital followed by the emission of a photon.

\section{1s - Orbital and 2s - Orbital}

These represent the first two orbital levels, consisting of two concentric spherical volumes around the nucleus in which the first pair of electrons will be found most commonly (inside the ' $1 \mathrm{~s}$ ' volume) and the second pair of electrons will be found most commonly (inside the ' $2 \mathrm{~s}$ ' volume). Once these shells have reached max capacity (2 
electrons each) the excess electrons begin to fill the ' $2 p$ ' orbitals.

\section{2p - Orbitals}

The $2 \mathrm{p}$ orbitals are a group of volumes in which up to 6 electrons can be dispersed within specific sharing criterion. The orbital sections include $p_{x}, p_{y}$, and $p_{z}$, which are different orientations of the same shape (Figure 2.2) and have a maximum capacity of 2 electrons each.

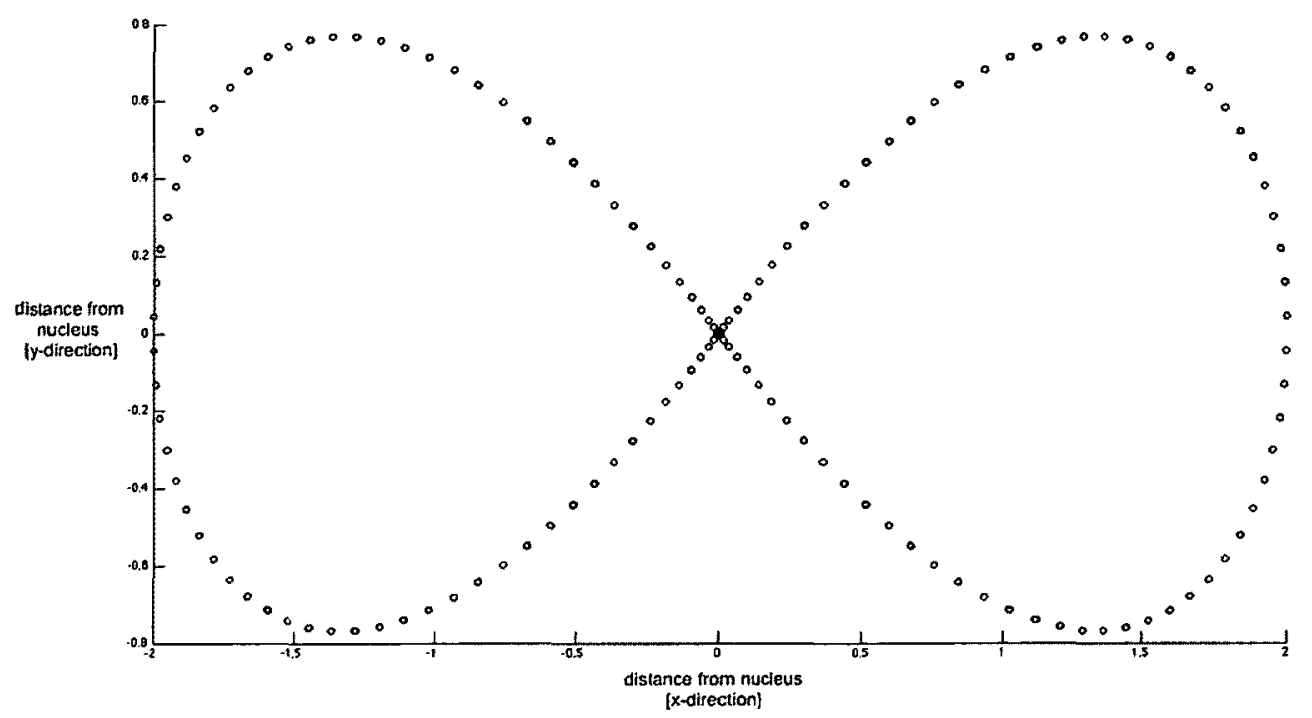

Figure 2.2: A two dimensional representation of the volume limit in which the electrons can be found for a single $2 p$ type orbital.

Beyond the ' $2 \mathrm{p}$ ' orbitals, there exists many more shells and subsequently more orbital shapes determined by the number and letter of its notation (i.e. $3 \mathrm{~s}, 3 \mathrm{p}, 3 \mathrm{~d}$, $4 \mathrm{~s}, 4 \mathrm{p} 4 \mathrm{~d}$, etc..).

In atoms and molecules there exist three types of excitation modes; vibrational, translational and electronic, each of which renders the molecule into a metastable 
state $^{1}$. Through electron excitation, the electron-electron interaction forces and magnetic coupling forces within a single orbital can cause two atoms to have different levels of excitation without ionization. This is termed electronic excitation. ${ }^{2,3}$ The common notation for a general molecule at its lowest energy (ground state) is identified by $\mathrm{M}$. For positively ionized molecules it is $\mathrm{M}^{+}$, and for negatively ionized molecules, $\mathrm{M}^{-}$, for the case of electronically excited molecules it is $\mathrm{M}^{*}$. This excited molecule notation $\left(\mathrm{M}^{*}\right)$ can represent any degree of excitation that the molecule can attain. As was previously mentioned, electron configurations can control the degree of this excitation energy and can therefore be sub-categorized using more specific symbols.

The basis of electronic excitation is the configuration of the atom's or molecule's electrons. The simplest case is when an electron receives enough energy (from an outside source) that it moves from one shell level to a higher shell level (i.e. between $\mathrm{n}=1,2,3$, etc.). Similarly, a molecule can relax, resulting in the electron moving from the outer shell back down to the ground state shell of the molecule, as depicted in Figure 2.1. In this reverse process an emission of a photon occurs with energy equal to the difference in potential caused from the electron transition. This photon's energy is equal to $h \omega$, or Planck's constant $\left(h=6.626 e^{-34}[J \cdot s][4]\right)$ multiplied by the frequency $(\omega)$ of the photon. The photon frequency can be measured, and therefore it allows us to determine the excitation levels of the atoms since each atom has

\footnotetext{
${ }^{1} \mathrm{~A}$ metastable state is defined by Oxford Dictionaries as a state that is "theoretically unstable but so long-lived as to be stable for practical purposes". [3] For the case of this thesis it refers to a particle that will remain at an excited state until a change in its energy occurs.

${ }^{2}$ Vibrational excitation is a type of energy in which the particle moves side to side with a frequency and amplitude corresponding to the amount of vibrational excitation.

${ }^{3}$ Translational excitation is a type of energy in which the particle moves with a momentum in a single direction until affected by another outside source.
} 
prescribed differences in potential energy between its shell levels.

For a photon emission to occur, it is not required that an electron travel from one shell level to another, a reconfiguration of the electron location and spin direction can also lead to this result.

As a physical example, excited molecular oxygen $\left(\mathrm{O}_{2}\right)$ can take the form of three states: triplet oxygen, also known as the ground state $\left({ }^{3} \Sigma_{g}^{-}\right)$, the lowest energy singlet state $\left({ }^{1} \Delta_{g}\right)$ and its second excited singlet state $\left({ }^{1} \Sigma_{g}^{+}\right)$. According to the molecular orbital (MO) diagram (for readers familiar with MO diagrams please refer to Figure $2.3,2.4,2.5)$ these molecules only differ in electron spin and orbital occupation, however they represent the ground state and excited states of a single oxygen molecule. Fundamentally the two singlet excited states are identified as; $O_{2}\left(a^{1} \Delta_{g}\right)$ and $\mathrm{O}_{2}\left(b^{1} \Sigma_{g}^{+}\right)$, however in this thesis, and in literature, they are combined and represented in a general form, such as $\mathrm{O}_{2}^{*}$, or for the case of general equations, $\mathrm{A}^{*}$, with A representing an arbitrary molecule. 


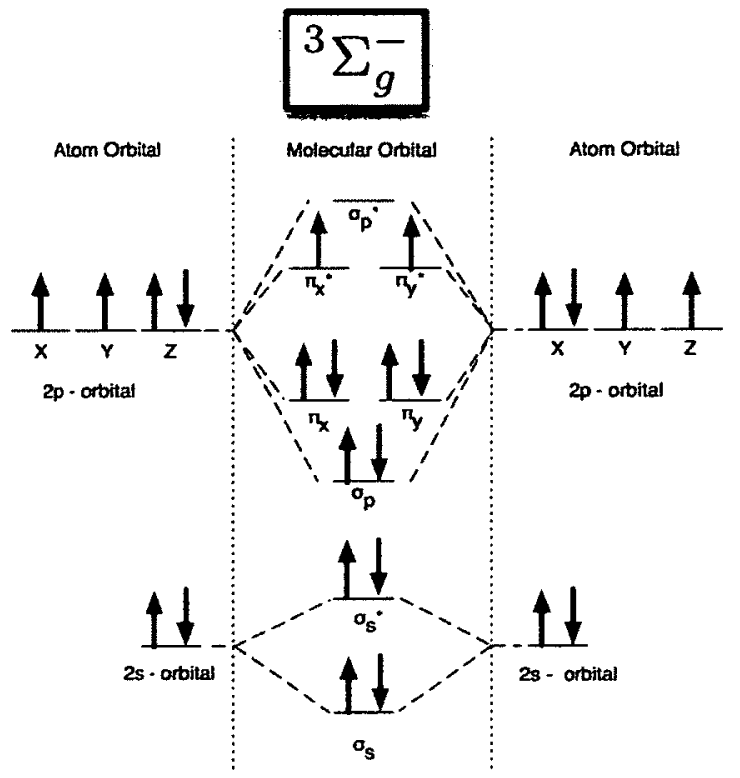

Figure 2.3: Molecular Orbital (MO) diagram for the triplet oxygen molecular state.

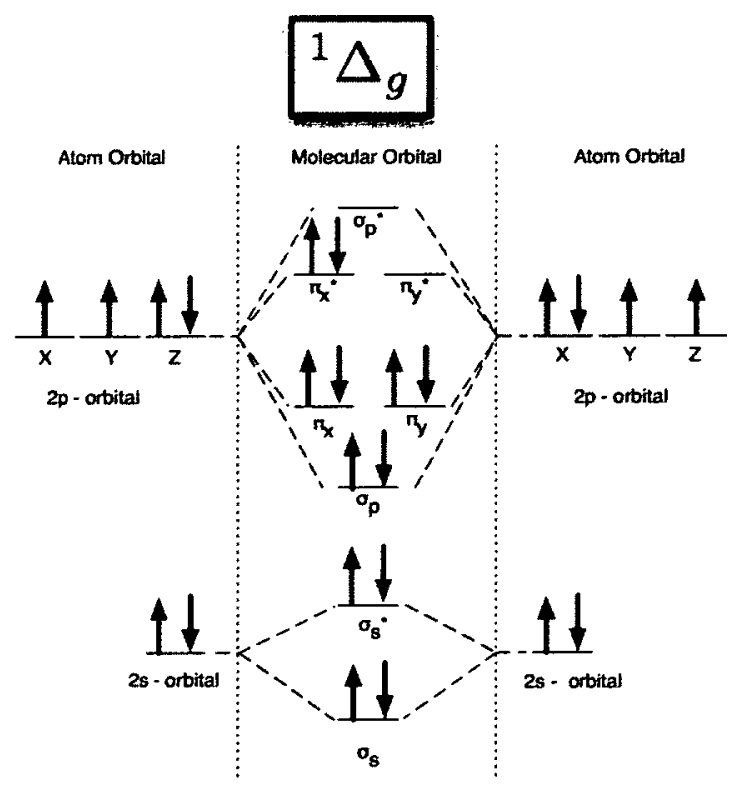

Figure 2.4: Molecular Orbital (MO) diagram for the lowest energy singlet state of molecular oxygen. 


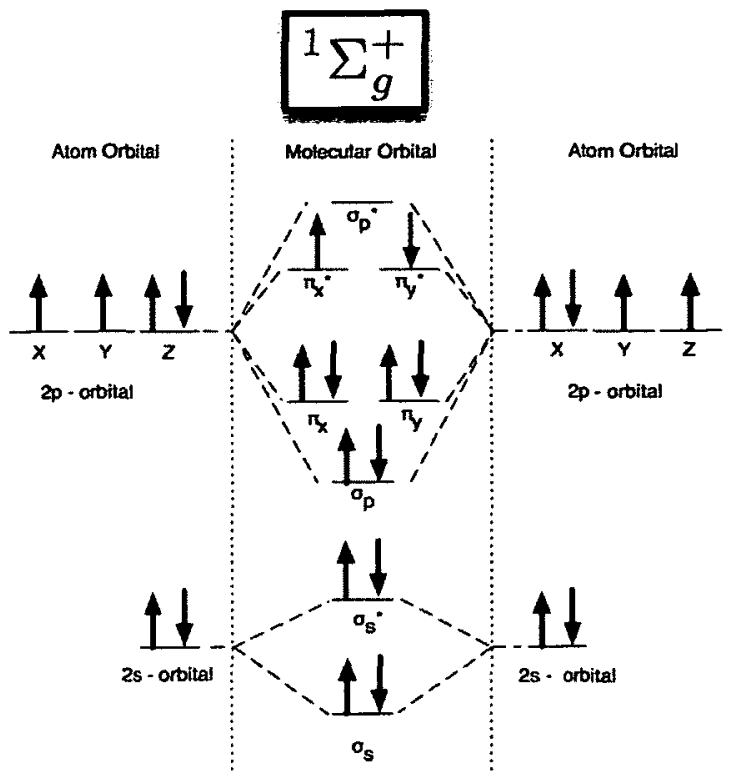

Figure 2.5: Molecular Orbital (MO) diagram for the second energy singlet state of molecular oxygen.

Unlike the case shown in Figure 2.1 where the electron travels from the 3rd level shell to the 2nd level shell, the excited states of $\mathrm{O}_{2}$ consist of reorientation between the electrons within the 2nd level shell due to electron-electron interactions and magnetic coupling. Two examples of such process as listed by Jockusch et al.[5] include:

$$
\left[{ }^{1} \Delta_{g}(\nu=0)\right] \rightarrow\left[{ }^{3} \Sigma_{g}^{-}(\nu=0)\right]+h \omega[1270 n m]
$$

and

$$
\left[{ }^{1} \Sigma_{g}^{+}(\nu=0)\right] \rightarrow\left[{ }^{3} \Sigma_{g}^{-}(\nu=0)\right]+h \omega[765 n m]
$$


where each results in an emission of photons whose wavelength are $1270 \mathrm{~nm}$ and $765 \mathrm{~nm}$ respectively.

In some cases discussed in this thesis, only some excited species are capable of exhibiting the necessary traits to conduct the reactions shown. However, with the caveat that the $\mathrm{A}^{*}$ symbol represents multiple excitation levels, the following sections will discuss the interactions among ground state molecules (A), excited molecules $\left(\mathrm{A}^{*}\right)$ and ionized molecules $\left(\mathrm{A}^{+}\right.$and $\left.\mathrm{A}^{-}\right)$.

In addition, the terms electron temperature $\left(T_{e}\right)$, and atom temperature $\left(T_{0}\right)$ will be used. For the heavy particles in a system, their temperature is calculated by

$$
T_{0}=\frac{2<E_{0}>}{3 k}
$$

where $k$ is the Boltzmann constant $=1.38065 \times 10^{-23} \mathrm{~J} \cdot \mathrm{K}^{-1}[4]$ and $\left\langle E_{0}\right\rangle$ is time-averaged kinetic energy of the particles. This is the usual "temperature" that we encounter in non-plasma physics and everyday life. In a plasma, the free electrons in the system (assuming a Maxwellian distribution) have a temperature that is calculated by

$$
T_{e}=\frac{2<E_{e}>}{3 k}
$$

where $\left\langle E_{e}\right\rangle$ is the time-averaged kinetic energy of the electrons. Because electrons are so much lighter than the heavy particles in a gas, the energy transfer between electrons and heavy particles is very poor. As such $T_{0}$ and $T_{e}$ can be very different in a plasma [6]. 
Note: Sections 2.1.2 to 2.1.4 follow closely the presentation given by A.Fridman [1].

\subsubsection{Molecular Interaction Methods}

Molecular collisions can be categorized into three main collision types: elastic collisions, inelastic collisions and superelastic collisions. Each of these has a different effect on the atoms as they scatter after colliding. For example, elastic collisions do not convert kinetic energy into other forms. The net kinetic energy of all atoms involved in an elastic collision remain the same, while inelastic collisions can convert kinetic energy into internal energies, therefore reducing the net kinetic energy of all bodies involved in the collision. Meanwhile superelastic collisions refer to the reverse process of inelastic collisions, where the internal energy is transferred into kinetic energy (this process is common for plasma electrons in plasma interactions [1]). These energy collision processes define molecular interaction for neutral species, ionized species, and electrons.

During inelastic collisions, ionization can occur. The ionization process consists of a neutral particle becoming electrically charged through the removal or addition of an electron, as a neutral molecule consists of having an equivalent number of electrons and protons such that there is no electric charge. One may ask: how does a molecule lose an electron if its lowest energy state is that in which it is chemically neutral? There are five main ionization processes that can occur;

- Direct ionization by electron impact: This occurs when an electron has enough energy to ionize an atom in one collision. It is claimed by many researchers ([7], [8] and [9]) that due to the presence of high electric fields in plasmas, this 
mechanism is important in the creation of low temperature plasma ions and also in the emission of excited species. The process can be depicted as follows;

$$
\mathrm{e}+\mathrm{AB} \rightarrow \mathrm{AB}^{+}+\mathrm{e}+\mathrm{e} .
$$

If the electron energy is much higher than the ionization energy of the diatomic molecule, the result could be a dissociation along with an electron emission [1], or

$$
\mathrm{e}+\mathrm{AB} \rightarrow \mathrm{A}+\mathrm{B}^{+}+\mathrm{e}+\mathrm{e}
$$

- Stepwise ionization by electron impact: This occurs when an electron bombards an already excited molecule. This process is similar to that of direct ionization although it requires an electron to collide with a neutral species in an inelastic manner, thereby giving the atom internal energy. Then another electron of low energy collides with the already excited atom, ionizing it and removing an electron: [1]

$$
\begin{gathered}
\mathrm{e}+\mathrm{AB} \rightarrow \mathrm{AB}^{*}+\mathrm{e}, \\
\mathrm{e}+\mathrm{AB}^{*} \rightarrow \mathrm{AB}^{+}+\mathrm{e}+\mathrm{e} .
\end{gathered}
$$

- Ionization by collision of heavy particles: Although this process does occur, it is not frequent. The transfer of kinetic energy from a large molecule or atom to the electrons of another molecule does not occur as frequently as the energy 
transfer between two electrons.[1]

- Photo-ionization: This process occurs when a photon (hw) interacts with a neutral species to remove one of its electrons which leads to a positive ion and an electron scattering, as

$$
\mathrm{h} \omega+\mathrm{A} \rightarrow \mathrm{A}^{+}+\mathrm{e}
$$

where the photon is at a given wavelength. For the photo-ionization process to occur, it is common that the wavelength be less than $1000 \AA$. [1]

- Surface ionization: This occurs when an electron is removed from a surface due to ion-surface, photon-surface or molecule-surface interactions. The discussion on surface ionization will continue in section 2.1.4.

The processes of collisions and ionizations can yield positive or negative ions depending on the interacting molecules and their surroundings. The topic of positive ion interactions is discussed in the next section.

\subsubsection{Positive Ion Chemical Reactions and Recombination}

Most ionization reactions that yield positive ions typically do not have any activation energy. This means that the chemical interactions of the positively ionized molecules do not depend on their internal energies, they are at liberty to chemically react with each other without an activation energy barrier [1]. This, in conjunction with the exothermic reaction of electron recombination, is a very important concept in plasma chemistry. 
The first type of recombination is dissociative ion-electron recombination seen by the following equation;

$$
\mathrm{e}+\mathrm{AB}^{+} \rightarrow \mathrm{AB}^{*} \rightarrow \mathrm{A}+\mathrm{B}^{*}
$$

It is notable that the process does not occur as a single stage, the electron-ion interaction leads firstly to a metastable electronically excited molecule $\left(A B^{*}\right)$, followed by the dissociation resulting in the formation of a neutral $(A)$ and an electronically excited molecule or atom $\left(B^{*}\right)$. This process is very fast, since it has no activation energy it is not dependent on electron temperature $\left(T_{e}\right)$ or atom temperature $\left(T_{0}\right)$ [1]. If the pressure is high enough, a process called an ion conversion reaction happens prior to the dissociative ion-electron recombination. This process can be seen by the following reaction of Xenon [1];

$$
\mathrm{Xe}^{+}+\mathrm{Xe}+\mathrm{Xe} \rightarrow \mathrm{Xe}_{2}^{+}+\mathrm{Xe}
$$

Since this process readily happens at high pressures, the ion-electron recombination process can furthermore be performed on these newly activated diatomic molecules ${ }^{4}$. This process is important because the creation of polyatomics ${ }^{5}$ of nitrogen and oxygen at high pressures have very fast reaction rates and therefore recombine with a significant number of electrons in the system [1]. This can be particularly important when associating the plasma with such processes as combustion, where the amount of reactive oxygen and nitrogen is important for the reaction rates of the entire process.

The second ion-electron recombination process is the three-body and radiative

${ }^{4}$ Diatomic molecules are molecules consisting of only two atoms.

${ }^{5}$ Polyatomic molecules are molecules consisting of more than two atoms. 
electron-ion recombination process. In the state of quasi-equilibrium plasmas, the atoms recombine with electrons to form electronically activated atoms as seen by [1]

$$
\mathrm{e}+\mathrm{e}+\mathrm{A}^{+} \rightarrow \mathrm{A}^{*}+\mathrm{e}
$$

The third body in this reaction process includes the electron that gains the remaining kinetic energy from the event, and is therefore potentially a good candidate for direct electron impact ionization of another molecule or atom. Radiative ion-electron recombination is a relatively slow process compared to dissociative ion-electron recombination processes, due to the emission of a photon during the electron-ion interaction time [1]. This process can be seen by

$$
\mathrm{e}+\mathrm{A}^{+} \rightarrow \mathrm{A}^{*} \rightarrow \mathrm{A}+\mathrm{h} \omega
$$

which includes the metastable, electronically-excited state of the atom $\left(A^{*}\right)$.

\subsubsection{Electron Emissions in Plasma States and Electric Fields}

During plasma conditions, there are almost always electron currents inducing electric fields. In examples such as in a combustion chamber or across two electrodes, the electric fields have the propensity to create electron emissions from metal surfaces. The processes discussed in this section focus on thermionic emissions, field emissions and secondary electron emissions. It should be noted that the following processes induce electron velocities that can be used to ionize species within the local area 
through electron-atom interactions [1].

In thermal plasmas where the temperatures of the atoms is greater than the temperature of the electrons $\left(T_{0}>T_{e}\right)$, the atoms within the metal surface eject electrons into the surroundings, this process is called a thermionic emission. In cases where the emission is located near the cathode, the field created from the cathode to anode has enough force to expel the electrons away from the surface. This can further allow electrons to be emitted from the metal. A quantity known as the work function determines the amount of energy required for an electron to be extracted from a metal surface, for example it requires $4.54 \mathrm{eV}[1]$ to extract an electron from a tungsten surface. Under conditions of high electric fields, such work functions can be minimized, allowing a large amount of electrons to be ejected. This is known as the Schottky Effect.

The field emission of electrons, where electric fields are near $0.33 \times 10^{6} \mathrm{~V} / \mathrm{cm}[1]$, are able to extract electrons through the Schottky effect and also through a phenomenon called tunnelling. This phenomenon is responsible for allowing electrons, of a given kinetic energy, to pass through a potential energy barrier of a greater value at the surface of the metal. The high electric fields allow for reduction in the work function and ease of quantum tunnelling as well as thermoionic emissions, the result is a higher propensity for electron emission versus simply thermoionic processes [1].

The final mode of electron emission is called secondary electron emissions, similarly to the function of a scanning electron microscope where the metal surface undergoes bombardment from electrons, in secondary electron emissions, the surface undergoes particle bombardment resulting in the release of surface electrons. There 
are different types of particles that can induce secondary electron emission, examples of such particles are ions, metastable atoms, and electrons. As well, photon bombardment will result in secondary electron emission.

\subsection{Relevant Literature in Plasma Discharge Ig- nition Systems}

Research into plasma effects on combustion has become more common since the inception of high power lasers and high frequency pulse width modulation systems. Some examples of research groups investigating plasma ignition devices include; Lou et al. [10], Cathey et al. [11] or A.Y. Starikovskii [12].

Research groups such as Singleton et al. [7], have proven that the use of nonthermal transient plasma can decrease ignition delays in combustion through use of a combustion reactor experiment. They and others, [13-17] have made use of nanosecond pulsed power ignition systems to alter the chemistry of the reactants before combustion and create new chemistry branches that would otherwise not exist. For example, Wu et al. [18] made use of a methane-air flow experiment and postulate that the molecular hydroxides become electronically excited prior to combustion, which lead to lengthened oxidation chains by combustible mixtures at temperatures as low as $400-800 \mathrm{~K}$. This is lower than the ignition threshold of the mixtures in current kinetic models.

A.Y.Starikovskii [12] postulates that the molecular $N_{2}$ becomes electronically excited and alters the chemistry prior to kernel formation and flame propagation, where the effects of electron-excited particles play a dominant role. They've concluded that 
the flame propagation velocity achieved by nanosecond pulsed discharge excitation is twice that of the non-excited flame. Stable flames where $\phi=0.55$ for plasma-assisted combustion conditions achieve the same flame velocities as those with $\phi=1.1$, without plasma excitation.

An example of combustion analysis and resulting constituents is the work presented by Lou et al. [10], who used Fourier Transform (FT) absorption spectra to determine the concentration of $\mathrm{H}_{2} \mathrm{O}, \mathrm{CH}_{4}, \mathrm{CO}_{2}$ and $\mathrm{CO}$ downstream of a $40 \mathrm{kHz}$ nanosecond pulsed plasma discharge, in a hydrocarbon-air mixture flow reactor. They showed that for the "plasma on" condition, the FT absorption spectra identified higher concentrations of $\mathrm{H}_{2} \mathrm{O}, \mathrm{CO}_{2}$ and $\mathrm{CO}$ compared to that of the "plasma off" condition.

The research groups identified here make use of voltage pulses in the 10ns region by Wu et al. [18] or up to 40ns by Starikovskii [12]. Comparing this to the device used in this research ( $\mu$ s range) it is evident that the full-width half-maximum (FWHM) of these ignition devices are significantly faster. It is notable that Shiraishi et al. [19] postulated that the higher electric fields resulting from shorter pulse durations of equivalent energy will provide better ignitability of the mixture. Therefore, with the larger pulse width used in our research, due to the devices limits, it is possible that the effects seen in the literature will not be as evident in our results.

Recently, using numerical models, research groups are investigating the effects of flame propagation speeds and ignition delay times due to ignition from plasma devices. Such groups include Bourig et al. [20], who investigated the effects of electronically excited species on flame propagation of $\mathrm{H}_{2} \mathrm{O}_{2}$ flames, and Do [21] who made 
use of the CANTERA combustion simulation software by simulating the enhancement in concentration of hydroxide molecules in a reactor, to research the effects of plasma-assistance on combustion in supersonic flows.

The conclusions drawn from the research of these groups suggest that the ignition delay of combustion, with plasma assistance, is less than that when no plasma is used. The maximum cylinder pressure is also higher for plasma ignition devices compared to that of regular inductive or capacitive discharge units. This thesis will compare these results from the literature to those of our research group.

\subsection{The Plasma Settings and Parameters}

The plasma ignition device used in this research offers parameter variations controlled by the user interface in Figure A.2. These parameters control the duration and energy of the spark. A sample spark profile with some of its components labelled is seen in Figure 2.6. The Spark Power and Ignition Power from Table 2.1 cannot be depicted directly in this figure, however they are represented by the two amplitudes of the oscillating current (Ignition Power - controls pulse widths during the Ignition Duration, Spark Power - controls the pulse widths during the Plasma Duration). 


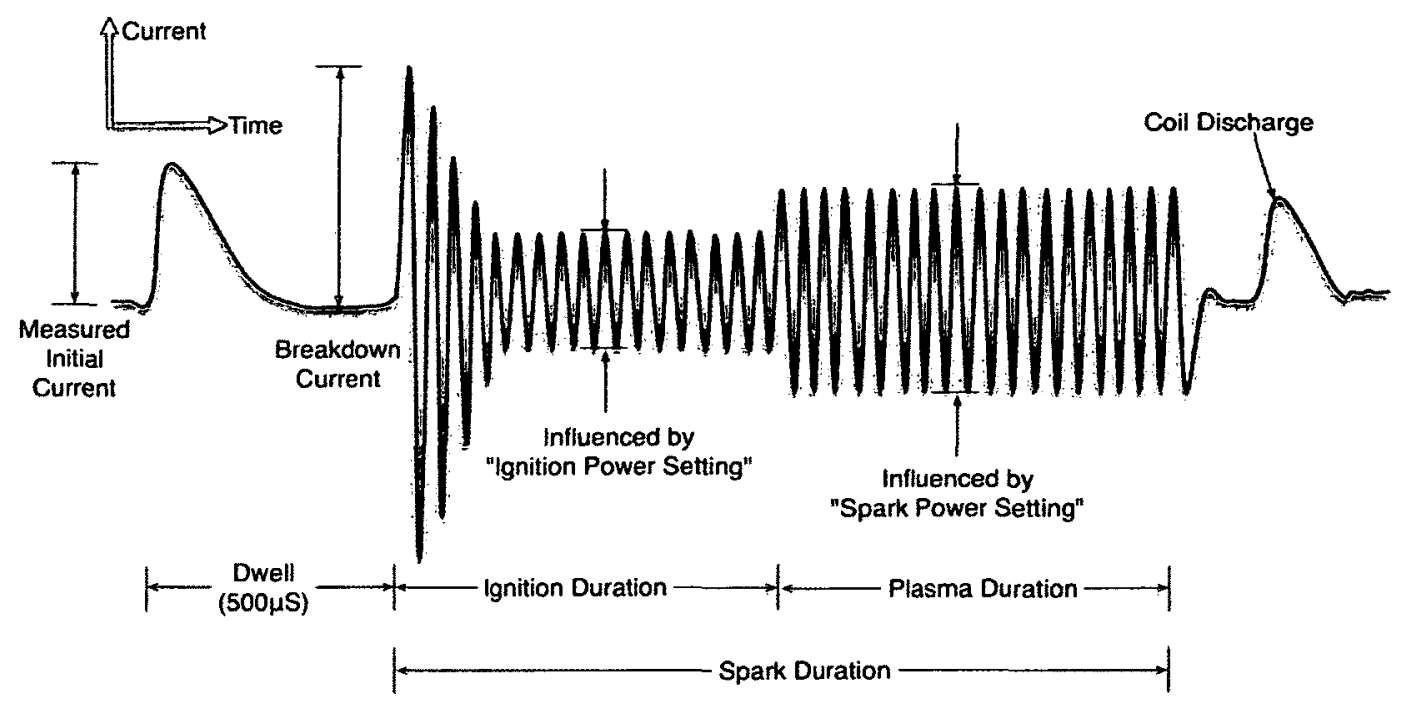

Figure 2.6: A plot of a theoretical SPDI spark current with the user interface parameters identified.

A brief description of each parameter and its resulting physical influence on the ignition device can be seen in Table 2.1.

Through the configuration software (Figure A.2) made available by the manufacturer, it is possible to set these parameters. However, since real-time adjustment of parameters is not available, the parameters listed in Table 2.1 were independently changed between engine runs. A systematic approach of changing the parameters in random order was used to nullify any memory effects that could be misinterpreted in the results. 
Table 2.1: Table of Spark Parameters, symbols and physical configuration of SPDI system hardware.

\begin{tabular}{|c|c|c|c|c|}
\hline Symbol & $\begin{array}{l}\text { UI } \\
\text { Name }\end{array}$ & $\begin{array}{l}\text { Typical } \\
\text { UI } \\
\text { Value }\end{array}$ & $\begin{array}{l}\text { Physical } \\
\text { Effect }\end{array}$ & Rationale \\
\hline $\mathrm{BC}$ & $\begin{array}{l}\text { Breakdown } \\
\text { Current }\end{array}$ & 50,100 & $\begin{array}{l}\text { Determines the coil } \\
\text { charge duration be- } \\
\text { fore ignition }\end{array}$ & $\begin{array}{l}\text { The result of providing } \\
\text { the coil with excess } \\
\text { energy before ignition } \\
\text { theoretically results } \\
\text { in more energy input } \\
\text { during the arc stage of } \\
\text { the spark. }\end{array}$ \\
\hline SL & $\begin{array}{l}\text { Spark } \\
\text { Duration }\end{array}$ & $0,20,40$ & $\begin{array}{l}\text { Primary control } \\
\text { of spark duration } \\
\text { which overrides igni- } \\
\text { tion duration should } \\
\text { it be larger. }\end{array}$ & $\begin{array}{l}\text { User interface input } \\
\text { is in units of degrees, } \\
\text { which is then converted } \\
\text { by the system, using } \\
\text { the engine RPM, to } \\
\text { produce a duration. }\end{array}$ \\
\hline
\end{tabular}




\begin{tabular}{|c|c|c|c|c|}
\hline IL & $\begin{array}{l}\text { Ignition } \\
\text { Duration }\end{array}$ & $0,20,40$ & $\begin{array}{l}\text { Controls the dura- } \\
\text { tion from the igni- } \\
\text { tion signal to the } \\
\text { start of the plasma } \\
\text { portion of the spark. }\end{array}$ & $\begin{array}{l}\text { User interface input } \\
\text { is in units of degree, } \\
\text { which is then converted } \\
\text { by the system, using } \\
\text { the engine RPM, to } \\
\text { produce a duration. }\end{array}$ \\
\hline IP & $\begin{array}{l}\text { Ignition } \\
\text { Power }\end{array}$ & $\begin{array}{l}22, \quad 33 \\
44\end{array}$ & $\begin{array}{l}\text { Controls the duty cy- } \\
\text { cle of the circuit } \\
\text { transistor. For open } \\
\text { time the current is } \\
\text { allowed to flow and } \\
\text { closed time the cur- } \\
\text { rent is limited by a } \\
\text { resistor. }\end{array}$ & $\begin{array}{l}\text { Each setting is con- } \\
\text { verted into a duration } \\
\text { value in microseconds } \\
\text { however the gate times } \\
\text { have been withheld by } \\
\text { the industrial partner. } \\
\text { For now: } \\
22 \mathrm{~W}=\text { short duration } \\
33 \mathrm{~W}=\text { medium dura- } \\
\text { tion } \\
44 \mathrm{~W}=\text { long duration }\end{array}$ \\
\hline
\end{tabular}




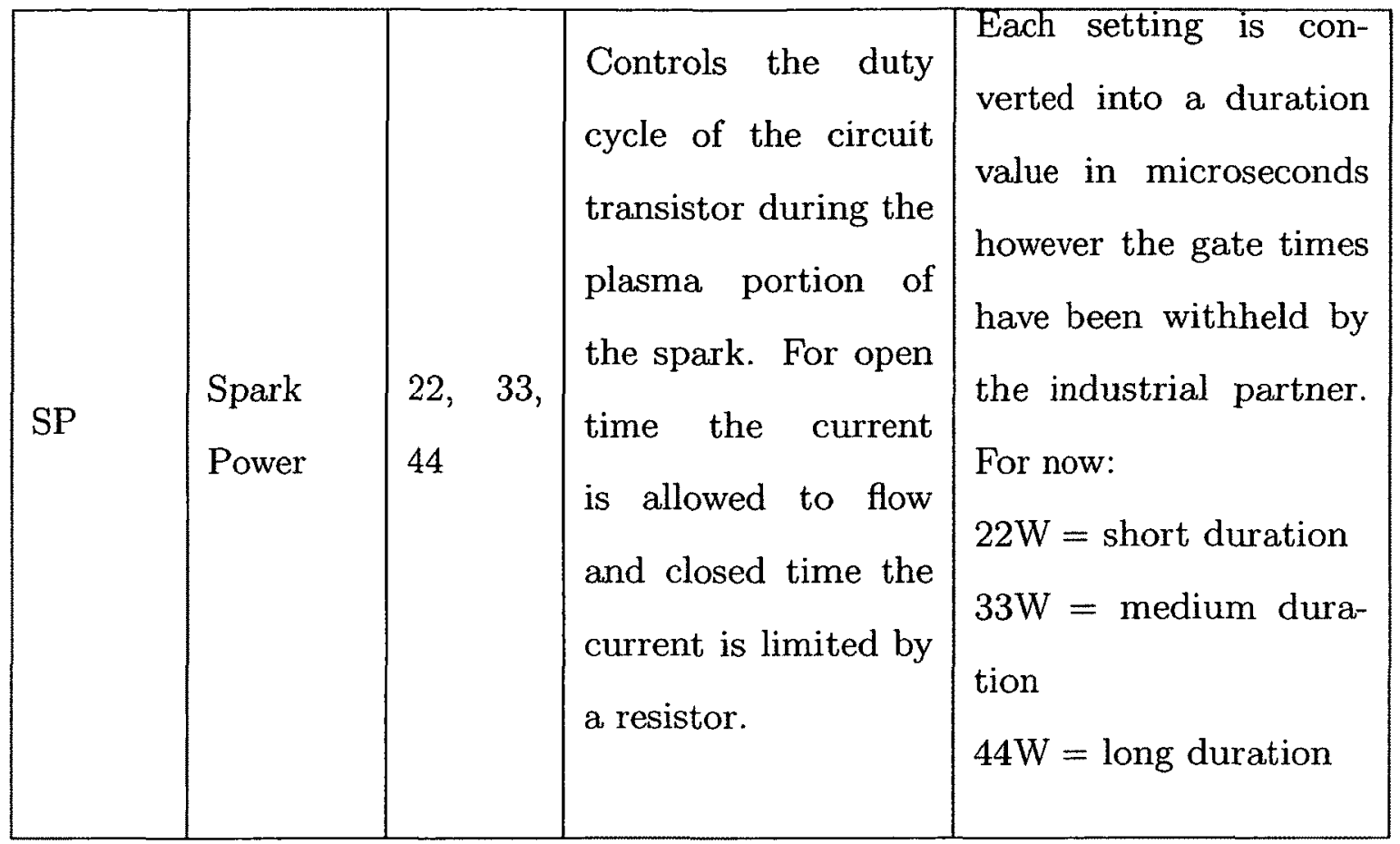

\subsubsection{Issues Resulting From Expected Curves and Actual Curves}

As described in the previous section, the parameters can be set using the ignition system interface (Figure A.2). The resulting voltage and current curves for these settings is, however, very dependent on the combustion process and therefore the resistance between the spark plug electrodes. An example of this can be seen by observing the voltage and current curves at constant pressure versus those in the running engine (Figure 2.7). The voltage inside the turbulent combustion chamber is clearly not the same as the result for constant pressure. This is attributed to the changing resistance with changing pressure and the species existing between 
the electrodes. In addition to this, the development of the plasma is also altered, and switches between the luminous and non-luminous states as will be described in Chapter 4.
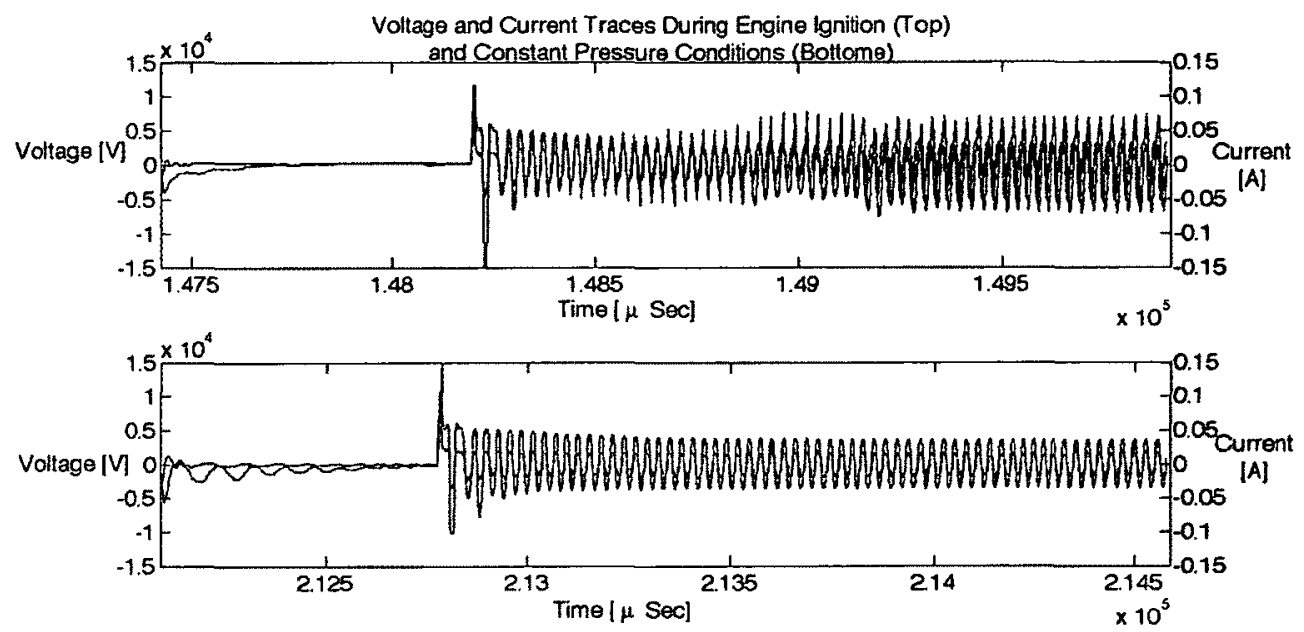

Figure 2.7: Plots of voltage and current inside a combustion chamber of a running engine (TOP) and in the constant pressure conditions (BOTTOM).

In conclusion, the SPDI unit can be set using the user interface, however the resulting voltage and current are dependent on the local conditions and were therefore monitored across all trials for as many cycles as the data acquisition system would allow. 


\section{Chapter 3}

\section{Combustion and Internal Combustion}

\section{Engines}

In addition to the background on plasma processes presented in the last chapter, an understanding of the engine geometry and terms are likewise important for this work. This section will outline the background information and geometric calculations necessary for understanding the internal combustion engine used in this study.

\subsection{Engine Geometry}

Through the use of an angular encoder affixed to the crank shaft of the engine, an approximation $^{1}$ of the piston position, velocity and acceleration can be calculated.

A list of some cylinder geometry specifications are seen in Table 3.1, as well some identified dimensions of the engine on Figure 3.1.

\footnotetext{
${ }^{1}$ This is only an approximation due to the inherent twisting of the crankshaft between the encoder wheel and the mounting point of the piston. In an ideal case, there would be a suitable mounting point for the angular encoder located on the transverse plane of the piston. However the engine used in this study did not allow for this.
} 
Table 3.1: List of engine geometry parameters and their values.

\begin{tabular}{ll}
\hline Parameter & Value \\
\hline \hline Bore $(b)$ & $68 \mathrm{~mm}$ \\
Stroke $(s)$ & $54 \mathrm{~mm}$ \\
Displacement $\left(V_{\text {Disp }}\right)$ & $196 \mathrm{~cm}^{3}$ \\
Compression Ratio $\left(C_{\mathrm{R}}\right)$ & $8.5: 1$ \\
Connecting Rod Length $(\mathrm{L})$ & $8.25 \mathrm{~cm}$ \\
\hline
\end{tabular}

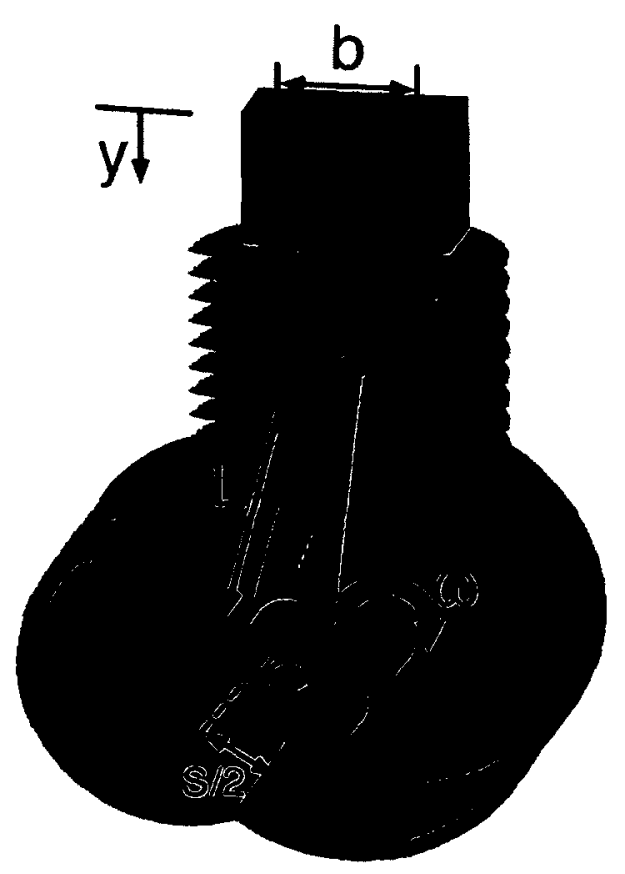

Figure 3.1: Geometric components of the cylinder; Bore $(b)$, half-stroke $(s / 2)$, crank angle $(\theta)$, piston position reference direction $(y)$. 


\subsubsection{Piston Position}

To convert the crank angle of the engine to piston position referenced from the top dead centre (TDC) position, the geometry of the connecting rod length $(L)$, stroke length $(s)$ and crank angle $(\theta)$ are used as follows;

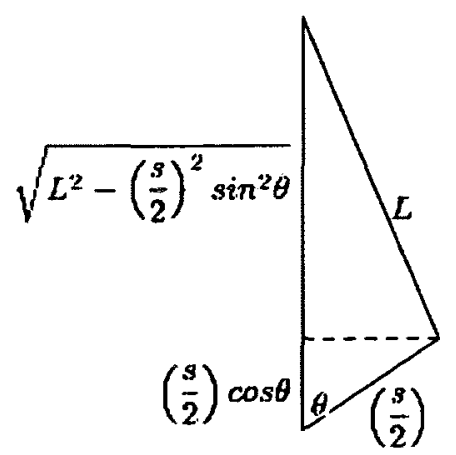

Figure 3.2: Geometry association for connecting rod, stroke length and crank angle.

Therefore the piston position can be derived as:

$$
y=\left(L+\frac{s}{2}\right)-\left(\frac{s}{2} \cos (\theta)+\sqrt{L^{2}-\left(\frac{s}{2}\right)^{2} \sin ^{2}(\theta)}\right)
$$

\subsubsection{Cylinder Volume}

Using the piston position, and the data from Table 3.1, the cylinder volume for a given piston position can be approximated as

$$
V=V_{\mathrm{CL}}+\pi\left(\frac{b}{2}\right)^{2}\left(L+\left(\frac{s}{2}\right)-y\right)
$$

where $V_{C L}$ is the volume of the chamber when the position is at TDC, derived from 
the compression ratio of the engine as follows. The displaced volume is

$$
V_{\mathrm{Disp}}=V_{\mathrm{BDC}}-V_{\mathrm{CL}}
$$

The compression ratio, $C_{\mathrm{R}}$, is

$$
C_{\mathrm{R}}=\frac{V_{\mathrm{BDC}}}{V_{\mathrm{CL}}}
$$

Rearranging Equation 17 and substituting into Equation 16 leads to:

$$
V_{\mathrm{CL}}=\frac{V_{\mathrm{Disp}}}{C_{\mathrm{R}}-1} .
$$

Using Equation 18 and Equation 15, the instantaneous cylinder volume can be calculated.

\subsubsection{Piston Speed and Acceleration}

Since the position of the piston is known, and the sampling of the data acquisition is time based, the derivatives of the position correspond to the velocity and acceleration:

$$
\begin{gathered}
v_{\mathrm{p}}=\frac{\partial y}{\partial t} \\
a_{\mathrm{p}}=\frac{\partial y^{2}}{\partial t^{2}}
\end{gathered}
$$

Therefore, both parameters can be computed for a single 4-cycle stroke. The results are seen in Figure 3.3. 


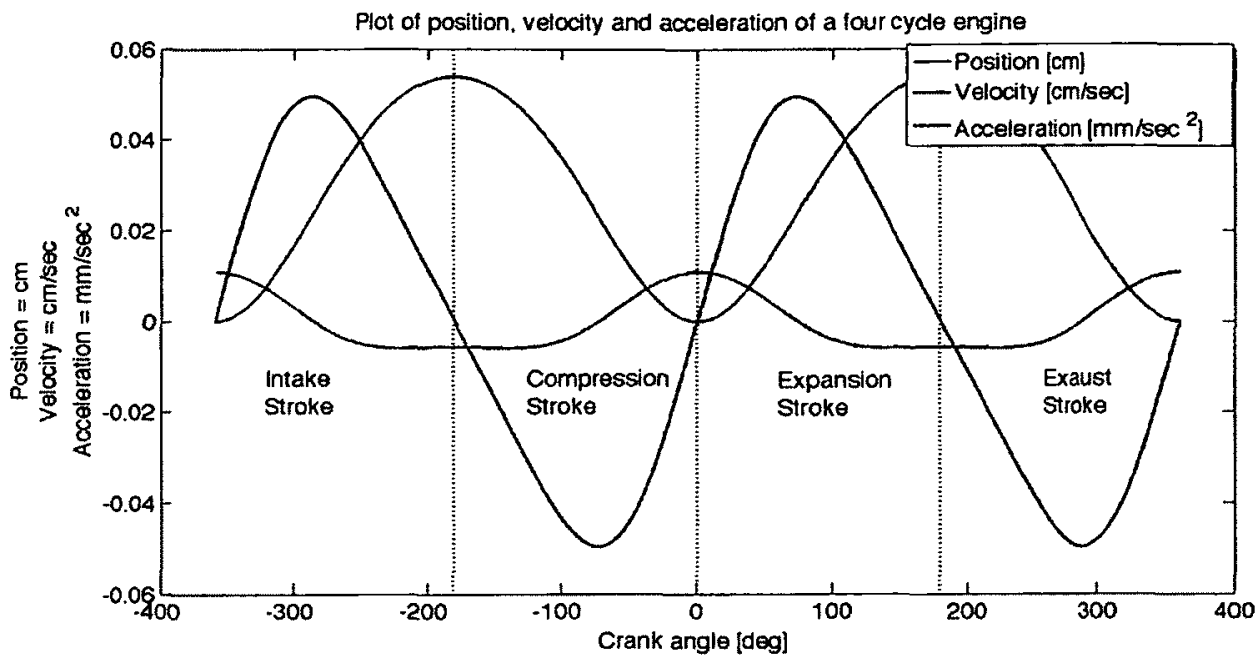

Figure 3.3: A plot of the engine resultant geometry parameters: piston position, piston speed and piston acceleration.

A transducer in the cylinder head provides a pressure curve for the engine, with the sectors identified as seen in Figure 3.4 . 


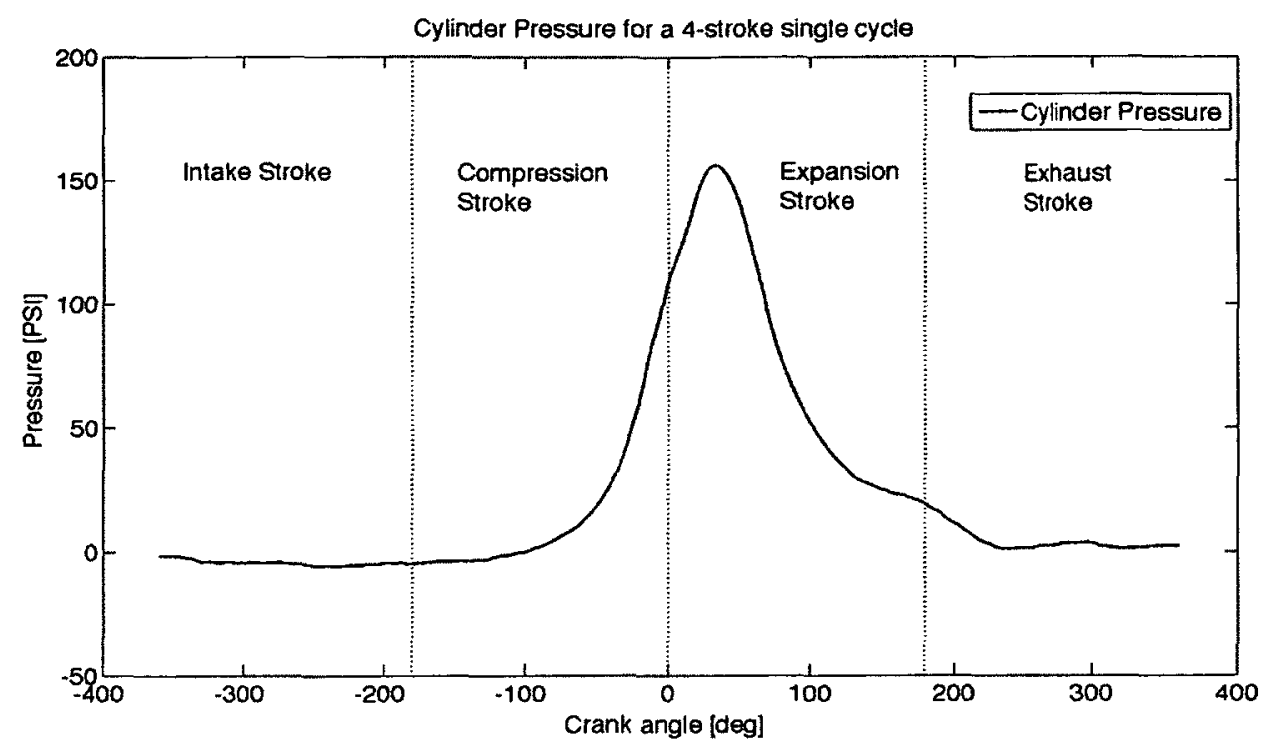

Figure 3.4: Plot of the pressure curve measured during the experiments with the appropriate strokes labelled (i.e. intake, compression, expansion, exhaust).

This type of plot (pressure vs. crank angle) serves as the primary collected data for our study.

\subsection{Engine Work}

There are three different components of the work completed by an engine cycle, positive work, negative work and net work. The general calculation for each follows as

$$
W_{1 \rightarrow 2}=\int_{V_{1}}^{V_{2}} \operatorname{Pd}(V)
$$

where $P$ is pressure and $V$ is volume. It is more useful to identify work as a function 
of crank angle in an engine, thus;

$$
W_{1 \rightarrow 2}=\int_{V\left(\theta_{1}\right)}^{V\left(\theta_{2}\right)} P d(V(\theta))
$$

These integrals are also represented in the P-V diagram (Figure 3.5). The area of the plot that is encircled by clockwise green arrows indicates the positive work of the engine and the area circled by the counter-clockwise blue arrows represents the negative work performed by the engine for pumping gases. The net work is the difference between the 2 values.

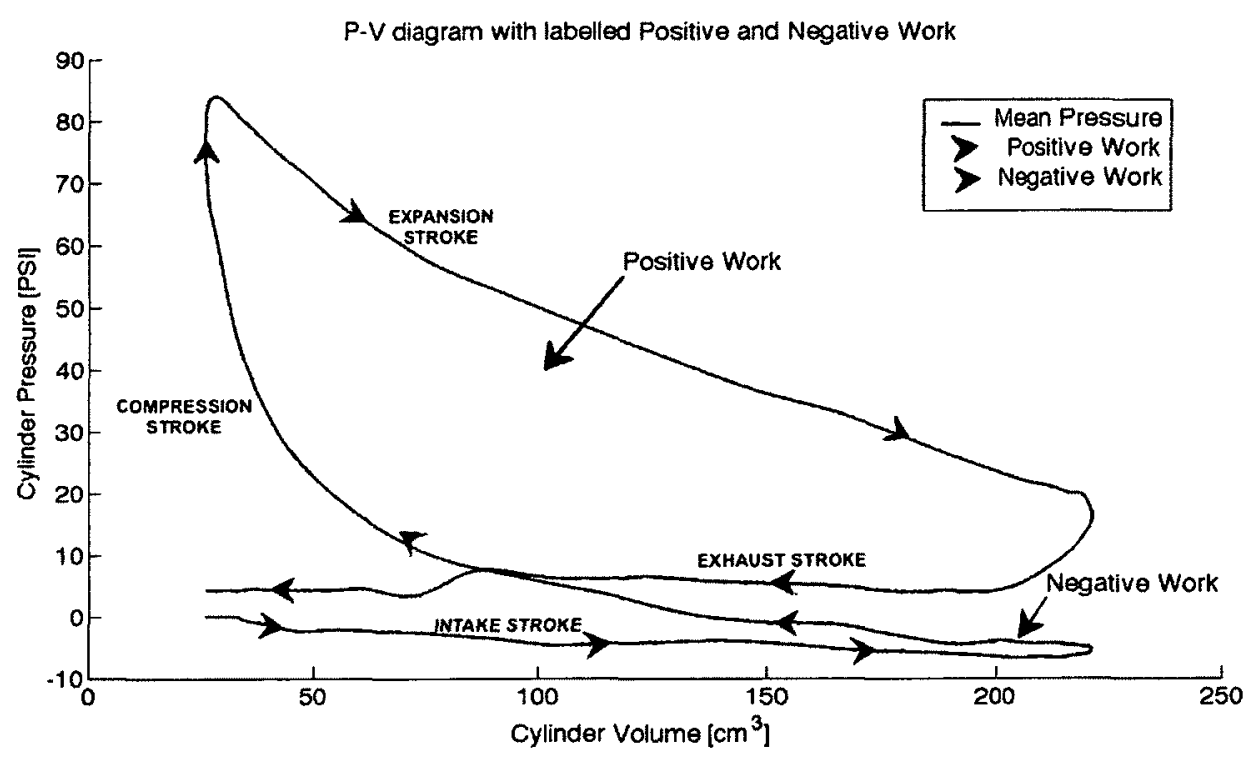

Figure 3.5: A sample P-V diagram identifying the areas for positive and negative work. 


\subsection{Mass Fraction Burn Approximation}

Three methods of calculating mass fraction burn rates were conducted in this research however for reasons outlined in this section, only one method was chosen for explaining the results in this thesis. The three mass fraction burn rate calculation use variations of the Rassweiler and Withrow [22] process. The first method assumes that a polytropic process ${ }^{2}$ is present and the first negative slope of the pressure curve represents the location corresponding to the end of combustion. The second method assumes a polytropic process is present and a combination of the first five negative slopes ${ }^{3}$ in the pressure curves corresponds to the end of combustion. The third method subtracts the motored pressure ${ }^{4}$ curve from the measured pressure curve and deduces the pressure due to combustion alone.

\subsubsection{Method 1}

The approximation of mass fraction burn inside a fixed volume combustion chamber presented by Rassweiler and Withrow [22] is used by many research groups (eg. [23], $[25],[26]$ and [27]). This method uses the relationship that the pressure rise from ignition to the end of combustion (EOC) is proportional to the fraction burnt, as

\footnotetext{
${ }^{2} \mathrm{~A}$ polytropic process is a thermodynamic process which obeys the relationship presented in Equation 24.

${ }^{3}$ Five negative slopes was chosen based on making the first method slightly more robust and resistant to signal noise. Ball et al. [23] used three negative slopes while B.Brown [24] made use of ten negative slopes for further robustness.

${ }^{4}$ The motored pressure represents the change in pressure resulting solely from the compression of the fluid in the cylinder volume during the stroke of the piston.
} 
seen in Equation 23.

$$
X_{i}=\frac{P_{i}}{P_{E O C}}
$$

where $P_{\mathrm{i}}$ is the pressure at crank angle $\theta_{i}$ and $P_{\text {EOC }}$ is the pressure at the end of combustion (EOC). Since the pressure measured at each point in the cycle is a combination of two different components, (pressure rise due to volume change and pressure rise due to combustion pressure), an approximation for the pressure rise due to volume change can be made based on the polytropic exponent before and after combustion. During these periods the pressure and volume relationship,

$$
P_{i} V_{i}^{n}=\text { Constant }
$$

where $P_{i}$ is the pressure at a given volume, $V_{i}$, and $n$ is the polytropic exponent. To calculate the pressure rise due to the change in volume only, Equation 24 is rearranged as

$$
P_{\theta, \mathrm{V}}=P_{\theta-\Delta \theta, \text { meas }}\left(\frac{V_{\theta-\Delta \theta}}{V_{\theta}}\right)^{n}
$$

If this calculated pressure is subtracted from the measured pressure, the result is the pressure rise due to combustion:

$$
P_{\theta, \mathrm{comb}}=P_{\theta, \text { meas }}-P_{\theta, \mathrm{V}}
$$

Combining Equation 25 and Equation 26, the result is the calculation of combustion pressure at the point of interest. Since the experiments by Rassweiler and 
Withrow were performed in a constant volume chamber, a normalization technique is used to reference the current combustion chamber volume to the volume at TDC:

$$
P_{\theta, \text { comb }}=\left(P_{\theta, \text { meas }}-P_{\theta, \mathrm{V}}\right)\left(\frac{V_{\theta}}{V_{\mathrm{TDC}}}\right)^{n} .
$$

Finally combining Equation 23 and Equation 27, a calculation for the mass fraction burned can be conducted as,

$$
X_{\theta}=\frac{\sum_{i=\theta_{\text {Spark }}}^{i=\dot{\theta}} P_{i, \mathrm{comb}}}{\sum_{j=\theta_{\text {Spark }}}^{j=\mathrm{EOC}} P_{j, \mathrm{comb}}} .
$$

Therefore, the mass fraction burned at each angular location, representing each equally spaced value of $i$, in the cycle between the ignition and the estimated end of combustion is an element of $X_{\theta_{\mathrm{Spark}}}, X_{\theta_{2}}, X_{\theta_{3}}, \ldots, X_{\theta_{\mathrm{EOC}}}$. To make use of this procedure, a reasonable approximation of the polytropic exponent is necessary, as well as an accurate estimate for the end of combustion location.

\section{Estimation of the Polytropic Exponent}

The derivation of the polytropic exponent as performed by Rassweiler and Withrow is rendered from the portion of the pressure rise prior to ignition for the compression stroke and from the portion of the pressure curve after the end of combustion, for the expansion stroke. Linear interpolation is then used for the period during which combustion has occurred. Other researchers have proposed methods to make this approximation more accurate (Shayler et al. [28], Cheung and Heywood [29]), as 
well as determined procedures and weighted effects that cause deviations in the MFB analyses solely due to the choice in polytropic indices Brunt and Pond [30]. However, Ball et al. [23] have outlined most of the MFB processes that are used in research today and therefore a basic model of constant polytropic index is chosen for this research, for both the compression and expansion portions of the pressure data, $(n=1.4)$. As further advancement, and such will be included in any further release of the software developed here, the basic model will have $n=1.4$ for $\theta \leq T D C$ and $n=1.0$ for $\theta>T D C$. These approximations presented by Shayler et al. [28] represent the isentropic compression process before TDC $(n=1.4)$ and an isothermal expansion process after TDC until EOC $(n=1.0)$. Future work for this project would include using the test apparatus to generate more pressure data that can in turn be used to compute a more accurate polytropic index for this engine taking into account piston blow-by and thermal properties of the cylinder.

\section{End of Combustion Location}

In Method 1 the derivatives between each point of the pressure curve are computed. Then the first location of a negative slope is considered the end of combustion. However, this method is very susceptible to signal noise, and thus the employment of Method 2, as will be discussed, is chosen for this analysis.

\subsubsection{Method 2}

Method 2, differs from Method 1 by changing the estimation of EOC location. The methods and equations prescribed in Section 3.3.1 are identical to those used in Method 2, except that when estimating the EOC location a sequence of five negative 
slopes (instead of one) are used to discern the end of combustion location. This method, while computationally more demanding, is a more robust version of Method 1 against signal noise.

\subsubsection{Method 3}

Method 3, differently from that of Method 1 and 2, uses the motored pressure traces measured using the experimental apparatus discussed in Section 5.2. Instead of approximating the polytropic indices of the gases inside the combustion chamber, the compression and expansion pressures resulting from the moving piston are measured. The measurements are then subtracted from the ignited pressure trace and the result is the pressure rise due to combustion only. The mass fraction burn analysis is conducted using Equation 27 and Equation 28, where the $P_{\theta, v o l}$ is measured as opposed to calculated.

\subsubsection{Conclusions}

While the three methods presented here are all used by researchers today, when conducting the analyses it was determined that Method 2 was the most suitable for our research team's application. The choice to use Method 2 was due to difficulties in using Method 3 on a single cylinder engine, and due to the greater robustness of Method 2 against signal noise as compared to Method 1.

In Method 1, the signal noise was determined to be the largest contributor to false identification of EOC locations. This was however easily rectified by using Method 2's process of choosing EOC, and therefore making Method 1 of little to no use. 
The cost benefit of extra computational power versus errors in EOC location was determined by the research group to be worthwhile and thus Method 2 was chosen over Method 1.

In Method 3 there exists perturbations in the resulting pressure rise due to combustion. This can be seen in Figure 3.6, where the cylinder pressure begins to rise away from its motored pressure curve prior to ignition. If it were only a single event, this could be a result of engine ping ${ }^{5}$, although its reoccurrence has brought the conclusion that our measurements of motored pressure curves simply do not have adequately refined TPS and RPM increments. When the motored pressure curves were collected, it was performed in a step-wise manner where the RPM was varied at multiple throttle positions. The result of this however was that not all TPS positions were collected, due to the increments being too large. This can be rectified for future projects by using the test apparatus developed here and decreasing the TPS increments.

\footnotetext{
${ }^{5}$ Engine ping is a term describing the event where the air fuel mixture self-ignites prior to the spark ignition event.
} 


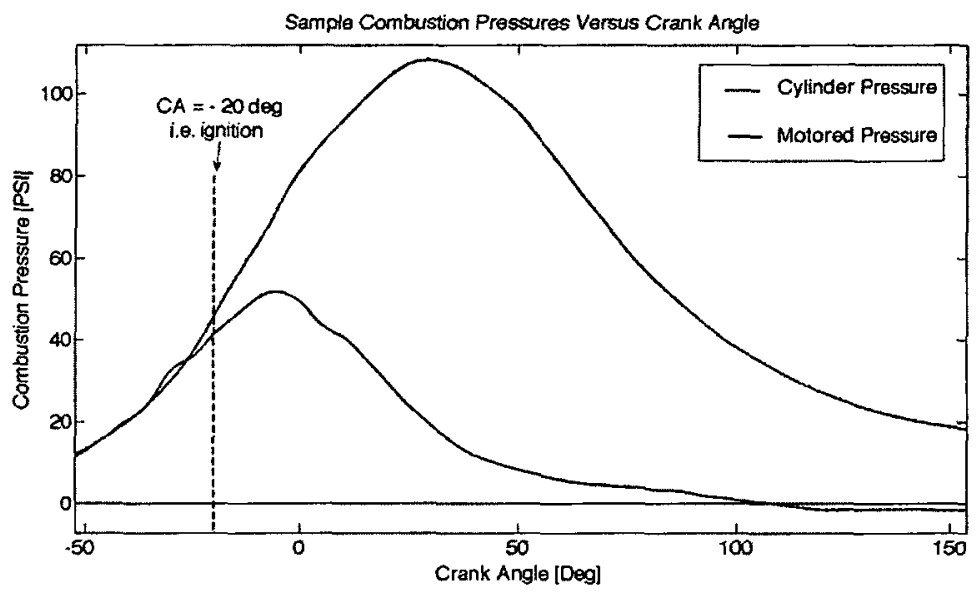

Figure 3.6: An example cylinder pressure versus its appropriate motored pressure. The deviation of the cylinder pressure from the motored pressure exists prior to ignition of the mixture. 


\section{Chapter 4}

\section{Experimental Methods and Results for Imaging the Ignition Systems}

\subsection{Purpose of Experiment}

Understanding the characteristics of the plasma which is created by the ignition device in this investigation is important for understanding whether it carries the potential to affect the combustion process. For this reason, an optical investigation is used at ambient pressure and at pressurized conditions. The purpose of these experiments was to gather images and voltage-current traces of the SPDI system at multiple settings, as well as images of the inductive discharge, and make conclusions based on their comparison. 


\subsection{Apparatuses of Experiments}

\subsubsection{Ambient Pressure Experiment}

The first experiment was conducted at ambient conditions. The apparatus included the following equipment;

- NanoSense MkII CCD High Speed camera from Dantec Dynamics

- Nikkon AF Micro-Nikkor 60mm (f/2.8 D) Lens

- MSO-9201 Oscilloscope with a logic analyzer from Link Instruments

- National Instruments NI-9178cDAQ board equipped with a NI-9401 DAQ card for triggering

- Spatial plasma discharge ignition system

- Two copper electrodes with beveled corners and a flat tip, with tip diameter of $2 \mathrm{~mm}$

The two electrodes were mounted horizontally on the work bench and the CCD camera was oriented perpendicular to the discharge gap. The ignition system, CCD camera and oscilloscope were all triggered using the National Instruments DAQ system. Due to the highly manipulable gating times and frame rate of the CCD camera, all three triggered components used the same trigger pulse. The spark gap of $10 \mathrm{~mm}$ was arbitrarily chosen for image quality and kept constant for all trials. 


\subsubsection{Pressurized Experiment}

The second apparatus require the manufacturing of a pressure vessel, as shown schematically in Figure 4.1 with the important items labelled.

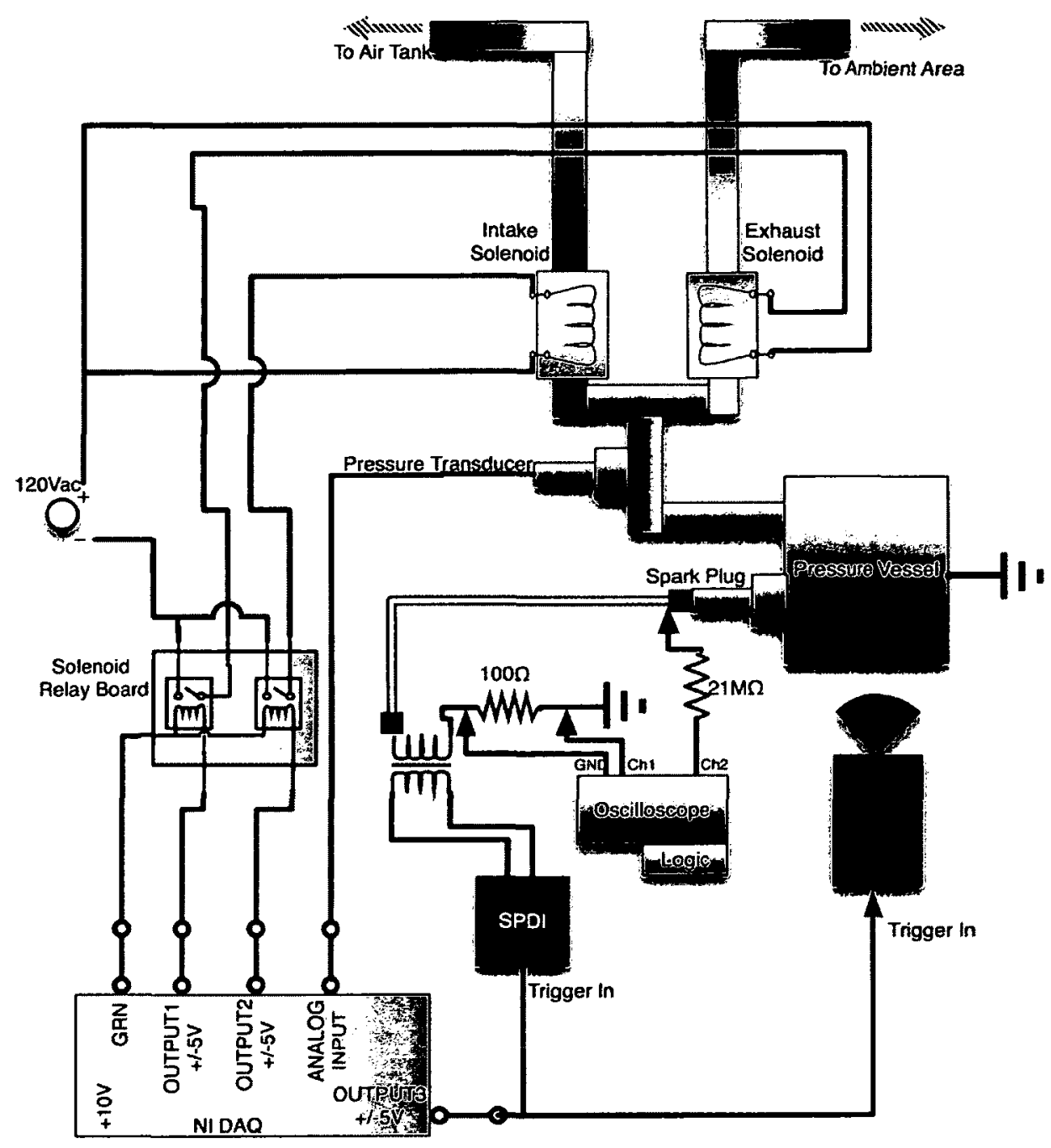

Figure 4.1: The imaging setup for pressurized conditions.

The CCD camera was oriented perpendicular to the discharge gap that is located 
inside the pressure vessel. The electrodes used in the experiment are those of a NGK BCPR6EGP spark plug, which is also used in the combustion experiments. The discharge gap remained constant throughout all trials and was thus $0.889 \mathrm{~mm}$ or $0.035 "$, the diameter of the top electrode is $0.89 \mathrm{~mm}$ and the bottom electrode is $1 \mathrm{~mm}$ thick rectangular.

\subsection{Ambient Pressure}

Through this experiment it was deduced that the plasma assisted ignition device did provide a plasma conduit where ionized particles travelled through an arc discharge as well as a cloud. This is more easily described by Figure 4.3, where there exists no arc conduit compared to that of Figure 4.2. This is because in Figure 4.2, the frame exposure began before the breakdown current and extended over the length of the entire spark, while in Figure 4.3 the exposure started after the breakdown current and extended to the end of the spark event.

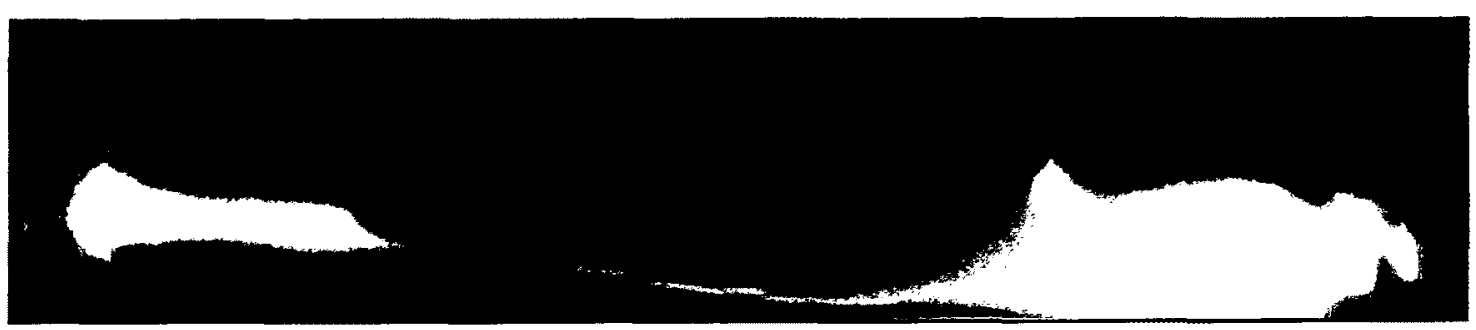

Figure 4.2: This image corresponds to a single frame of the acquisition where breakdown is included showing the conduit of ionization as the magnetic field collapses the ionized particles into a single arc.

When using one exposure to image the inductive discharge unit, the result is seen in Figure 4.4, comparing this image to those of the SPDI unit, the difference is 


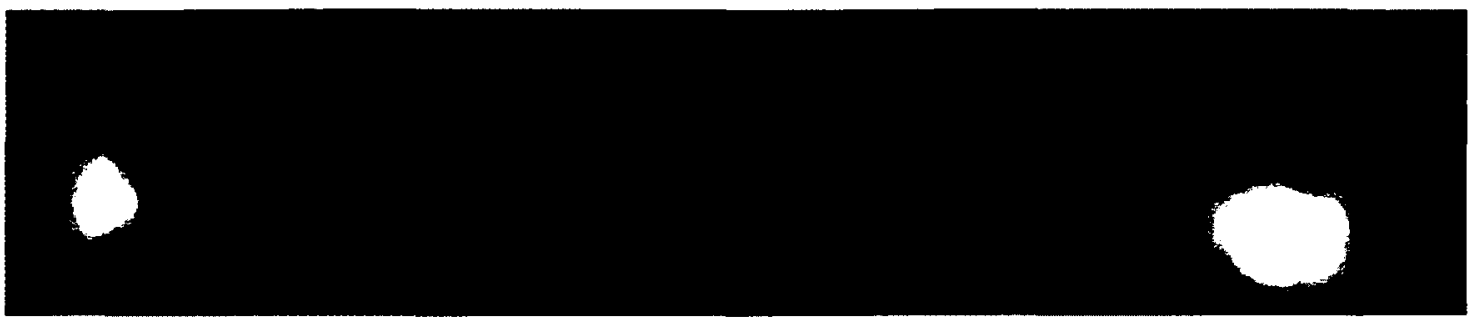

Figure 4.3: This image corresponds to a single frame of the acquisition where the breakdown current was not included, this represents the oscillating voltage of the ignition device creating the plasma.

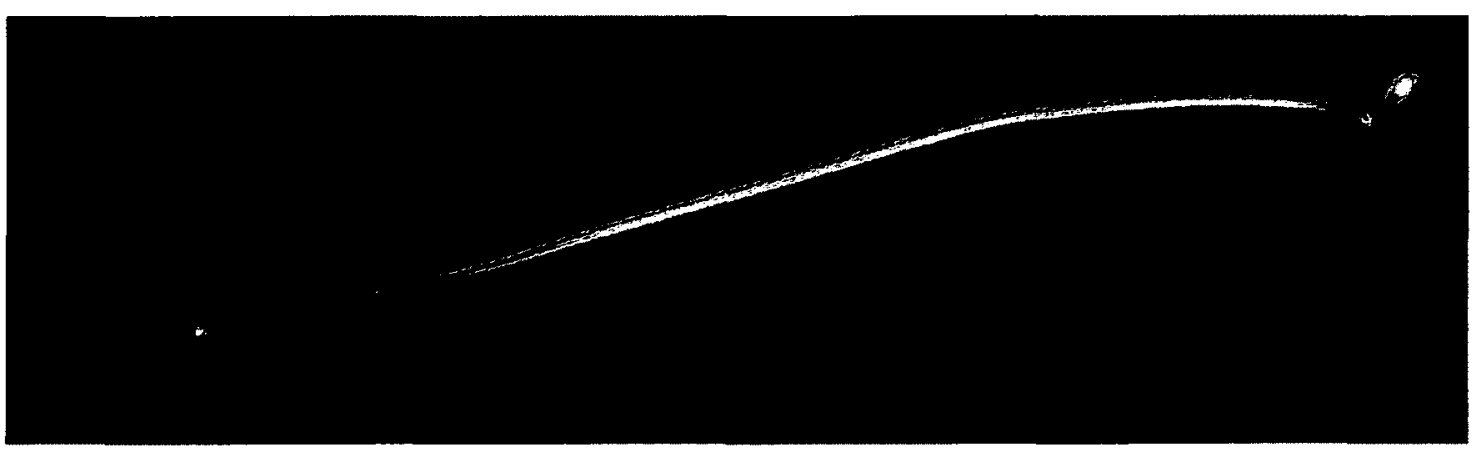

Figure 4.4: This image corresponds to a single frame of the acquisition for the Inductive Discharge, it is apparent that the arc has been formed and the magnetic field has collapsed the ionized particles into the concentrated conduit.

obvious.

In conclusion, the SPDI spark has been shown to create the postulated plasma state after the formation of the arc discharge. This plasma state was then sustained for the duration of the spark event. The inductive discharge accomplished the arc formation, which collapsed upon removal of the voltage source, as expected. 


\subsection{Discharge at Pressures Greater Than Atmo- spheric}

The experiment using the pressurized vessel highlighted a few interesting points regarding the plasma discharge. Firstly, depending on the breakdown current the luminosity of the arc conduit between the electrodes varied, and secondly, it was discovered that there are two states for the plasma discharge, one luminous and one non-luminous. These will be discussed further in the following sections.

\subsubsection{Breakdown Current Versus Pressure}

When experimenting with the breakdown current setting, it was seen that a difference in the region of luminosity, or spectral intensity, occurs as a function of breakdown current and pressure. This can be more clearly seen in Figure 4.5 where the intensity decreases with breakdown current and increases with pressure. 

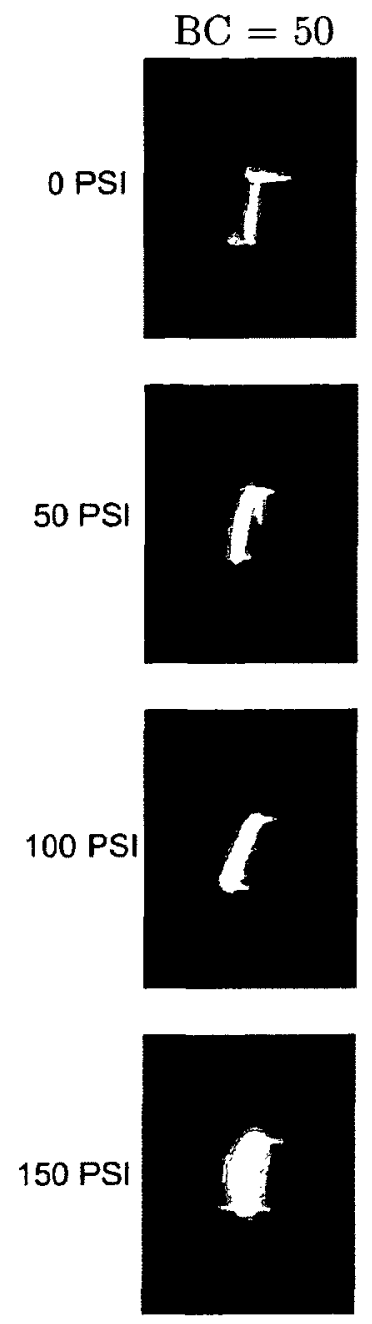
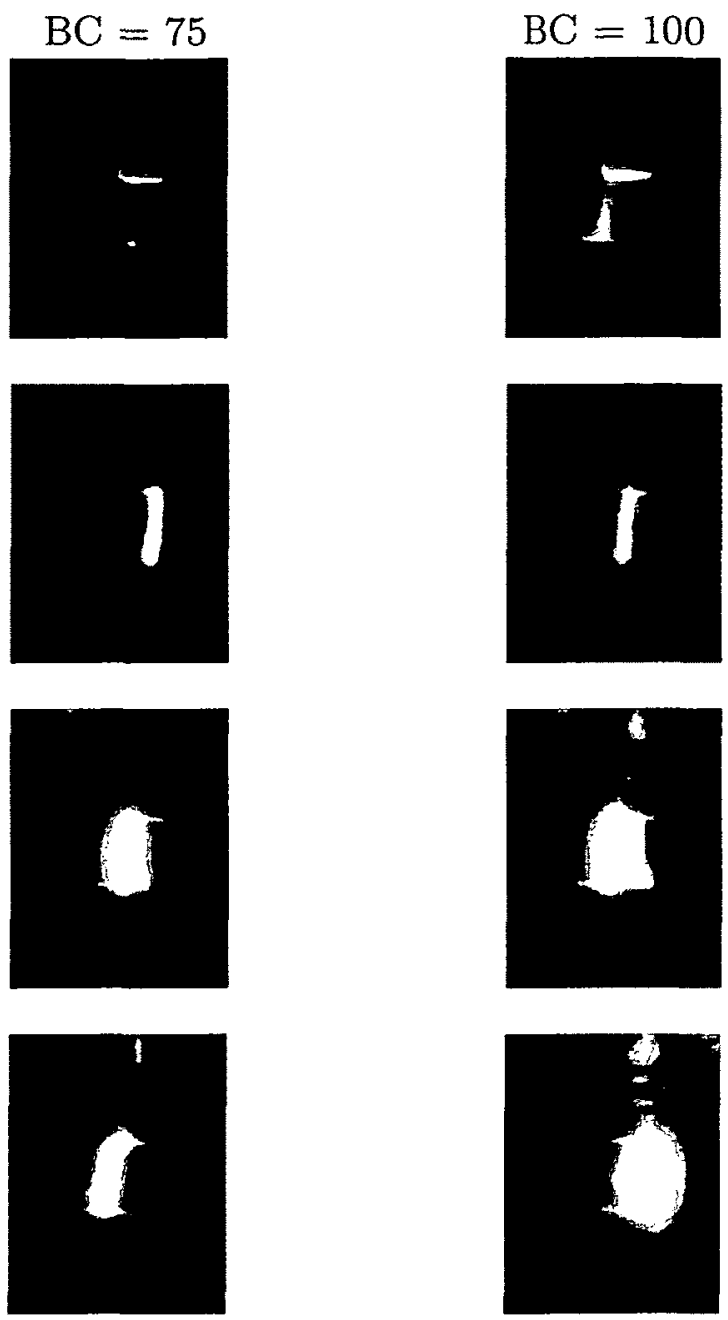
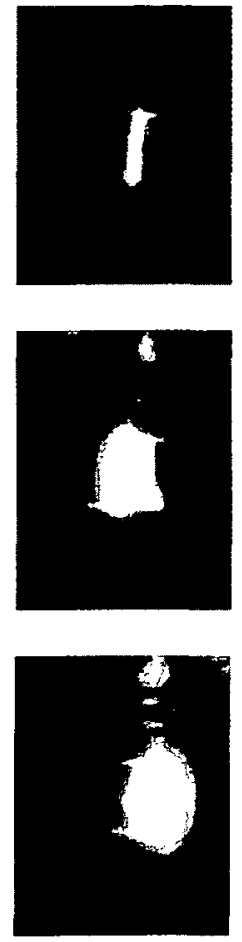

Figure 4.5: From left to right, $\mathrm{BC}=50,75$ and 100. The rows (top to bottom) correspond to variable pressure (0 PSI, 50 PSI, 100 PSI and 150 PSI).

The results found here were further investigated when $\mathrm{BR}^{1}$ was held constant at 100 and the pressure was increased from 0 PSI to 150 PSI in 10 PSI increments. The results are shown in Figure 4.6, where the luminosity increases with pressure.

\footnotetext{
${ }^{1}$ Please recall Table 3.1 , where the notation of $\mathrm{BC}$, which is the "breakdown current" setting on the SPDI device, is defined.
} 


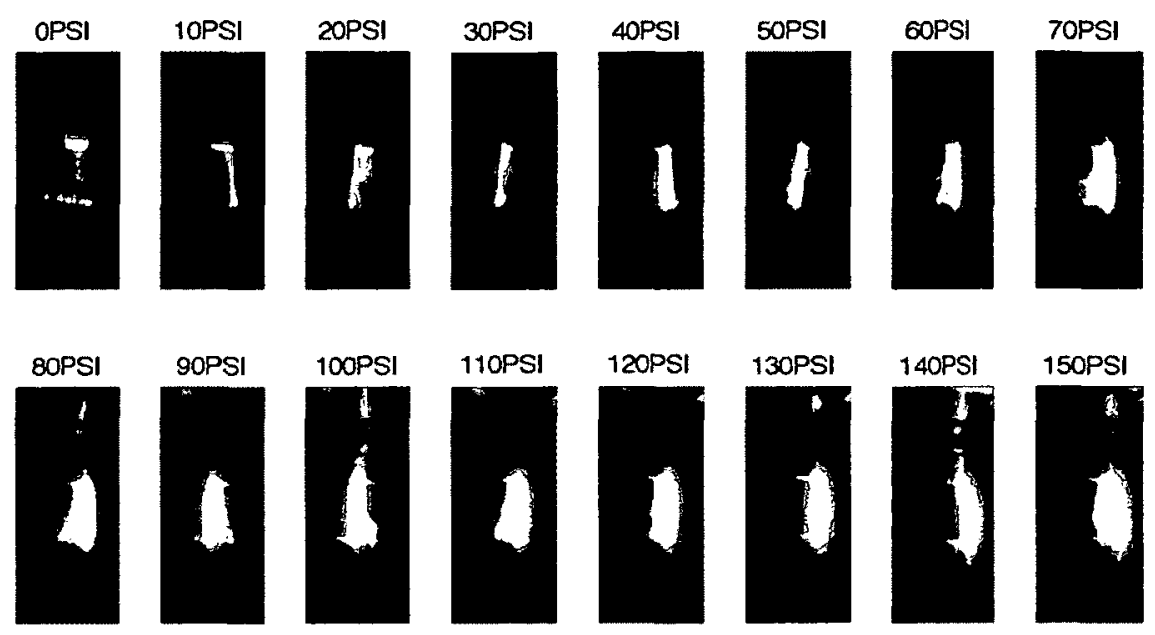

Figure 4.6: Images of the first $260 \mu \mathrm{Sec}$ of discharge, the frames represent increasing pressure with constant SPDI spark breakdown current settings. The spark profile has the following settings: $\mathrm{BC}=100, \mathrm{SL}=40, \mathrm{IL}=40, \mathrm{SP}=44, \mathrm{IP}=$ 44.

\subsubsection{Constant Plasma Settings With Incremental increases in Pressure}

Figure 4.7 and Figure 4.8 show that when increasing the pressure inside the vessel at 10 PSI increments, it can be clearly seen that the images follow the trends previously discussed in Section 4.4.1, where the luminosity increases with pressure for the first frame of each discharge. However, another noticeable change in the plasma discharge is the lack of luminosity for pressures exceeding 100PSI. 


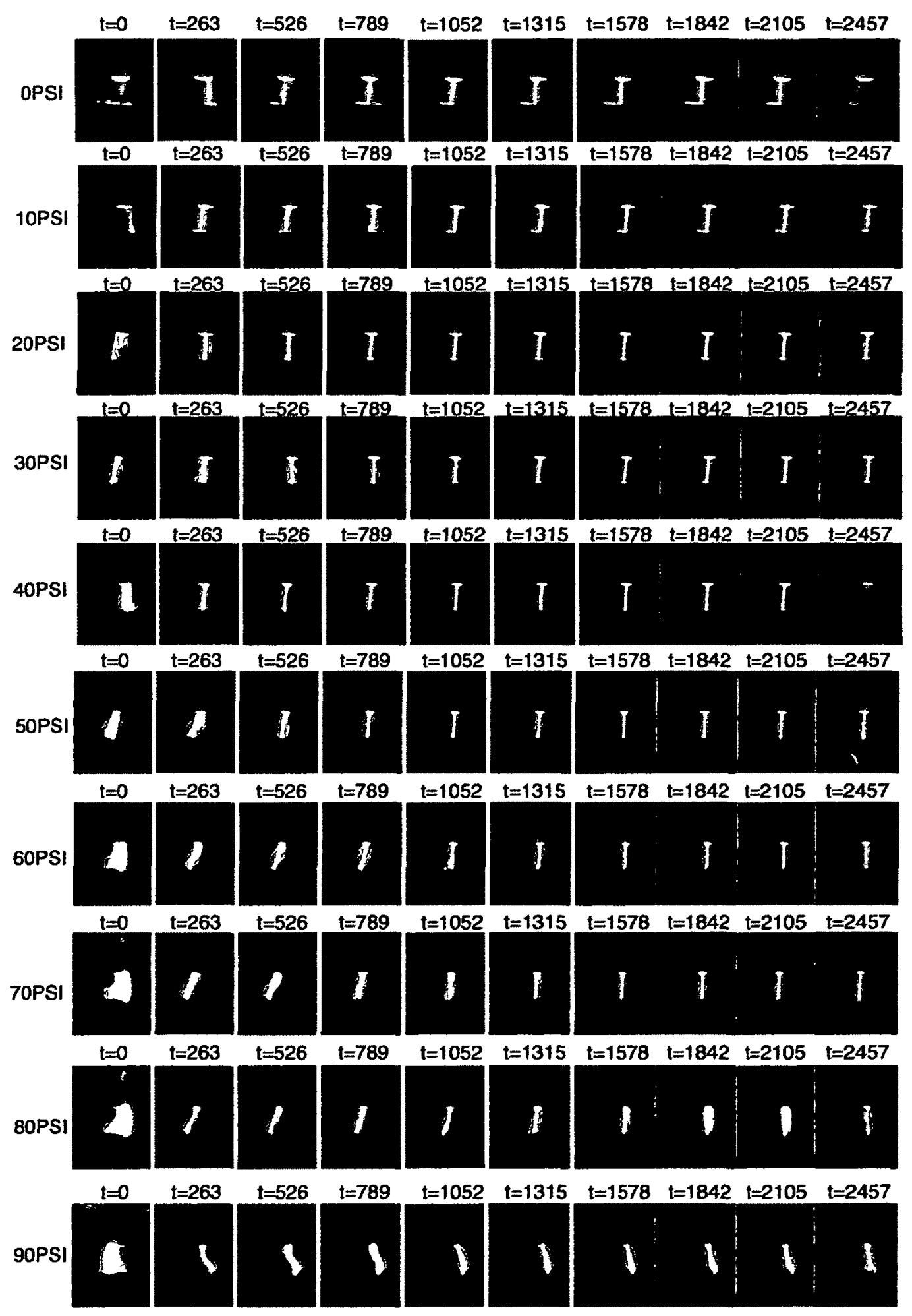

Figure 4.7: Photographs taken from 0 PSI to 90 PSI at 10 PSI increments, camera gate time of $260 \mu \mathrm{Sec}$ at a frame rate of $3800 \mathrm{~Hz}$, spark profile is; $\mathrm{BC}=100$, $\mathrm{SL}=40, \mathrm{IL}=40, \mathrm{SP}=44, \mathrm{IP}=44$. All time labels are in microseconds. 


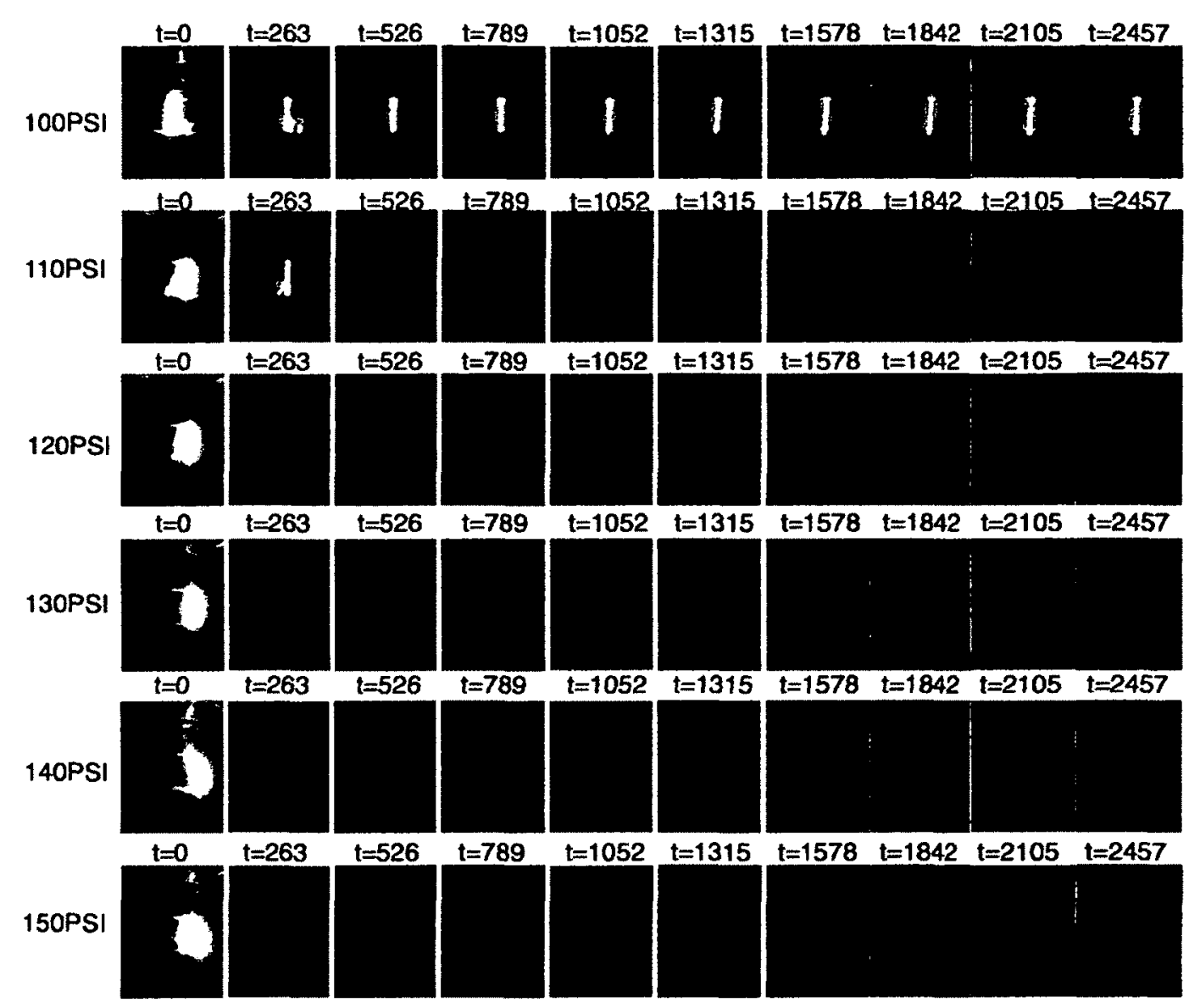

Figure 4.8: Photographs taken from 100 PSI to 150 PSI at 10 PSI increments, camera gate time of $260 \mu \mathrm{Sec}$ at a frame rate of $3800 \mathrm{~Hz}$, spark profile is; $\mathrm{BC}=$ $100, \mathrm{SL}=40, \mathrm{IL}=40, \mathrm{SP}=44, \mathrm{IP}=44$. All time labels are in microseconds.

Figure 4.9 and 4.10 correspond to the voltage and current traces for each collection of images in Figures 4.7 and 4.8. It can be seen that the voltage and current traces show little response to the changes in pressure from 0 to 90 PSI. However this changes above 90 PSI, the first few pulses at 100PSI show small peaks in current. This is true for 110PSI as well, however there is a substantial increase in the amount of current flowing through the discharge later into the spark event. This is again 
seen in all cases above 110PSI (Figure 4.11) where the current peaks are much larger compared to the current peaks at pressures below 100PSI. Comparing this to their corresponding images in Figures 4.7 and 4.8, the discharges appear as being nonluminous at pressures above 110PSI. From these observations, it can be concluded that the plasma exhibits two different discharge types dependent upon pressure. 
OPSI

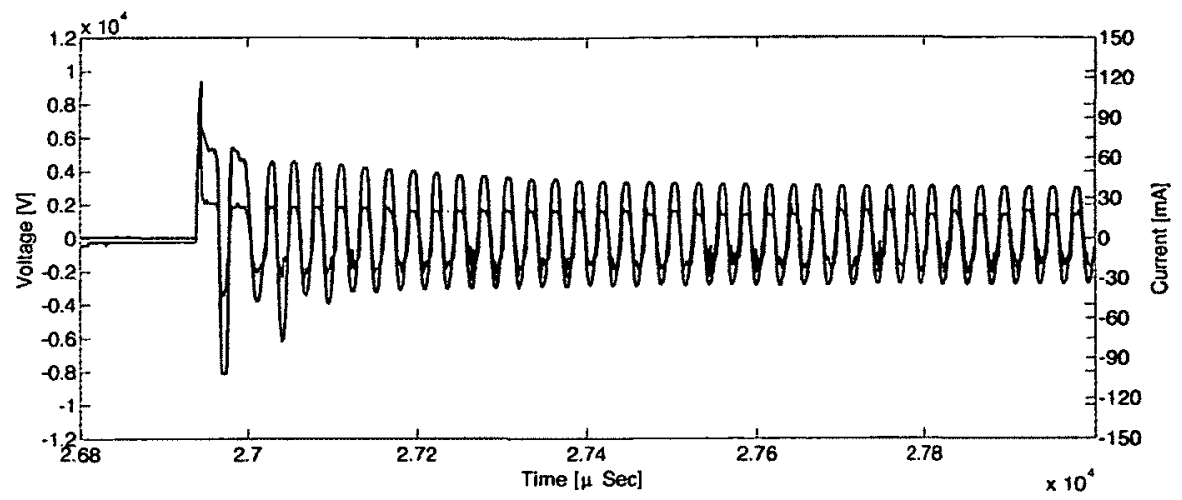

10PSI
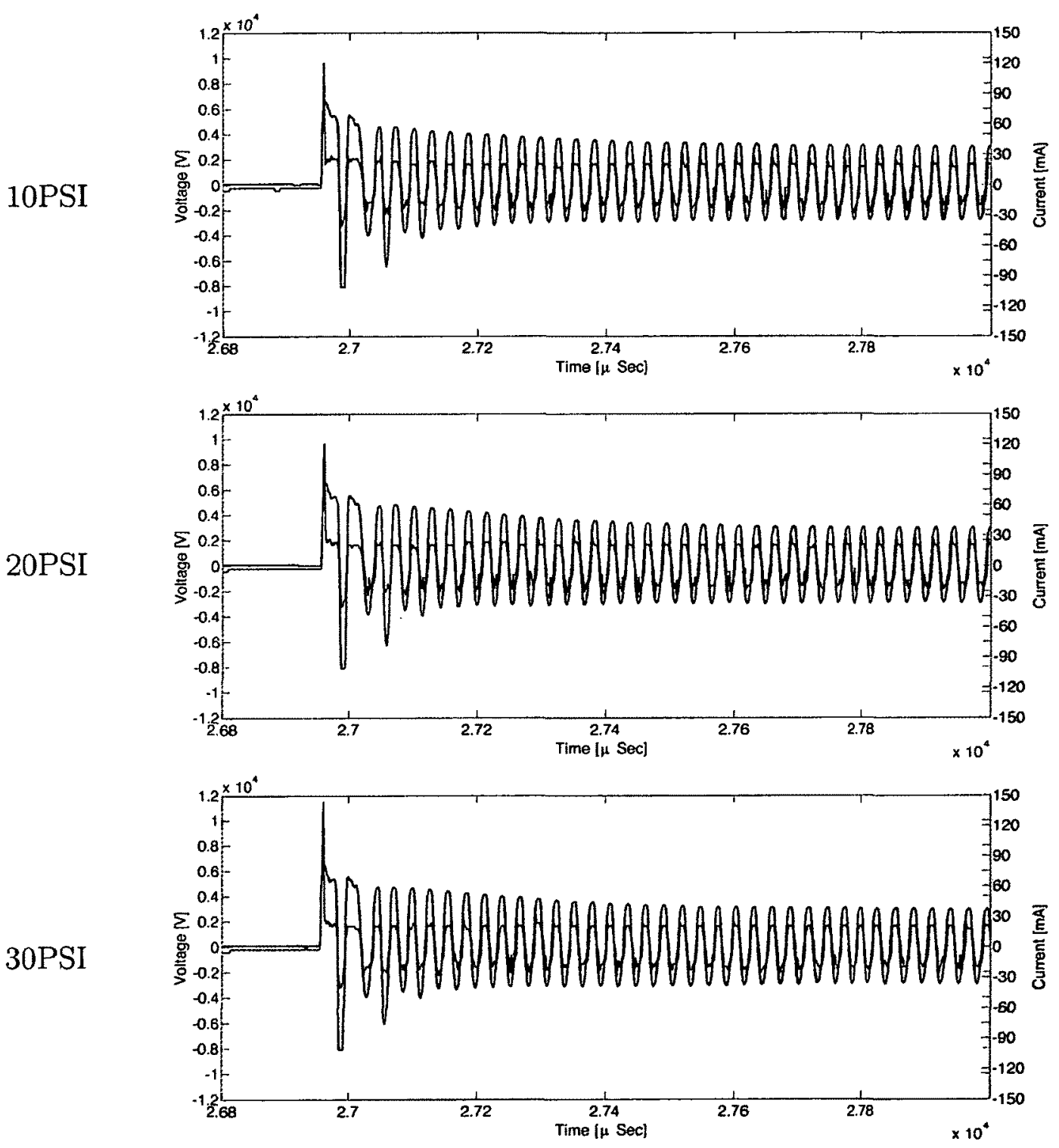

Figure 4.9: Discharges measured from 0 PSI to 50 PSI at 10 PSI increments, spark profile is; $\mathrm{BC}=100, \mathrm{SL}=40, \mathrm{IL}=40, \mathrm{SP}=44, \mathrm{IP}=44$. (See the top image in Figure 4.13 for an enhanced view of the red dashed section outlined in the 40PSI Figure here.) 

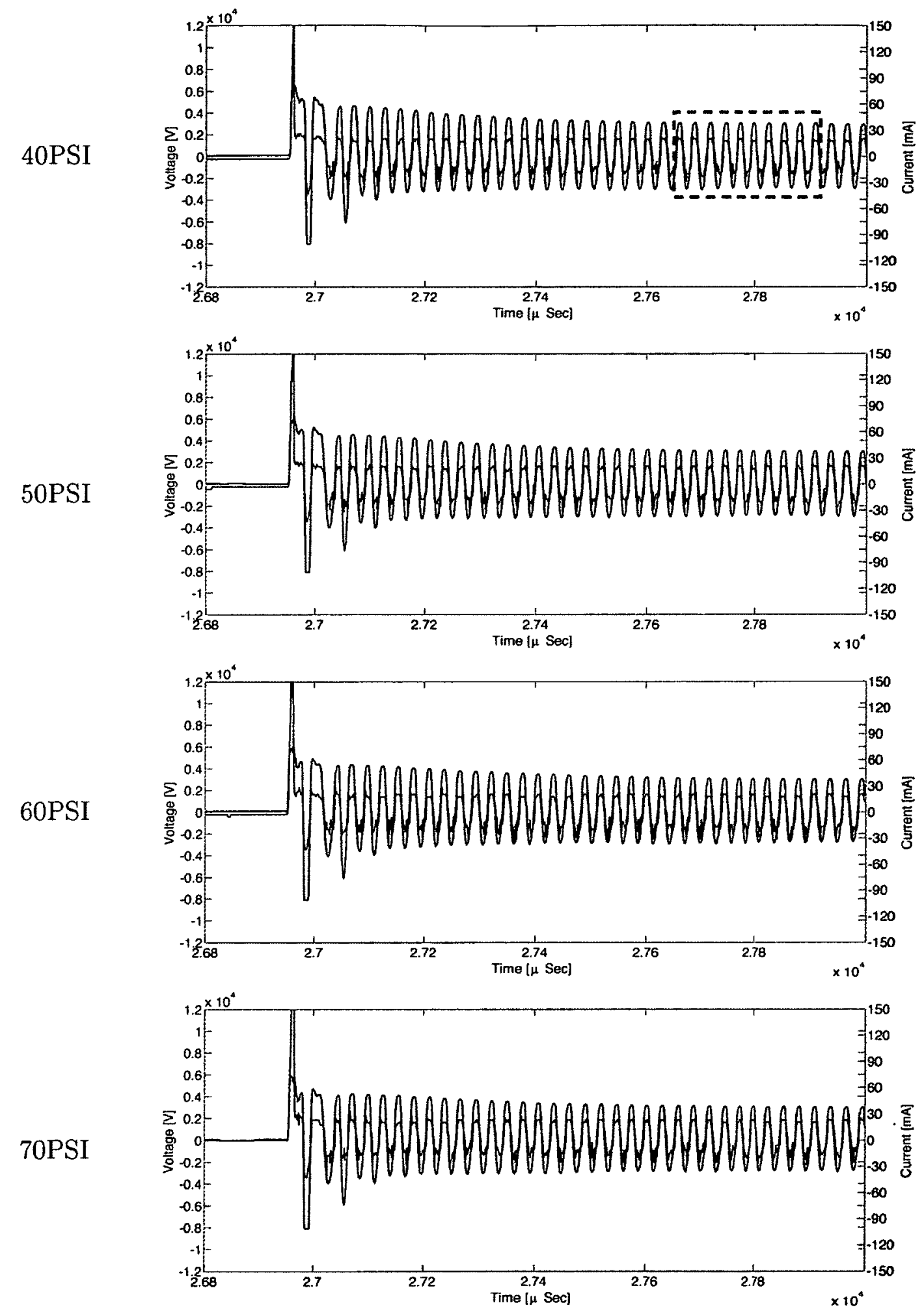

Figure 4.10: Discharges measured from 40 PSI to 70 PSI at 10 PSI increments, spark profile is; $\mathrm{BC}=100, \mathrm{SL}=40, \mathrm{IL}=40, \mathrm{SP}=44, \mathrm{IP}=44$. 

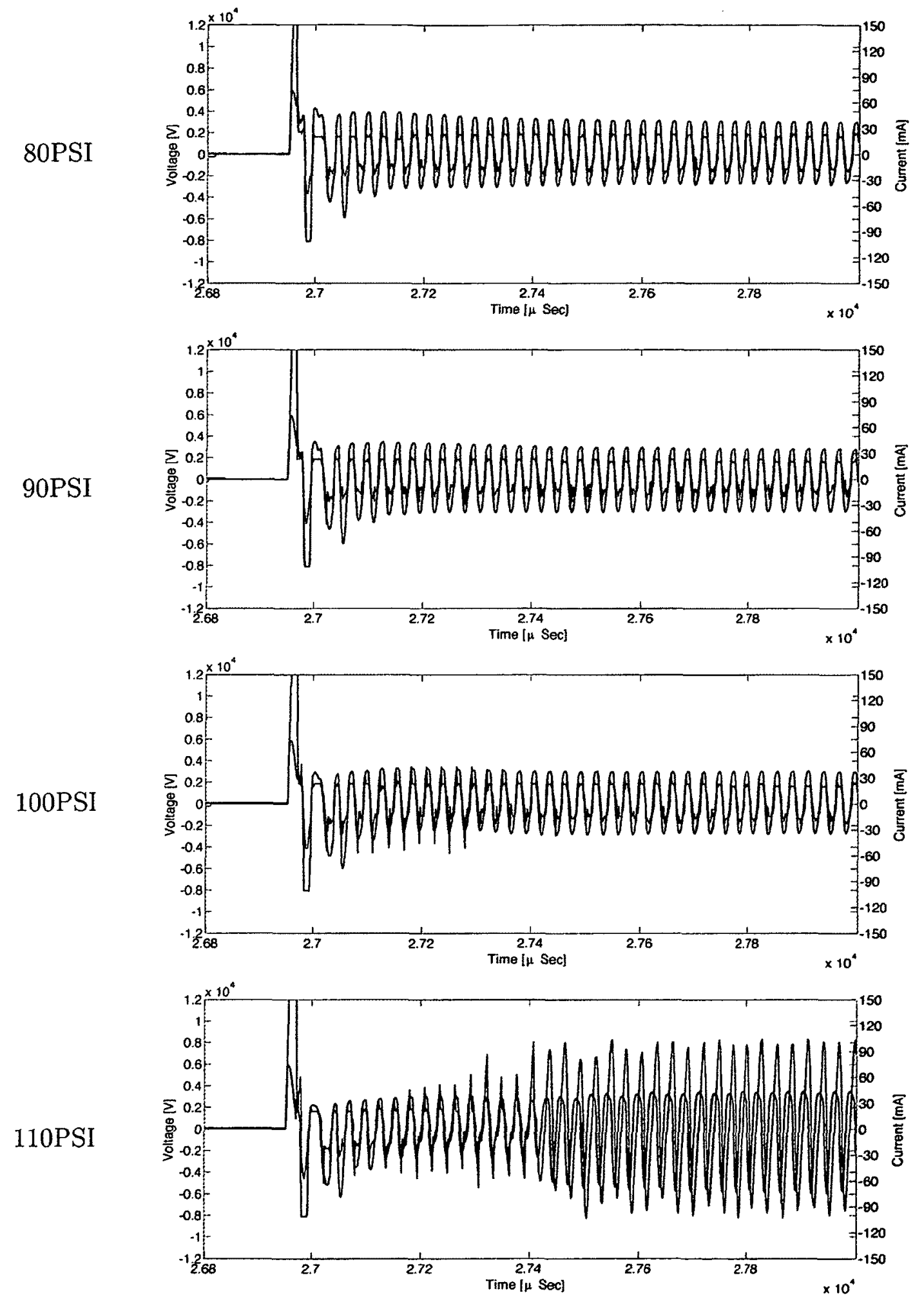

Figure 4.11: Discharges measured from 80 PSI to 110 PSI at 10 PSI increments, spark profile is; $\mathrm{BC}=100, \mathrm{SL}=40, \mathrm{IL}=40, \mathrm{SP}=44, \mathrm{IP}=44$. 

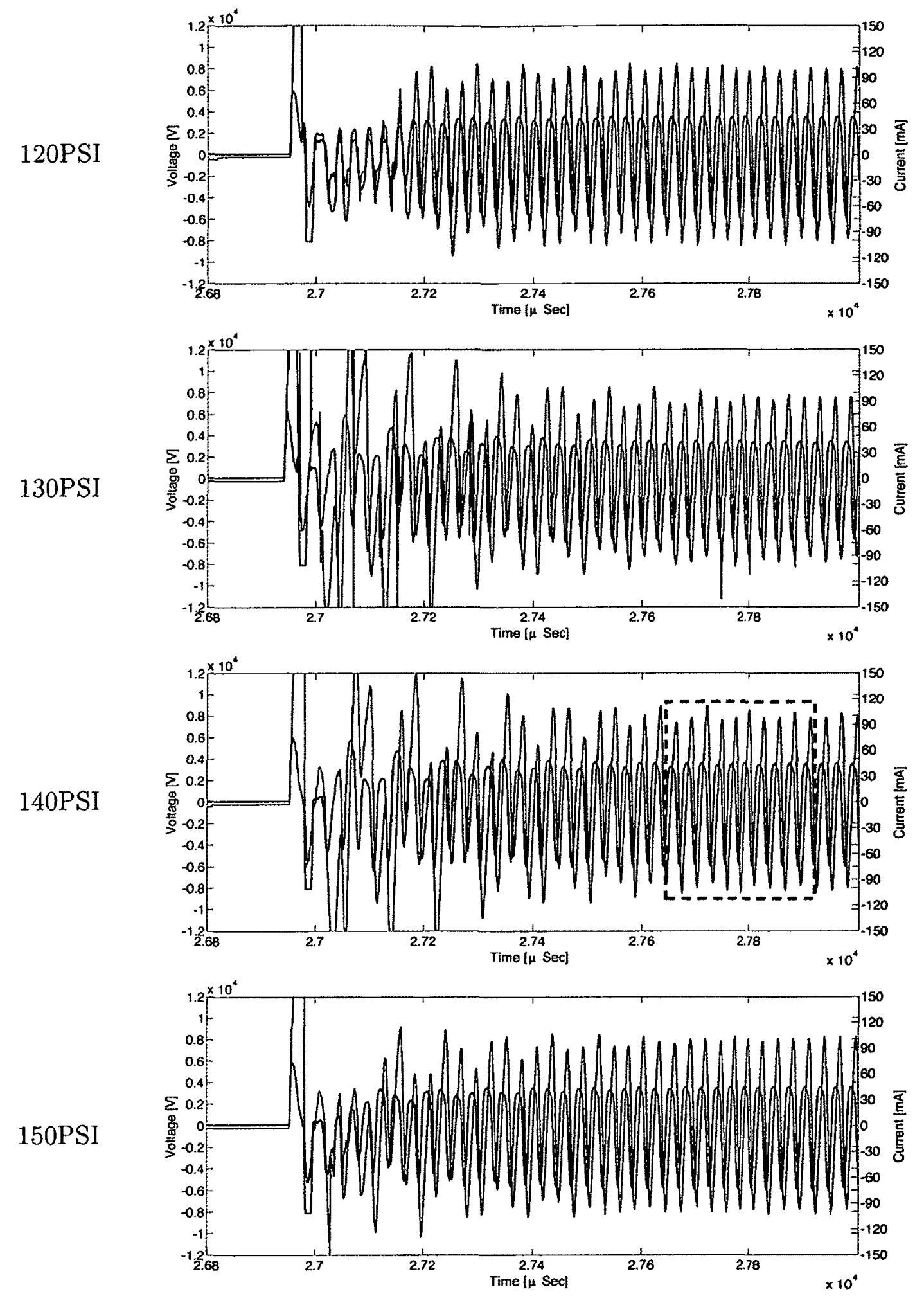

Figure 4.12: Discharges measured from 120 PSI to 150 PSI at 10 PSI increments, spark profile is; $\mathrm{BC}=100, \mathrm{SL}=40, \mathrm{IL}=40, \mathrm{SP}=44, \mathrm{IP}=44$. (See the bottom image in Figure 4.13 for an enhanced view of the red dashed section outlined in the 140PSI Figure here.) 
Taking a closer look at a few oscillations (Figure 4.13) for both types of discharge, the luminous (top) and non-luminous (bottom), we can see that the voltage and current frequency is phase shifted by 90 degrees. The non-luminous nature of the discharge is similar to that found in the literature from [31], where the plasma is postulated to enter the streamer discharge state, compared to the luminescent stage which is referred to as a glow discharge.
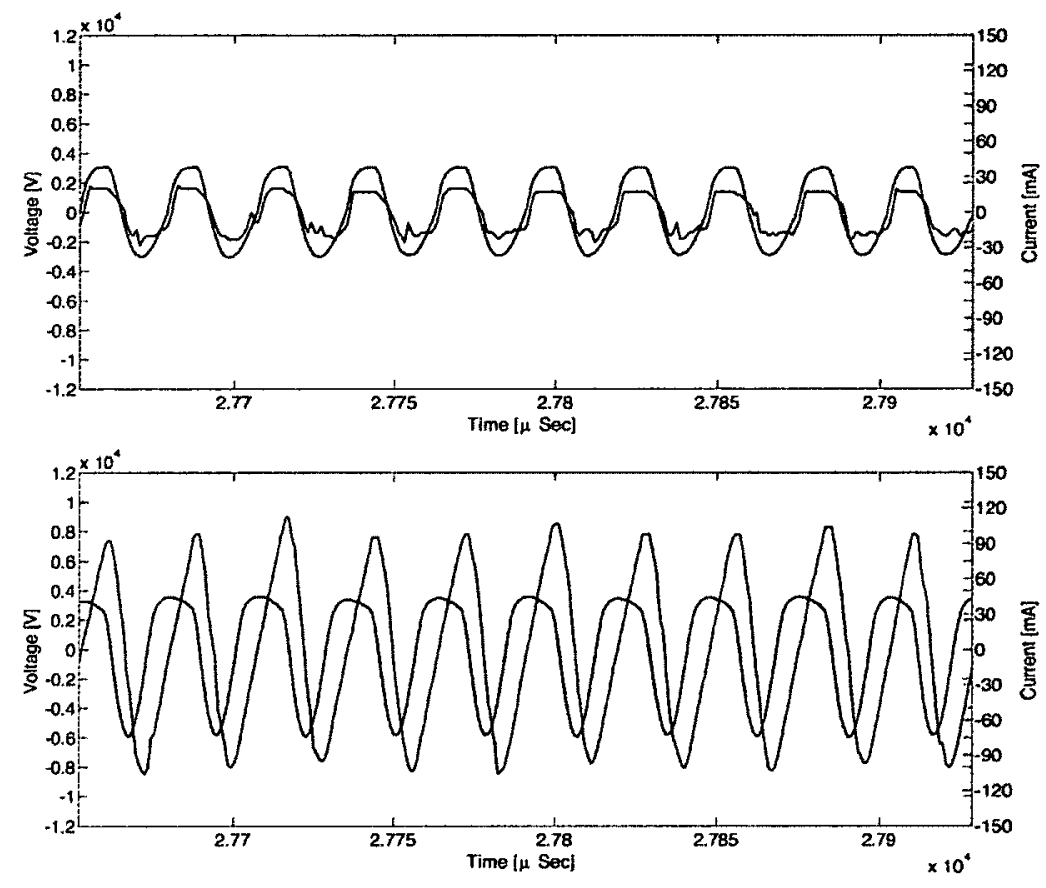

Figure 4.13: Enhanced image of voltage and current for both modes of the discharge seen in Figure 4.9 and 4.11. Spark profile for both (top and bottom) is; $\mathrm{BC}=$ $100, \mathrm{SL}=40, \mathrm{IL}=40, \mathrm{SP}=44, \mathrm{IP}=44$. 


\subsection{Conclusions}

In conclusion, the results presented here show that the plasma ignition device provides two plasma states as a function of pressure: at lower pressures it is a luminous discharge and at pressures above 110PSI it is a non-luminous discharge. This is also true for changes in current pulse width settings, where lower current settings provide the non-luminous plasma state at lower pressures compared to the higher current settings. This confirms that there exists a plasma cloud between the electrodes beyond the arc discharge of the SPDI unit, although two different types of plasma were seen. When compared to the conventional ID unit the SPDI discharges showed significant differences.

However, for cases inside a combustion chamber, the behaviour of the voltage and current curves are highly dependent on the local pressure and therefore resistance across the electrode gap. This is further depicted by the continuous transition between the two plasma states during a single spark event, Figure 2.7 of Section 2.3.1. The reasoning for this behaviour is postulated to be due to the flame kernel beginning its expansion after the arc discharge and thus transitioning the unburnt air/fuel mixture to an exhaust mixture. This results in a highly variable resistance across the electrode gap, thus affecting the plasma state exhibited. Further research into its validation is, however, reserved for another project. 


\section{Chapter 5}

\section{Internal Combustion Engine Experimental Methods}

\subsection{Experiment Apparatus}

The experiment conducted to evaluate the effects of a plasma assisted ignition system on the combustion process inside an internal combustion engine uses a single cylinder 6.5 horsepower engine connected in series to an electric generator. The apparatus, as seen in Figure 5.1, is equipped with the following instruments:

- pressure transducer;

- angular encoder;

- intake and exhaust thermocouples;

- barometer;

- ambient humidity and temperature; 
- SPDI system and conventional ID system.

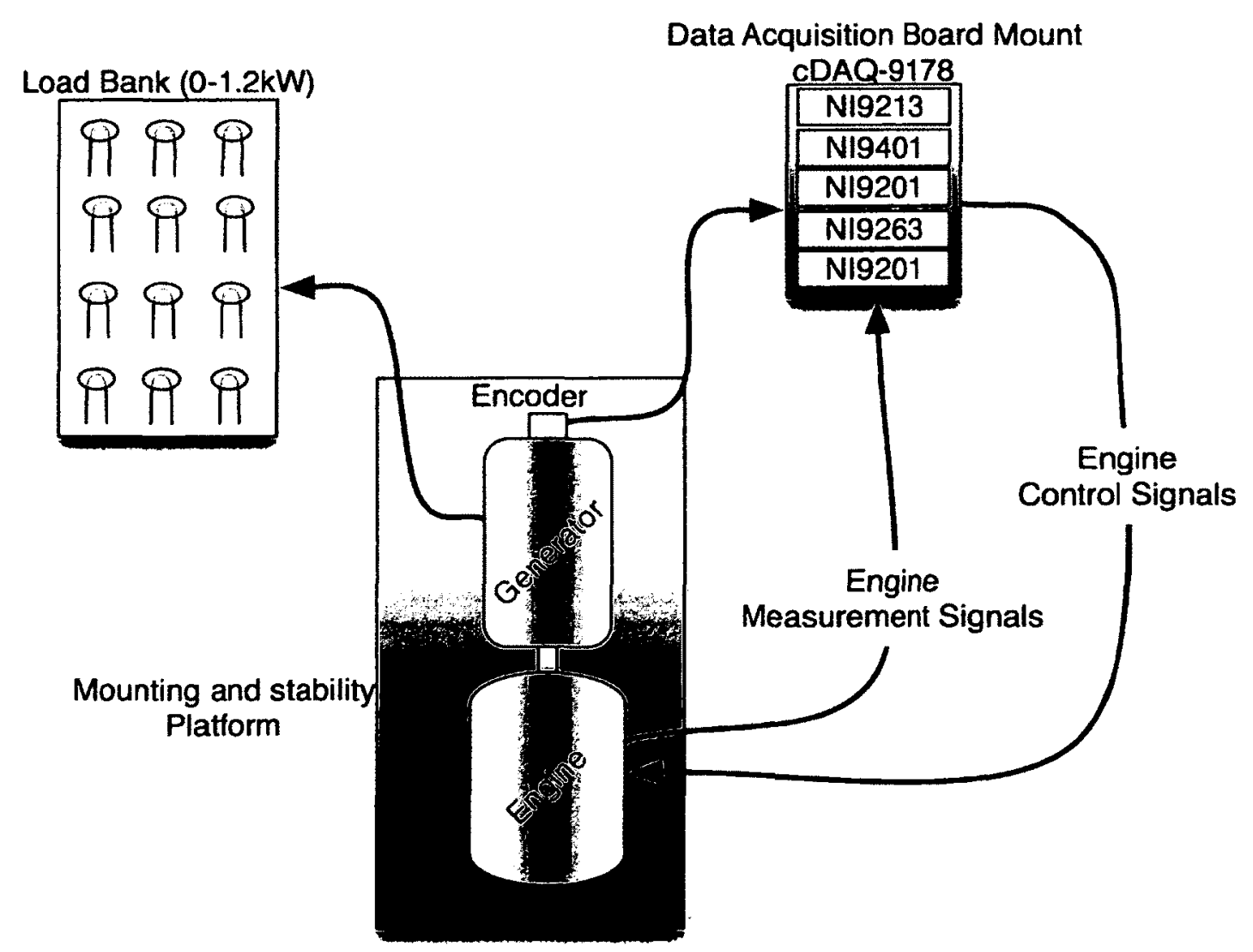

Figure 5.1: The generator setup and its respective components labelled.

The apparatus was wired according to Figure B.1, in Appendix B, and measurements were taken by a cDAQ-9178 equipped with two NI9201 modules, a NI9401 module, a NI9213 module and a NI9263 module.

The optical angular encoder makes use of a Transistor-Transistor Logic (TTL) signal that can be measured by high-speed rising edge activation counters equipped 
inside the National Instruments 9401 module and the 9178 module. These signals are then used for ignition timing as well as angular position measurements seen in section 5.1.1. The pressure transducer and thermocouples use analog signals which are measured by the analog input channels on the NI9201 modules and the NI9213 module. Details of the functionality and signal processing of each instrument can be found in the following sections.

\subsubsection{Engine Timing}

Engine timing is a critical component for this experiment's reliability. Through iterations of the system, a stable and controlled setup was established. The first iteration of timing control used a hall effect sensor, which had a fixed mounting angle at 20DBTDC. ${ }^{1}$ The system ran the engine, although for proper analysis of combustion, such as lean burn conditions, variable timing was necessary. This was attempted by developing a software based ignition timing device using the Arduino Uno hardware board.

It was speculated that a software based system would be able to control the ignition timing using the signal pulse frequency of an encoder wheel. Two different approaches were developed from this:

Approach 1 used a time-based system that counted the number of pulses in a given time period, derived the delay time for the appropriate ignition angle, and then outputted the signal to the ignition system. Due to the cyclic variability of the single cylinder engine this timing approach was not of adequate accuracy and was discontinued.

\footnotetext{
${ }^{1} 20$ DBTDC was chosen based on an approximation of the stock ignition system angular position.
} 
Approach 2 used an angular based method employing Arduino hardware and software interrupts to count the number of pulses and used this count to deliver the spark event at a designated angle. It was determined that the software was too slow and resulted in faulty timing of ignition triggers. However, the development process involved here established that an angular-based system was necessary for proper ignition timing.

Final Approach. Finally, we developed a satisfactory timing approach that used hardware for trigger activation but also software to control the timing of the trigger pulse and the pulse width. This system used an optical angular incremental encoder calibrated to produce 1000 pulses per revolution transistor-transistor logic (TTL) routed to the high speed counters inside the National Instruments data acquisition (NI DAQ) boards. The high speed counters on the NI DAQ boards were configured to output a $5 \mathrm{~V}$ pulse at a specific counter increment (which could be set through software manipulations). This solved the cyclic variability issue and allowed for faster response compared to software systems. The downfall of this system, however, was its susceptibility to noise, as will be discussed later in this section.

The angular encoder uses a three channel system (A, B and Z) where the B signal is phase shifted 90 degrees from $\mathrm{A}$ and the $\mathrm{Z}$ phase is used as the index which represents the completion of a single revolution. Figure 5.2 shows the system increment locations identified by 1's and 2's. This type of signal conversion is called $\mathrm{X} 2$ encoding. It is triggered on the rising and falling edges of a single channel. The $\mathrm{X} 4$ encoding uses the rising and falling edges of both signals. (Figure 5.3)

The use of $\mathrm{X} 2$ and $\mathrm{X} 4$ encoding allowed for angular increments of $0.18^{\circ}$ and $0.09^{\circ}$ respectively, compared to $0.36^{\circ}$ should only the rising edge of one channel be used 


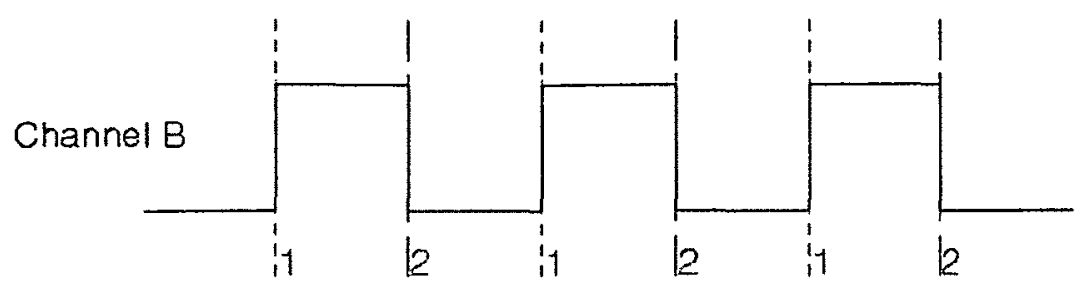

Figure 5.2: The two encoder signals contributing to incremental changes of the data acquisition counters using X2 encoding methods.

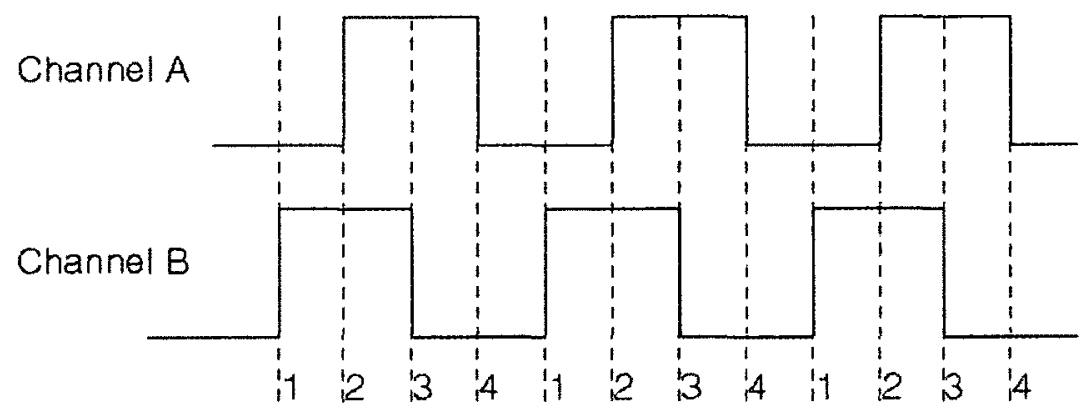

Figure 5.3: The two encoder signals contributing to incremental changes of the data acquisition counters using $\mathrm{X} 4$ encoding.

on a 1000 pulse per revolution encoder.

For the engine timing, the internal counters of the DAQ board use only one encoder channel, which was triggered by the rising or falling edge of the signal pulse. This provided the best resolution of $0.36^{\circ}$. As discussed earlier, the susceptibility of the counter to noise is illustrated in Figure 5.4. The signal acquired by the DAQ reacted to the rising edges at each point 1 , as well as the threshold breach by noise in the line, this caused the counter to rise one count and trigger the ignition system one counter position early.

Through post-processing techniques, the cycles in which the timing signal is falsely advanced can be omitted from the analysis since the angular position of the crank shaft is still known and faulty triggers can easily be identified. A better option 


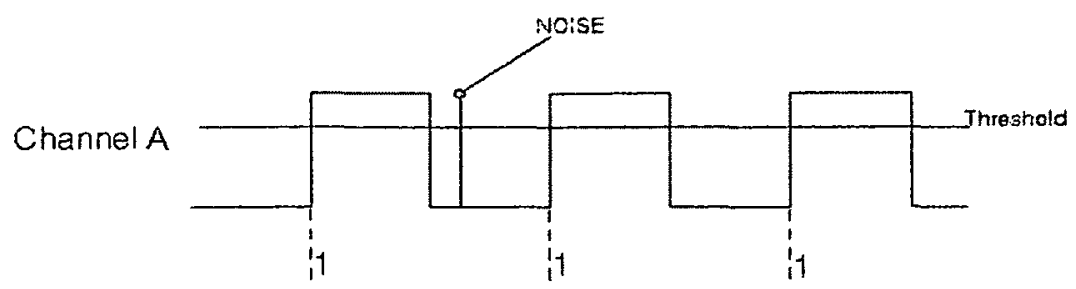

Figure 5.4: The effect of EMF noise overlapped on an encoder signal.

is to reduce the susceptibility of the high speed counters to noise. This can be done by increasing the amount of current flowing through the signal wires. One way to do this is to use a pull-down resistor between each signal wire and a grounding point (preferably near the DAQ boards). Such a schematic can be seen in Figure $5.5 .^{2}$

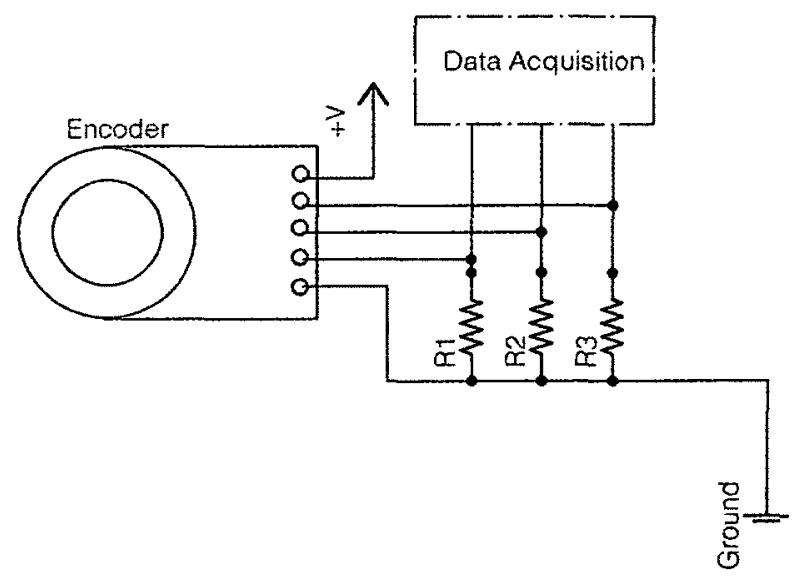

Figure 5.5: Schematic of an encoder wired with pull-down resistors.

A final point on this is to determine why the angular values recorded are not commonly increased by noise, this is because as the point 1 in Figure 5.3 is achieved the only sensible next position is either point $\mathscr{2}$ (which would represent an increment in angle) or point 4 (representing a reverse increment). Therefore, for noise to be

\footnotetext{
${ }^{2} \mathrm{It}$ is important to note that the current encoder can only source $8 \mathrm{~mA}$ of current. A more robust version would likely serve better in future research projects, allowing more current to flow and minimizing the effects of EMF noise.
} 
an issue it must have the proper timing to simulate the next sensible combination of true and false values between both encoder channels, or the change in voltage will be overlooked. If necessary, these cycles can be removed from the analysis through post-processing techniques.

\subsubsection{Air and Fuel Management}

The air and fuel delivery system consists of a mechanical carburetor, controlled by butterfly valves. While fuel injection systems provide more accurate measurements of fuel input, through pulse width modulation, this research required only an experiment which could reproduce consistent results amongst many trials. Therefore the simplicity and consistency of fuel delivery through the carburetor was chosen to be the most effective method. It was assumed that for a specific RPM and throttle position, the fuel delivery was constant. To ensure the quality of this assumption and the continuity of fuel delivery from trial to trial, the experiments were conducted on separate days and repeatability of the results was confirmed.

\subsubsection{Load}

The generator uses a load bank of light bulbs to provide a variable load to the engine. The operator monitors the voltage and current being released from the generator using a watt meter. Due to the cyclic variability of a single cylinder engine and the non-constant load of electric generation, the apparatus used here should be replaced with a constant-load fluid brake dynamometer when further investigations is performed. 


\subsubsection{Pressure Measurements}

The combustion chamber pressure measurements are gathered from an Optrand AutoPSI-S fibre optic pressure sensor installed in the head of the cylinder at the location outlined by the red circle in Figure 5.6. Details on the make and model of the unit are available in Appendix A.1. The choice to use an interferometric fibre optic pressure sensor, versus a piezoelectric crystal pressure sensor, was made based on availability and affordability. There exist research groups that have investigated the use of this pressure sensor in combustion environments ([32] and [33]) and the use of a spark plug mount, although this has proven to lead to some data deviations, when compared to a wall mounted piezoelectric pressure transducer, due to fluctuations of the mount during pressurization [32]. This is why it was chosen to mount the sensor directly into the wall of the cylinder head. Due to the thickness of the cylinder wall, the sensor head had to be mounted offset from the inner surface a distance of $6.35 \mathrm{~mm}$ or $1 / 4 "$. The sensor is pointed towards the centre of the combustion chamber such that the pressure variations inside the chamber are reflected more accurately by the sensors output compared to a transverse orientation (Figure 5.6). Specifications on the sensor and its signal conditioning unit are available in Appendix A.1.

\subsubsection{Ignition Coils}

The ignition coils seen in Appendix B.4 were used in conjunction with, alternately, the SPDI ignition system and the BOSCH ignition system. The coils, as provided by the manufacturer, have a linking connection between the primary and secondary 


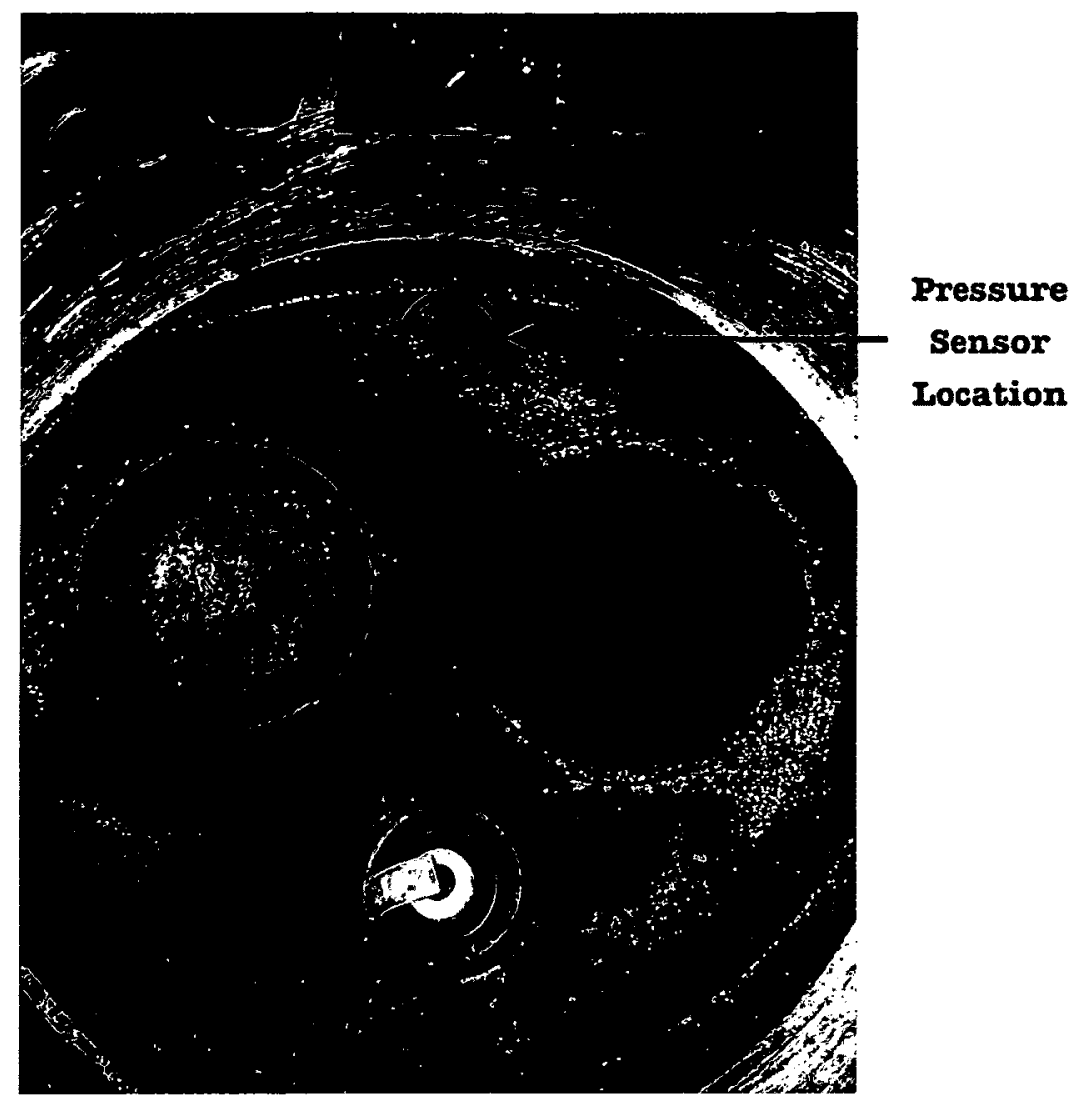

Figure 5.6: The installation location of the pressure sensor inside the cylinder head of the engine.

windings. This had to be removed such that the current flowing through the secondary coil could be measured directly (Figure 5.7).

From the measured voltage, Ohm's law is applied and the approximate current is deduced. The current measured here does not precisely reflect the current across the spark gap since other processes of ionization and electron emission are present, as discussed in Chapter 2. 


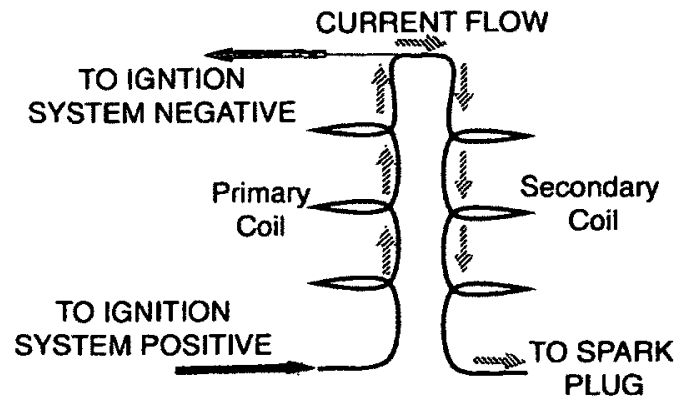

(a) Coils attached at the top with no access to secondary winding current.

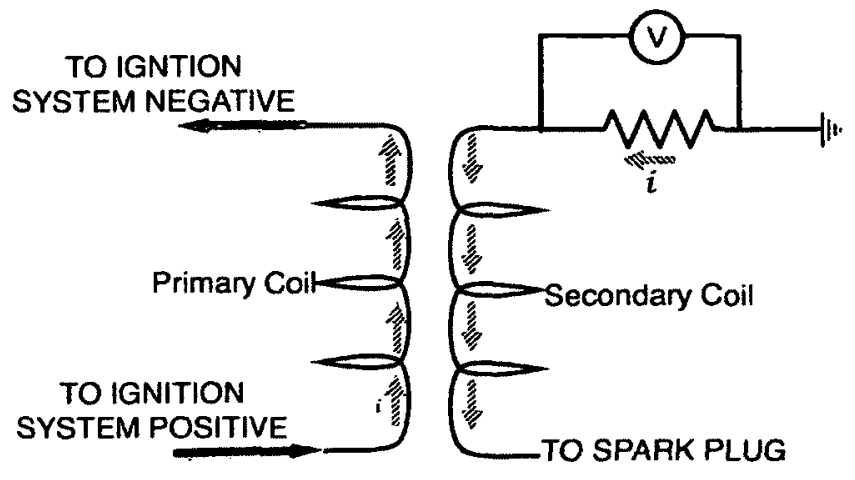

(b) Coils detached at the top with access to secondary winding current.

Figure 5.7: Adaptation of the ignition coils to monitor current through the spark plug.

\subsubsection{Oscilloscope Data Acquisition}

Measurement of ignition current and voltage was performed using a Link Instruments Inc. MSO-9201 oscilloscope connected as shown in the schematic in Figure 5.8 and Appendix B. The voltage measurement was performed by using a voltage step-down conversion unit with a conversion factor of 1150:1. This allowed the difference in voltage across the spark gap to be approximated. The current was deduced from the voltage measured across a resistor inline between the secondary coil of the ignition coil and a grounding point, as discussed in Subsection 5.1.5.

The MSO-9201 oscilloscope also includes an optional Logic Analyzer unit which was used to measure the pulse width of the ignition device. This signal was used as the trigger source for synchronizing the oscilloscope data with the NI DAQ system acquisitions. This allowed the analysis on a cycle by cycle period of the spark gap voltage, current and its resulting combustion pressure. Since the memory on-board 


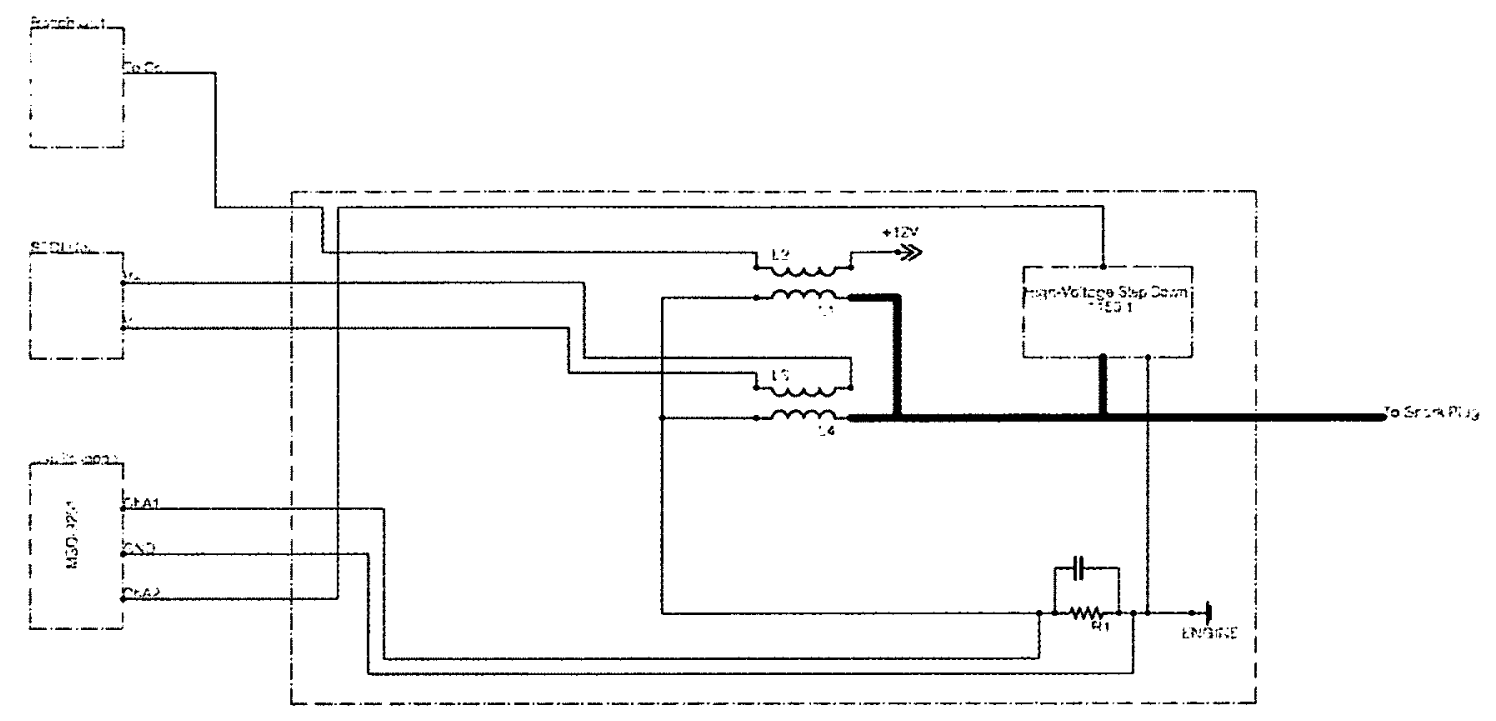

Figure 5.8: Schematic of coil arrangements for both SPDI and Bosch Units.

the oscilloscope was $512 \mathrm{~K}$, this allowed the capture of approximately 20 ignition cycles at a sampling rate of $500 \mathrm{KSa} / \mathrm{sec}$, or 4 ignition cycles at a sampling rate of $2 \mathrm{GSa} / \mathrm{sec}$.

\subsection{Motored Pressure Acquisition and Top Dead Centre Measurements}

This section will explain the process and methods involved in acquiring the motored pressure curve of the engine and the methods for approximating the location of top dead centre (TDC). 


\subsubsection{Motored Pressure Setup}

For engines equipped with multiple cylinders, motored pressure curves can easily be acquired by monitoring the cylinder pressure upon shutting off the ignition device in one cylinder, assuming there is enough torque from the remaining cylinders to maintain desired engine speed. For our experiment, the use of a single cylinder engine forced the use of an external rotational motor to turn the crankshaft. The electric motor choice was a PMG132 permanently excited direct-current motor operating at $48 \mathrm{~V}$ and power sourced from four $12 \mathrm{~V}$ deep-cycle lead acid batteries capable of 900 cranking amps. The disadvantage of the current setup is that it only allows the electric motor to travel in the reverse direction by reversing the polarity of the leads. This limits the rotational speed of the motor to 2870 revolutions per minute as specified by the manufacturer. The motor setup is seen in Figure 5.9, where the PMG132 is directly coupled to the gasoline engine through two Lovejoy Inc. BWLC (Bellows Clamp Style Coupling) couplers which can handle $1.0^{\circ}$ misalignment each. This allowed for the vibrational movement of the engine.

The NI9263 data acquisition board was used to control the Alltrax AXE7234 high-voltage controller such that the RPM of the engine could be set. During the acquisition trials, the throttle position and the RPM were varied. As seen in Figure 5.10, the obvious effect that throttle position has on motored pressure curves is substantial. A collection of curves were acquired and added to a database for further combustion pressure analysis, although, as previously discussed in Section 3.3 when sweeping across the TPS positions, the increments were too large and therefore lead to errors in the subtraction of the motored pressure from the cylinder pressure. 


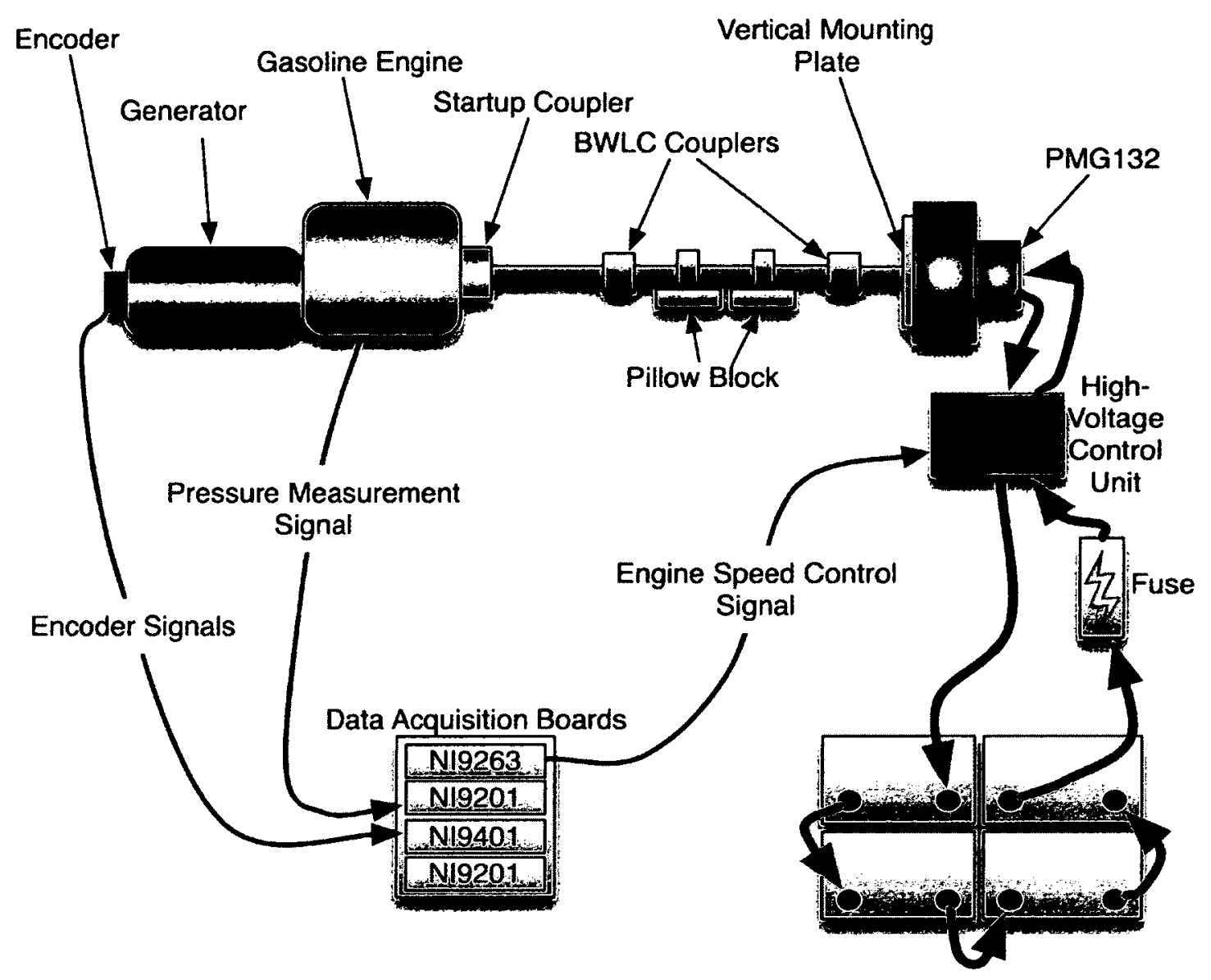

Figure 5.9: Setup for rotating the engine through the use of a PMG132 electric motor.

\subsubsection{Top Dead Centre Measurement Methods}

The definite position of TDC is critical for proper combustion analysis, but it is also difficult to measure. There have been numerous researchers looking into methods of determining TDC, such as Yamanaka et al. who have investigated the use of microwaves to measure TDC positions [34], or Tazerout et al. who have investigated 


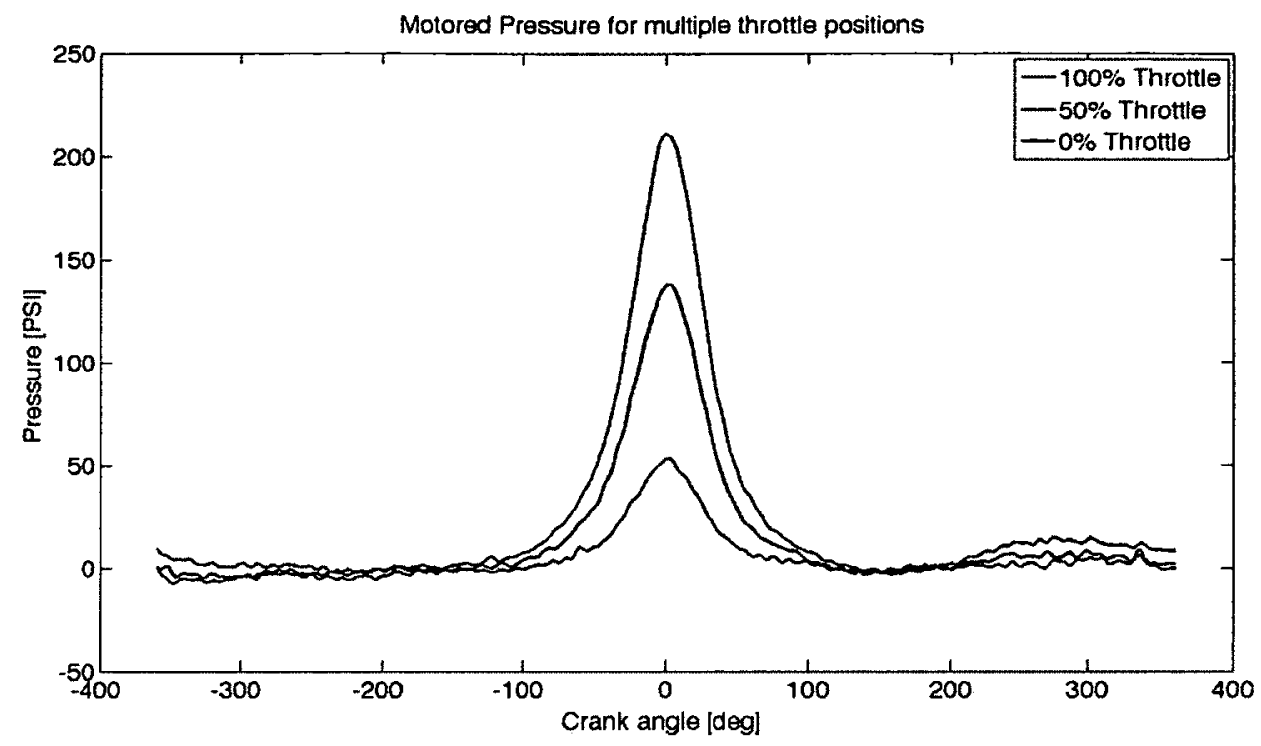

Figure 5.10: Motored pressure curves corresponding to throttle positions of $100 \%$, $50 \%$ and $0 \%$, all at 2870 RPM.

a thermodynamic approach [35]. The method of determining TDC in this apparatus made use of a linear potentiometer, outfitted with an axial spring, which was affixed to the cylinder head by clamps. The potentiometer was then placed through the spark plug opening perpendicular to the piston surface and the crank was rotated. To minimize deflections, a backstop was used behind the potentiometer. The produced voltage across the potentiometer was then read by the NI9201 data acquisition cards (sample curves are illustrated in Figure 5.11). The maximum travel of the potentiometer corresponded to the point at which the piston reached TDC. In the enhanced portion of Figure 5.11, there are three arrows, the outer extremity arrows being the limits of when the slope of the curve reaches zero and leaves zero. This midpoint is then calculated and taken to be the TDC of the stroke. This is subsequently reproduced for every cycle and an average value for TDC is calculated. 


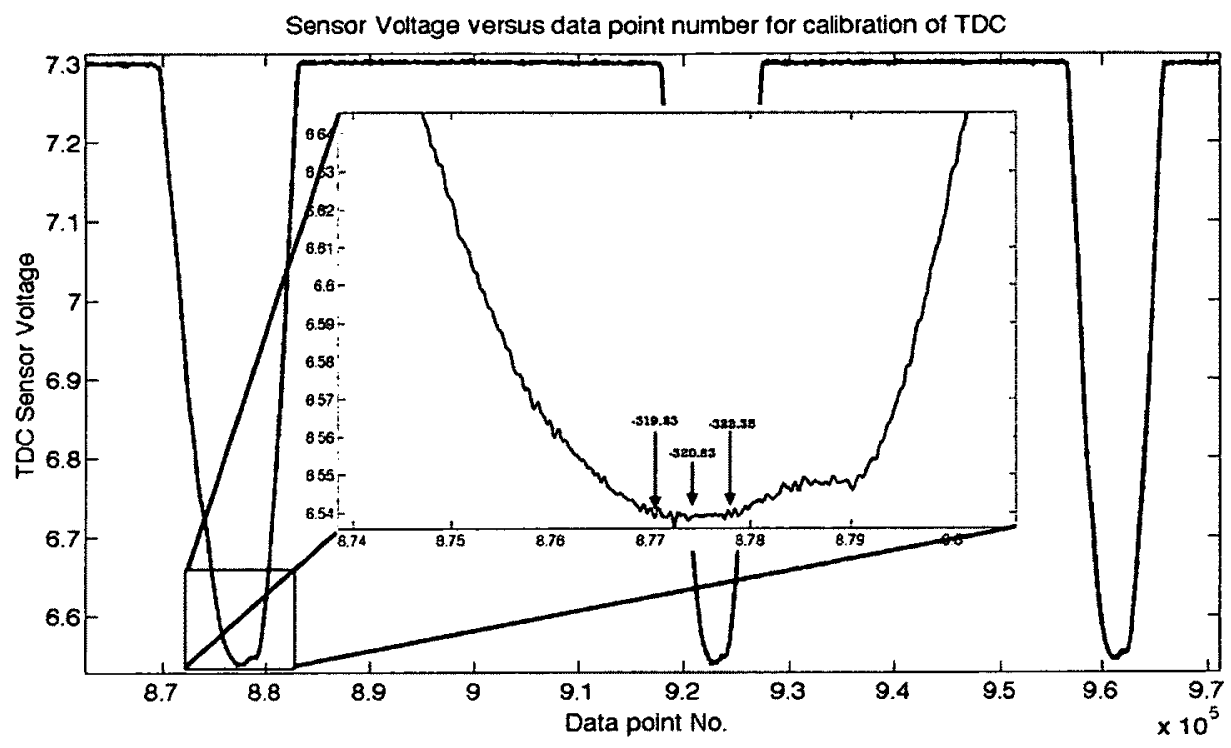

Figure 5.11: TDC sensor voltage curves corresponding to three strokes of the engine. In the enhanced section, three arrows are drawn, the outers corresponding to the locations at which the switch in piston direction first occurs and therefore the midpoint would correspond to the location of TDC.

The dynamic TDC is not necessarily equivalent to that of the static measurements due to crankshaft twist and non linear compression of the gaseous mixture inside the combustion chamber. It was assumed in this research project that they were equivalent. A real-time approximation of TDC would be required to provide further insight into the variability of TDC location for each cycle. This system could use a reference signal such as a hall effect sensor affixed to the engine to provide a stationary reference while the engine is in use to provide insight into crank shaft twist. 


\subsection{Other Acquisition Techniques and Procedures}

Noise reduction and shielding are crucial for proper signal acquisition and analysis. This section will outline some of the techniques used for isolating ground loops, reducing EMF noise and reducing digital/analog switching noise.

\subsubsection{Acquisition of Signals and Noise Reduction}

As discussed earlier in section 5.1, the data acquisition system used to monitor and control the engine is a National Instruments cDAQ-9178 equipped with two NI9201 modules for analog inputs, a NI9401 module for digital line input and output, a NI9213 for thermocouple voltage measurement and a NI9263 module for analog output signals. The routing of each terminal of every module is illustrated in Appendix B.1 where the wiring schematic of the sensors, engine and DAQ ports have been identified.

Two factors became important during the development of wiring: ground loop isolation and power supply choice. Ground loop isolation is a systematic approach to reducing noise. It was determined that the simplest method of identifying loops was to have all grounding points mount to a single location on the engine.

As for the choice of power supplies, it was determined to be as important as removing ground loops. The signals were analyzed using the Fourier Transform analysis and it was determined that the frequencies of the noise were multiples of the switching frequency of the power supply. This led to the requirement for a nonswitching power supply. The final result is the wiring schematics seen in Appendix B. This modification provided the adequate $12 \mathrm{~V}, 10 \mathrm{~V}$, and $5 \mathrm{~V}$ power supplies for all 
sensors and ignition units.

\subsubsection{Signal Shielding}

In high-voltage and high-current applications, signal wire shielding is extremely important and should not be overlooked. This is because the electrical and magnetic fields produced by the high-voltage wires can cause undesirable voltage spikes in the signal wires of other instruments. To prevent this noise from entering the low-voltage wires, the digital switching signals from the encoder were conducted through a five wire aluminum and Beldfoil shield twisted wire where the shields were single-end grounded. The analog voltage signals from the pressure transducer, the throttle position sensor and the air-to-fuel ratio meter used a Belden single-pair cable also consisting of aluminum and Beldfoil shields, with single-end grounding. This provided sufficient isolation from noise to acquire quality signals.

\subsection{Systematic Error and Instrument Hysteresis}

With all experimental apparatuses, there exists inevitable systematic error from the instrumentation. This section will discuss some of the systematic errors and random errors that were discerned from our instruments.

Since the Optrand AutoPSI-S interferometric fibre optic pressure transducer was the largest contributor to the data analyzed, it is the first topic of discussion. According to the manufacturer the non-linearity and hysteresis error is combined to show $\pm 1-3 \%$ of Full Scale Operation (FSO) error during operation with combustion. This however is below the postulated effect that the ignition system is proposed 
to invoke on the resulting engine performance $(4 \%-9 \%)$, by the manufacturer.

The second instrument under investigation for causing systematic error is the optical angular encoder. According to the manufacturer, the maximum error that can occur per revolution is 5.5 degrees of rotational error, although typically only 3.0 degrees of rotational error is seen. These deviations from the true value should not be overlooked when discussing resulting crank angle locations for specific combustion characteristics (e.g. MFB 50\% location, EOC location, etc.).

the pressure plots seen in the results of this thesis inevitably include these errors as well as the statistical errors developed from cyclic variability due to the engine setup. It is therefore concluded that the 1-3\% error resulting from the pressure transducer is not outside the $4 \%$ to $9 \%$ expected difference upon changing the ignition system. As well, the 3-5.5 degrees of rotational error from the encoder is well below the standard deviations of the MFB calculations. 


\section{Chapter 6}

\section{Post-Processing Techniques}

Post-processing of the signals required the creation of a new program designed specifically for the parameters measured in this experiment. Two programming software tools were used; LabVIEW and MATLAB, each providing their own benefits. This chapter will discuss the following programs;

- a LabVIEW program to control the engine and acquire raw data;

- a MATLAB program to analyze the signals and create a database of pressure and mass fraction burn curves;

- a LabVIEW program that combines the engine control program and the signal analysis to create a real-time acquisition and analysis tool. 


\subsection{Engine Control Program and Initial Acquisi- tion}

The engine control program configures the National Instruments hardware for engine timing, and also monitors the engine parameters. Many iterations of this program have been created due to a sequence of arising issues, such as counter configuration, acquisition synchronization and display iterations. In the interest of future students continuing the work, this portion of the thesis will outline some of the difficulties overcome as well as an outline of the software.

\subsubsection{Engine Timing Configuration}

The software configures the hardware so that three internal high speed counters monitor the encoder signals, one of which determines the angular position and returns these values to the software (ctr0). The second (ctr1) counts the number of encoder pulses and outputs a digital true signal to the third counter (ctr3) which then outputs only a single pulse for whichever duration is specified by the user. This method of using three separate counters allows for pulse width modulation (PWM) as well as pulse width timing (PWT). This is necessary since the inductive discharge unit uses the pulse width to determine the amount of energy stored in the ignition coil prior to discharge. 


\subsubsection{Sampling Rates and Synchronization}

The software configures the hardware to use the same sample clock across all channels, including the analog voltage channels, at $100 \mathrm{kHz}$ sampling rate. However, assigning the same sample clock is not adequate, all channels must also be triggered simultaneously to ensure synchronization ${ }^{1}$. The start trigger for sampling is the index channel of the encoder, this is a "non-retriggerable" event, which means it will only trigger the start of acquisition once, thereby synchronizing all channels. For the engine timing, the encoder index is used as a retriggerable event, allowing the reset of the ctr 1 and $\operatorname{ctr} 3$ counters.

\subsubsection{Acquisition}

The program is set up to enter two simultaneous while ${ }^{2}$ loops, one for controlling the engine timing and the other for acquiring data. The engine timing parameters, such as number of ticks counted per cycle, are updated every 10 milliseconds allowing us to set the advancement or retarding of the engine. The second while loop consists of a five-partition state machine that determines the user interface and is defined by the following.

- User: This state provides a graphical user interface (GUI) which analyzes the incoming signals and provides the user with averaged parameters such as engine exhaust temperature (EGT), intake temperature (IT), throttle position (TPS),

\footnotetext{
${ }^{1}$ The synchronization detailed here does not however take into account signal delay incurred by its travel along the length of the wires, it pertains to the hardware configuration inside the data acquisition cards only.

${ }^{2} \mathrm{~A}$ while loop is a software design command used to iterate a set of subcommands until a condition is met and the loop is exited
} 
engine speed (RPM), etc.

- Acquire: This state sets the number of samples based on a predetermined amount from the user and acquires the data (typically one million samples/channel) from all channels, it then saves all the raw data in text files. The data is further analyzed by the MATLAB program described later.

- Graphical Display: This state is for debugging. All the signals are left as raw data and displayed on the front panel for the user to observe.

- Analyze: This state is the real-time analysis of the engine data, further discussion into methods used is available in Section 6.3.

- Stop: This state shuts down the engine and data acquisition in a sequential order such that the data acquisition boards do not continue to output signals such as the ignition trigger signal. If not implemented this could result in the destruction of the ignition unit and coil.

\subsection{MATLAB Post-Processing Techniques}

Once the signals are acquired by the LabVIEW control program, post-processing is performed in MATLAB using the program techniques discussed here. A flow chart of the program procedures can be found in Appendix C.1.

\subsubsection{Filtering and Signal Analysis}

Through techniques discussed in Section 5.3, the signal noise was reduced. However, to further reduce the noise a Butterworth low-pass filter design was implemented 
as per Equation 29. The filter coefficients $a$ and $b$ are designed by the MATLAB butter.m function with filter order $n$. The filter coefficients are then implemented by the MATLAB filtfilt.m function which is a zero-phase digital filter, as:

$$
H(x)=\frac{b_{1}+b_{2} x^{-1}+b_{3} x^{-1}+\ldots+b_{n+1} x^{-n}}{1+a_{2} x^{-1}+a_{3} x^{-1}+\ldots+a_{n+1} x^{-n}}
$$

The result of using this filter design is seen in Figure 6.1, where a single 4-stroke acquisition is plotted as the raw data and the filtered data. The residuals of the filtered data is available in Appendix C.4.

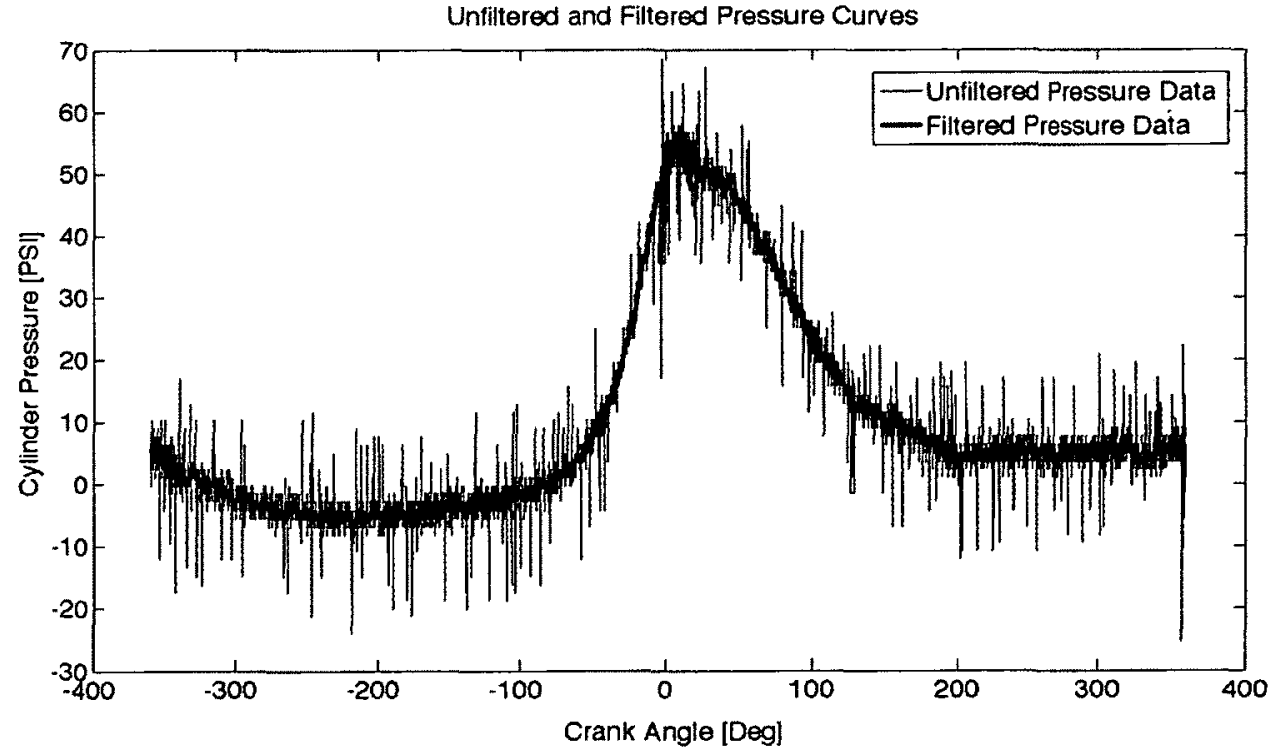

Figure 6.1: A plot of the filtered pressure overlapped over the non-filtered pressure curve.

The final analysis component of the MATLAB program uses the Mass Fraction Burn (MFB) analysis outlined in Section 3.3. This estimates the amount of fuel burnt from the pressure rise in the cylinder. The results of these curves are then fitted 
according to a Wiebe function with variable parameters and the results are saved. Since the evaluation of maximum MFB rate is calculated from the Wiebe function, the effects of noise on the signal and resulting noise on the slope calculations are reduced.

\subsubsection{Program Process}

The MATLAB program is structured according to the chart in Appendix C.1. The process includes the following:

1. import the raw data files from the LabVIEW program;

2. convert the data appropriately into the correct units;

3. proceed through a series of saving and importing of individual pressure curves;

4. write the mean, mode, and median pressure curves to a text file;

5. conduct the MFB analyses and write the results to a text file;

6. write a resulting XML file that specifies the parameters of each acquisition.

At this point, the statistical data curves are saved and the MFB curves are saved. The Database GUI is then used to quickly access the curves and their associated data from the XML files. The stored data in the XML files include parameters such as: ignition system type, SPDI discharge parameters, maximum slopes of each curve, maximum pressure, as well as the mean, mode, median and standard deviation of: load, throttle position sensor (TPS), exhaust gas temperature (EGT) and intake temperature (IT). This program allows for more efficient analysis of the data by 
having it organized and readily available. A front panel view of the Database GUI is available in Appendix C.2 along with an outline of its functionality.

\subsection{Real-Time LabVIEW Engine Control, Acqui- sition and Performance Analysis}

The use of the MATLAB program is excellent for post-processing data, although a more efficient method is to analyze data immediately following the acquisition. Therefore, with the knowledge gained from hardware configuration in LabVIEW, another program was written which enabled the use of real-time data acquisition and analysis. The concepts and techniques used in the MATLAB program (i.e. filter design, slope approximation, etc.) were translated into LabVIEW code then implemented in the engine control program. The real-time analysis program allows for the following parameters to be calculated and displayed immediately following an acquisition:

- mean, median and mode pressure curves;

- mean TPS, ET, IT and Load;

- combustion pressure rise;

- mass fraction burn curve;

- pressure versus volume curve;

- histogram of maximum pressure rise slopes; 
- histogram of maximum mass fraction burn slopes;

- histogram of maximum pressures;

- identification of crank angle at 50\% MFB.

\subsection{Statistical Analysis of Curves}

With engine data analysis, statistical approaches are recommended in order to account for inherent variability in engine cycles. This section will outline the steps taken from data collection to the data seen in the results and discussion portion of this thesis.

Plotting the pressure versus crank angle shows significant cyclic deviations (Figure 6.2).

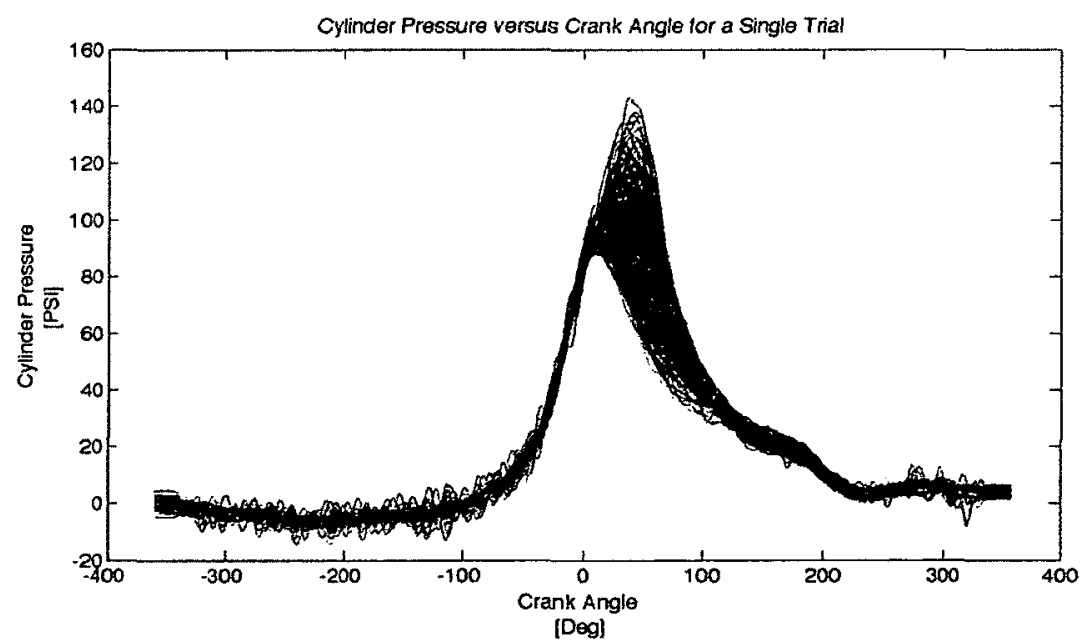

Figure 6.2: Cylinder pressure versus crank angle for a single dataset trial which consists of five different sessions under the same engine conditions. The total number of curves is 1071, where each session had approximately 250-260 individual cycles. 
The mean, median and mode of the data are tools that can provide a quick analysis of the dataset (Figure 6.3) ${ }^{3}$. When the mean of the curve is evaluated from a dataset the standard deviation must also be evaluated, for this example a plot of the mean and its standard deviation is illustrated in Figure 6.4. Where the solid line represents the mean of the pressure traces at each crank angle, while the upper dashed line $(-\cdots)$ represents the standard deviation added to the mean at its respective crank angle, the lower dashed line (_ - - ) represents the mean with the subtraction of the standard deviation at its respective crank angle. With this in mind, the figure also illustrates that the maximum standard deviation corresponds to $15.6 \%$ of the mean.

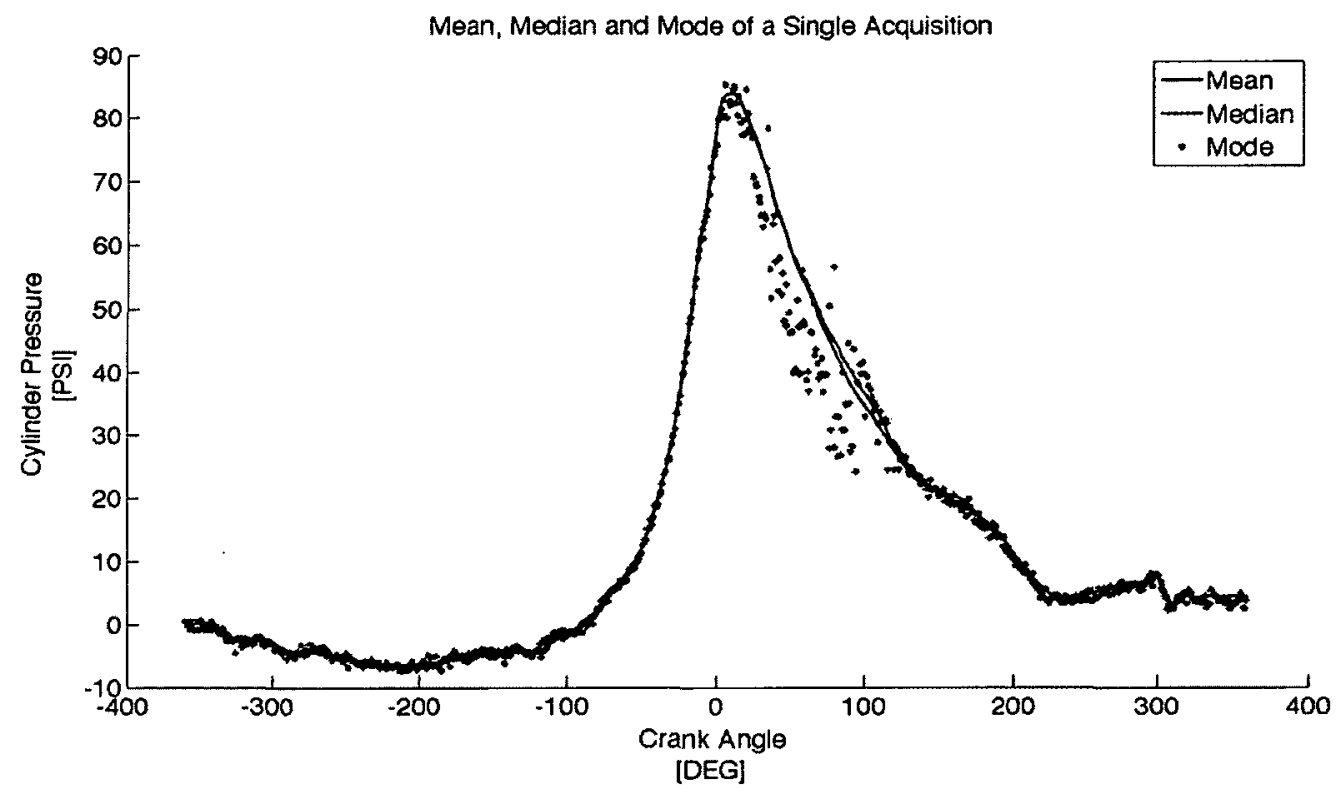

Figure 6.3: Mean, median and mode of cylinder pressure versus crank angle for a single dataset.

\footnotetext{
${ }^{3}$ The equivalent curve in the format of cylinder pressure versus volume is available in Appendix C.5, Figure C.5.
} 


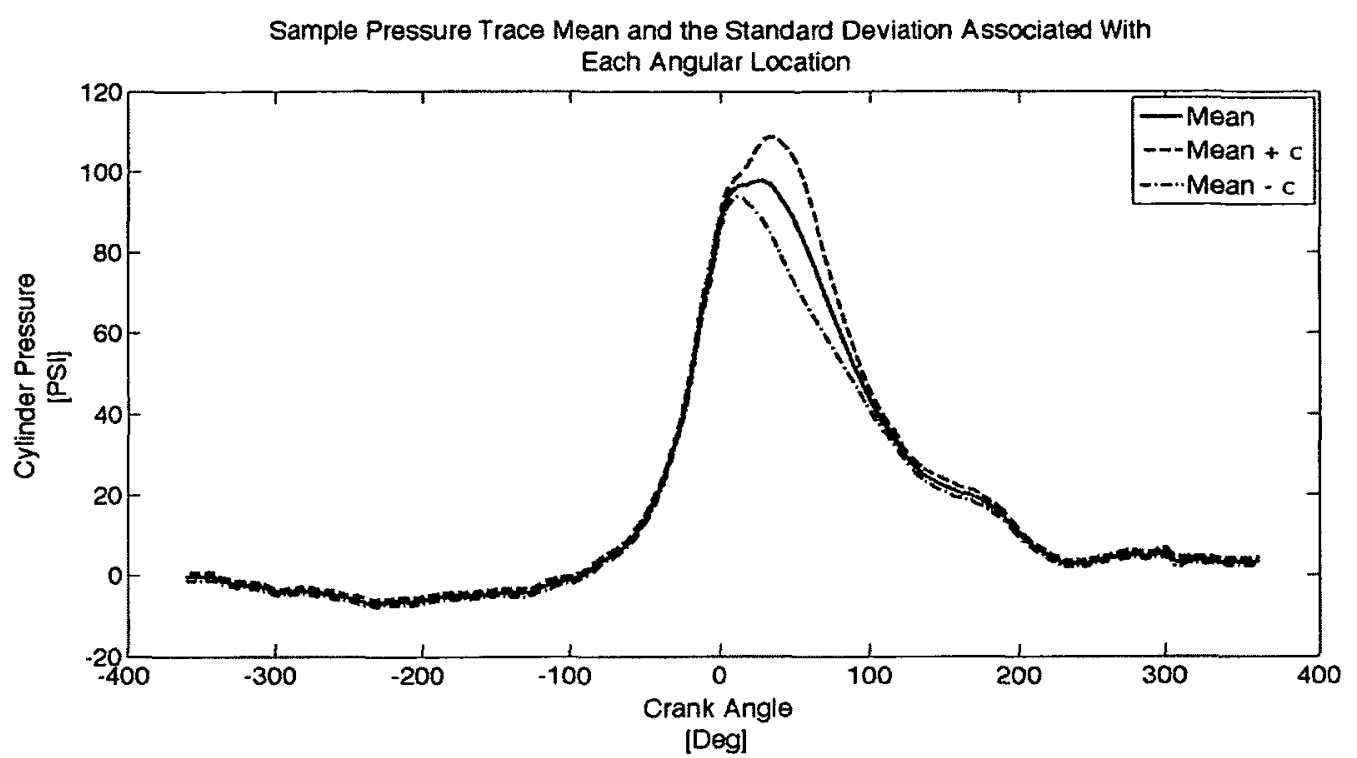

Figure 6.4: Mean cylinder pressure and standard deviation, versus crank angle for a single dataset.

In an effort to minimize the standard deviation of the data, 4 to 5 acquisitions consisting of approximately 250-260 ignition cycles were taken. ${ }^{4}$ This increased the number of samples per dataset. The standard deviation $(\sigma)$ is calculated as:

$$
\sigma=\sqrt{\left(\frac{1}{n-1} \sum_{i=1}^{n}\left(x_{i}-\bar{x}\right)^{2}\right)}
$$

where $n$ is the number of samples, $x_{i}$ is a point in the dataset $(i=1,2,3, \ldots, n)$ and $\bar{x}$ is the mean of the data. This equation highlights the fact that we can lower the sample standard deviation by increasing the sample size, $n$. However, this will only work well if the data we are sampling is in fact Gaussian in nature, which was no the case for all engine loads. Taking a closer look at the data revealed this non-gaussian distribution,

\footnotetext{
${ }^{4}$ All acquisitions were made on a time-base system consisting of $1,000,000$ samples/channel at a rate of $100 \mathrm{kHz}$.
} 
and hence was the reason for a large standard deviation. The result for a specific point in the pressure crank-angle curve (180DBTDC, end of intake stroke) can be seen in (Figure 6.5). The normal plot shows that the linearity of the data near the maximum probability is in agreement with the ideal normal distribution curve. This is also true for locations in the exhaust stroke (Figure C.4 of Appendix C.5). This is expected since the cyclic deviations during the intake, compression and exhaust strokes should not vary significantly.
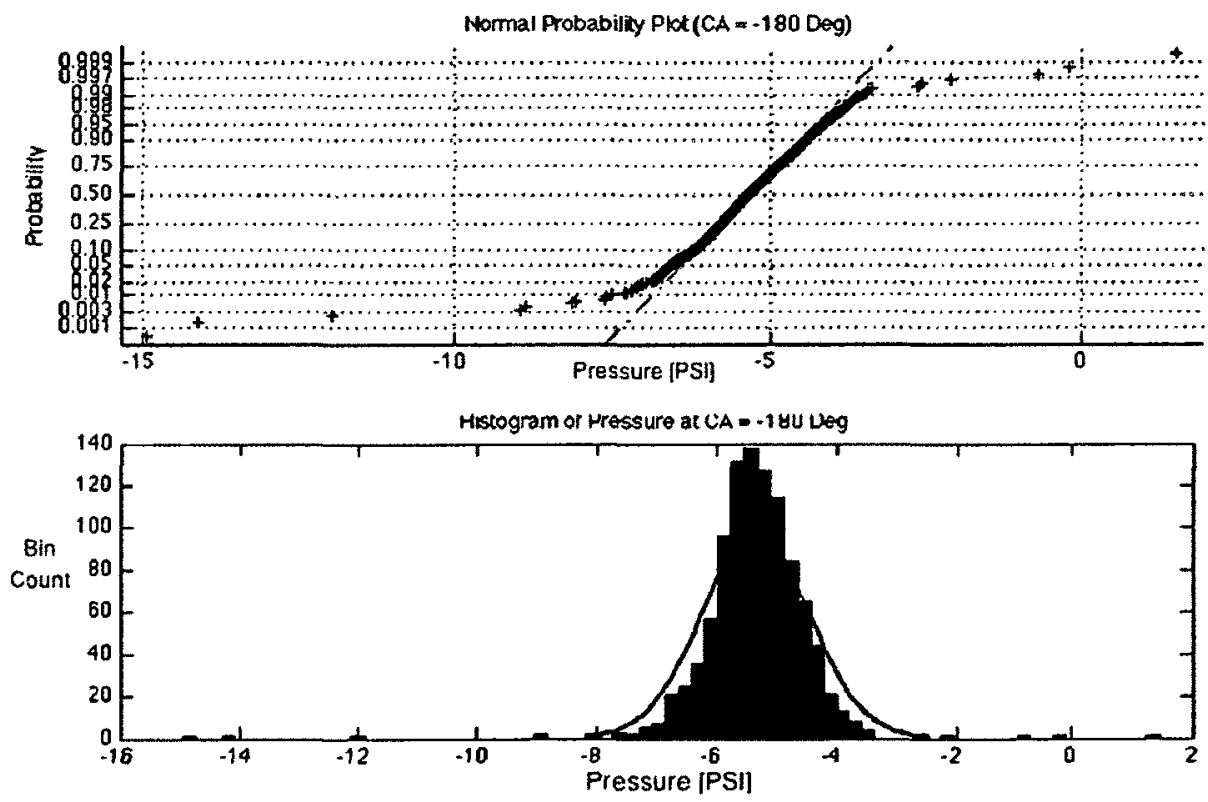

Figure 6.5: Histograms and normal probability plot for a single trials cylinder pressures corresponding to 180 DBTDC crank angle location.

However conducting the same exercise for the locations beyond TDC and before the exhaust stroke (Figure 6.6) leads to a skewed distribution. This is further observed by the non-linearity of the normal plot between 86 PSI and 89 PSI and at positions beyond 99PSI (Figure 6.6) where the blue markers separate from the scaled 
ideal normal distribution curve (i.e. red-dashed line). As discussed earlier, one of the reasons for the large standard deviation when taking the mean of the pressure traces can be explained by the lack of normality of the data points at each crank angle during the expansion stroke. In short, the assumption of a Gaussian distribution is not valid for points after top dead centre at some engine loads.
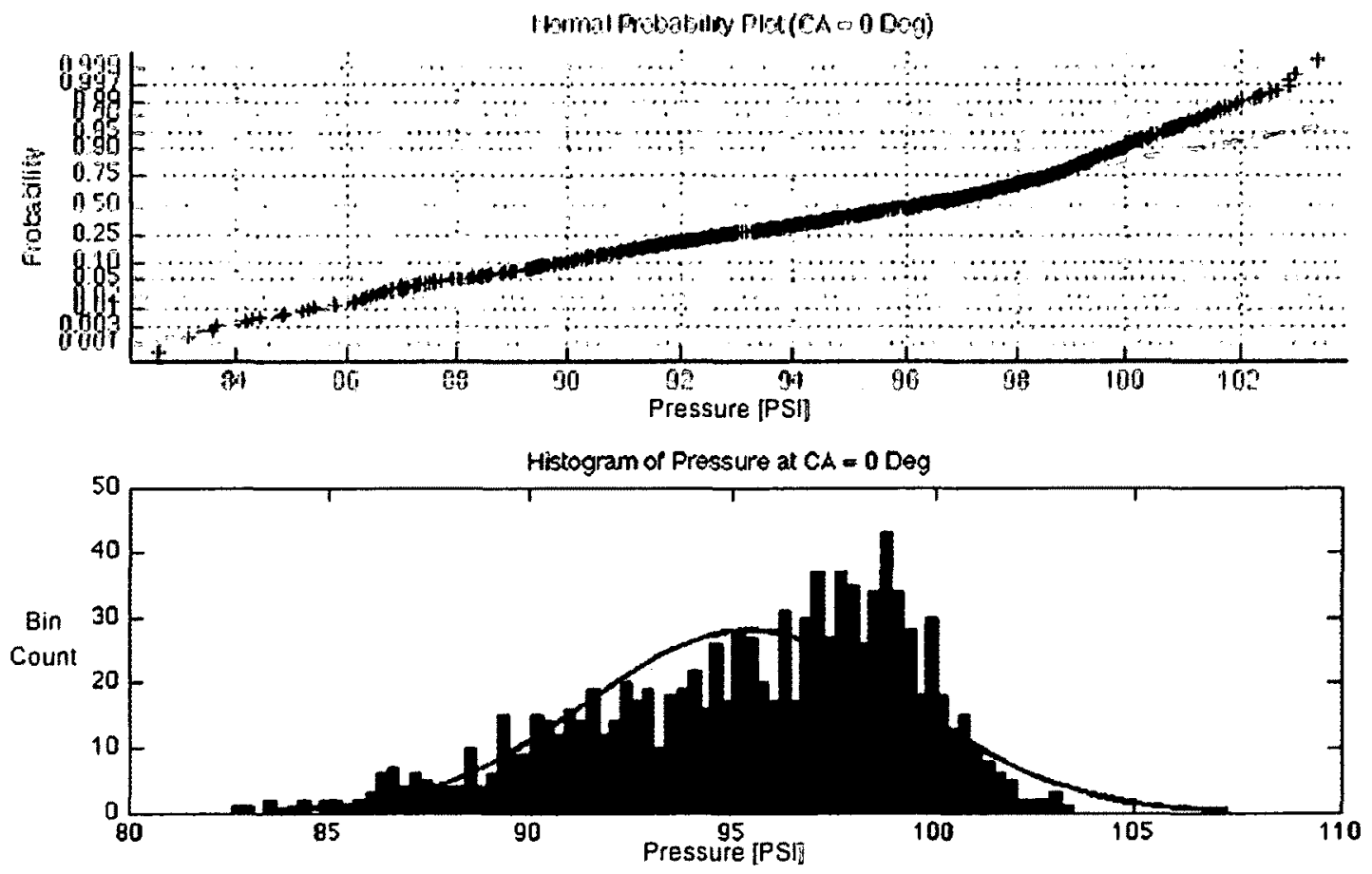

Figure 6.6: Histograms and normal probability plot for a single trials cylinder pressures corresponding to 0 degrees crank angle location (TDC).

Based on these observations, the MFB analyses were also examined in light of a skewed distribution. Plots for the crank angles corresponding to $M F B=0.5$ for the three engine loads $345 \mathrm{~W}, 755 \mathrm{~W}$, and $1180 \mathrm{~W}$, are seen in Figure 6.7. It is clear that there exists a transition in the distributions dependent on engine load and that 
the cyclic variability was highest at a medium engine load $(755 \mathrm{~W})$. However, it was also seen that variability between sessions existed for low engine loads (355W), this can be attributed to a number of reasons since small fluctuations in parameters at low engine loads can cause drastic changes in the engines behaviour. Therefore, this thesis will focus on presenting the results at an engine load equivalent to $1180 \mathrm{Watts}$ or approximately $60 \%$ of maximum engine load. Equivalently, since at higher engine loads the distributions are considerably closer to a normal shape, the results can be approximated by a mean curve. In addition, due to the histogram shape having an important role on the results, the histograms will therefore be included outlining the following criteria: crank angle corresponding to $50 \% \mathrm{MFB}$, rate of MFB change at $\mathrm{MFB}=0.5$, and the crank angle corresponding to the EOC location. An example is shown in Figure 6.8.
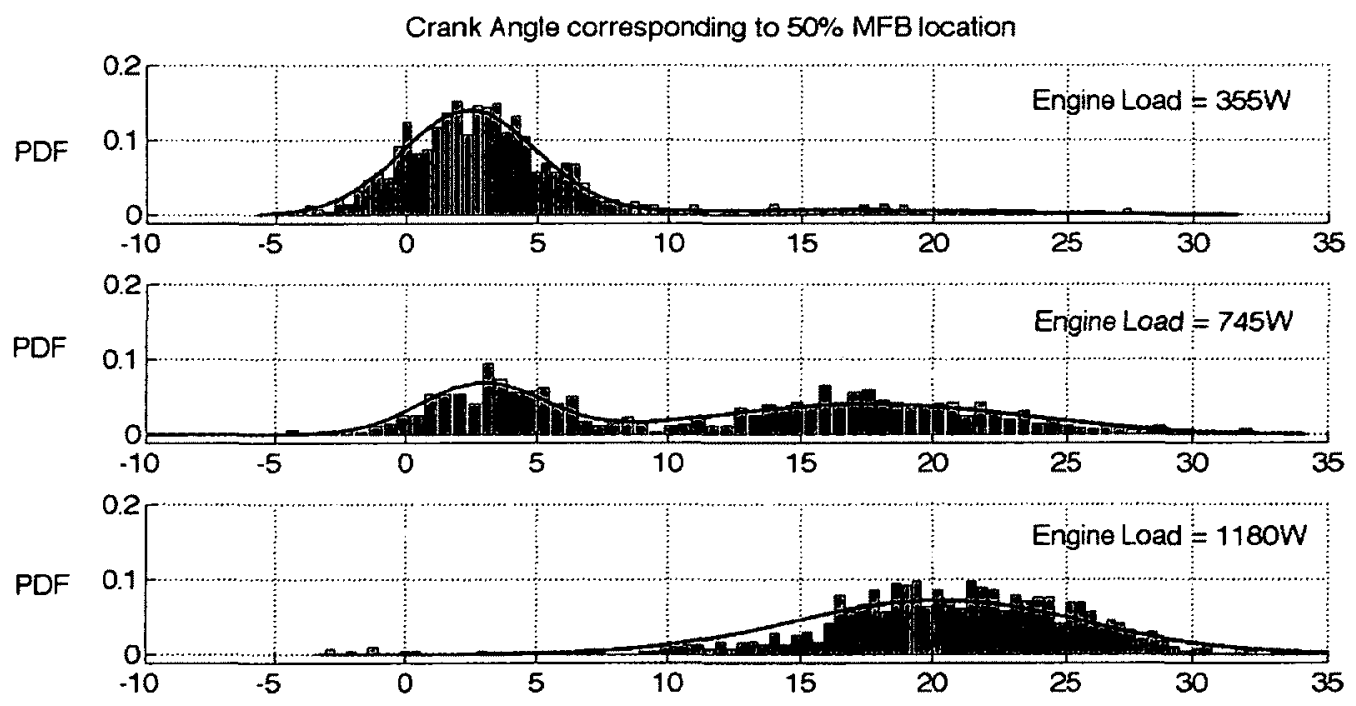

Figure 6.7: Histograms of crank angle corresponding to $\mathrm{MFB}=0.5$ for three different SPDI trials with the same spark profile and different engine loads, $355 \mathrm{~W}, 745 \mathrm{~W}$, and $1180 \mathrm{~W}$. 


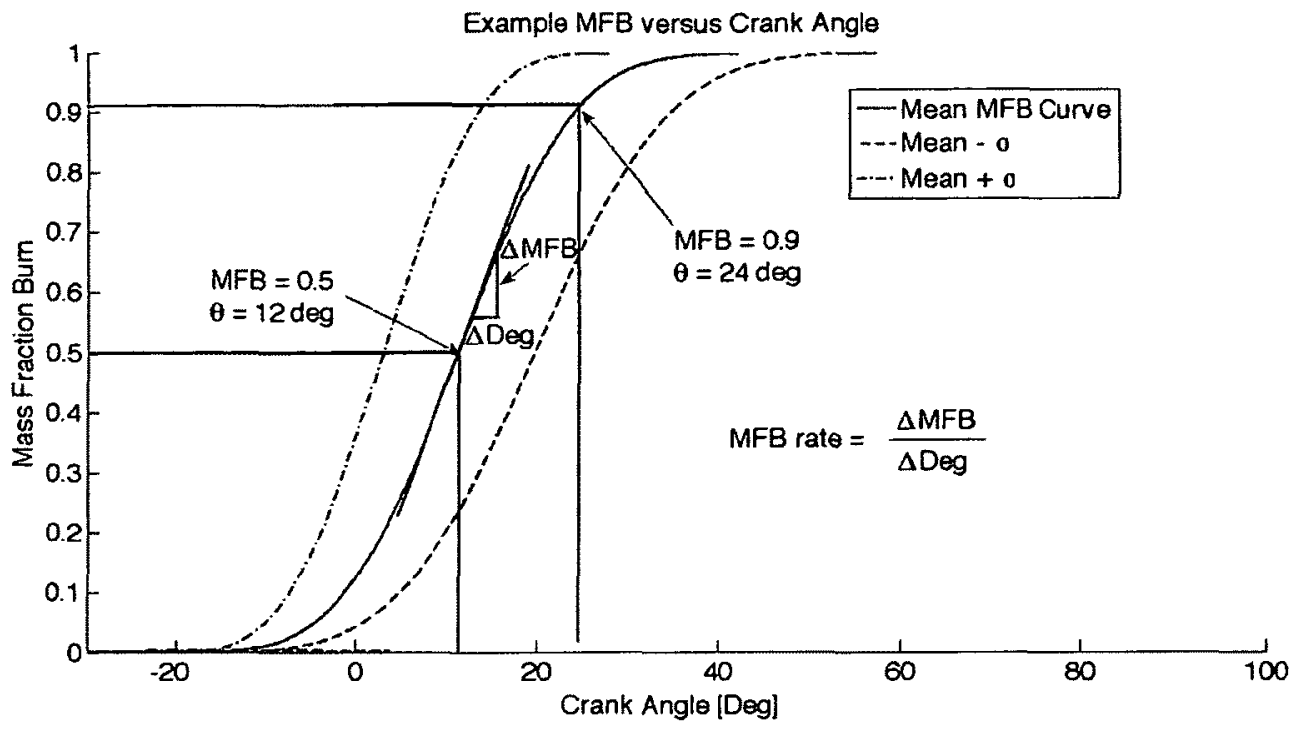

Figure 6.8: Sample plot of MFB versus Crank Angle for a trial at $755 \mathrm{~W}$ engine load. The parameters used in the production of the histograms include $M F B=0.5$ location, $\mathrm{MFB}$ rate at $\mathrm{MFB}=0.5$ location, and $\mathrm{MFB} \mathrm{EOC}$ corresponding to $\mathrm{MFB}=0.9$. 


\section{Chapter 7}

\section{Results and Discussion of Internal Combustion Engine Pressure}

\section{Measurements}

The experiment measuring the pressure rise rates inside a combustion chamber of the single cylinder engine made use of two ignition systems, one inductive discharge unit and a SPDI (Spatial Plasma Discharge) unit. This chapter of the thesis will outline the conclusions of the experiments comparing the resulting cylinder pressure due to changes in the SPDI ignition device plasma parameters, as described in section 2.3 and then comparing the effects of exchanging the SPDI device for the inductive discharge unit. Furthermore, an MFB analysis is performed on the pressure traces and conclusions regarding combustion characteristics are made. This experiment was developed in an effort to validate or invalidate the industrial partners claim that a $4 \%$ to $9 \%$ increase in engine performance is possible through use of the SPDI ignition device compared to a conventional inductive discharge (ID) device. As was decided in Section 6.4, for engine loads below $60 \%$ of maximum, the engine instability and 
resulting cyclic variability precluded statistically significant results. Therefore, the analyses shown here will only use the $60 \%$ engine load trials. We will conclude that the effect of changing the SPDI parameters at an engine load of $60 \%$, will not change the resulting pressure traces beyond the uncertainty of the experiment. The results will also show that the ID unit does create statistically significant higher cylinder pressures compared to the pressure resulting from ignition of the SPDI device.

\subsection{Varying SPDI Parameters}

The structure of the experiments was based on changing two parameters of the SPDI ignition device; spark duration (SL) and spark pulse power (SP and IP) ${ }^{1}$. For these experiments the SP and IP values were kept equivalent to each other. These results are compared and conclusions are drawn based on the pressure traces. All collected datasets include a minimum of 1000 combustion events, these were repeated on separate occasions to ensure repeatability. For all figures outlined in this section of the thesis, the resulting pressure versus volume plots are available in Appendix D.

\subsubsection{Changes in Plasma Duration}

Exposing the combustion chamber to the plasma for multiple durations was the first experiment. This meant starting with $\mathrm{SL}=40$ degrees then reducing this to 0 degrees, and determining if it had any effect on the combustion pressure traces. An example of the measured voltage and current traces for each SL setting $(0,20,40)$

\footnotetext{
${ }^{1}$ Please refer to Section 2.3 and Table 2.1, for the physical meanings of the abbreviations: $\mathrm{BC}$, SL, IL, IP, and SP.
} 
is illustrated in Figure 7.1, where in effect the entire spark durations are (starting at 20DBTDC) approximately 10 degrees, 30 degrees and 50 degrees.

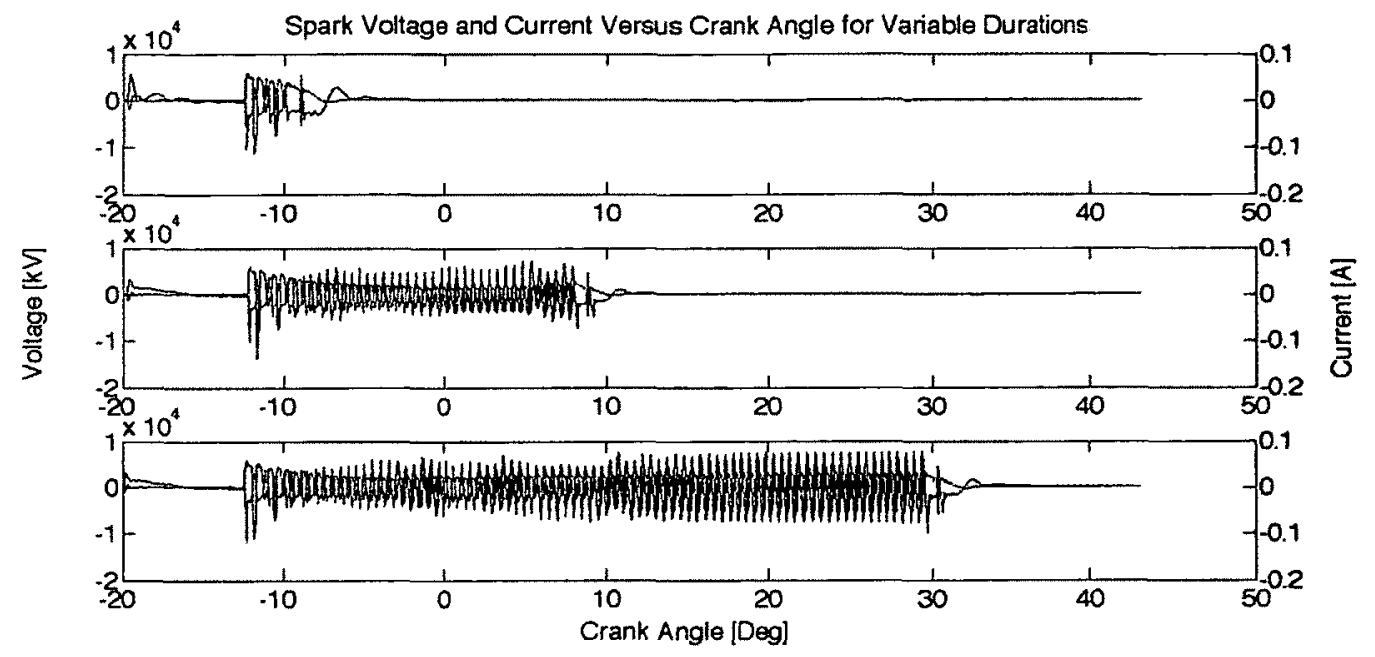

Figure 7.1: A plot of a typical spark voltage and current acquisition for three different SL settings $[0,20,40]$ starting at 20DBTDC. The current readings are inverse in polarity to coincide with the measurement method.

The first SPDI settings evaluated included; $\mathrm{BC}=50, \mathrm{SL}=\mathrm{IL}=[0,20,40]$, $\mathrm{SP}$ $=\mathrm{IP}=22$. This resulted in Figure 7.2. In this figure and many that follow, there will be three lines plotted for each dataset. The solid line corresponds to the mean of the data, the "upper limit" corresponds to the mean plus one standard deviation, and the "lower limit" corresponds to the mean subtract one standard deviation. 


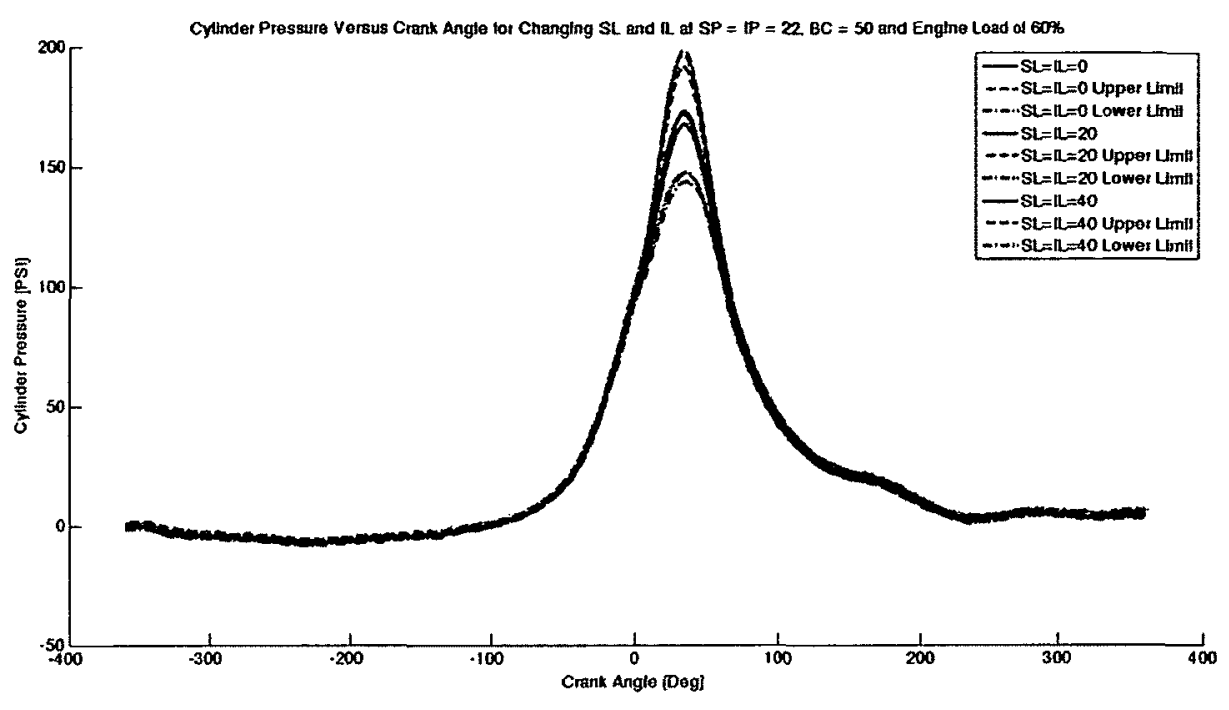

Figure 7.2: Cylinder pressure versus crank angle for three spark durations at SPDI settings of: $\mathrm{BC}=50, \mathrm{SL}=\mathrm{IL}=[0,20,40], \mathrm{SP}=\mathrm{IP}=22$, and engine load of $60 \%$. (see Table 2.1 on page 23 for a description of the SPDI settings)

The second set of SPDI parameters investigated included: $\mathrm{BC}=50, \mathrm{SL}=\mathrm{IL}=$ $[0,20,40], \mathrm{SP}=\mathrm{IP}=33$, this resulted in Figure 7.3 . 


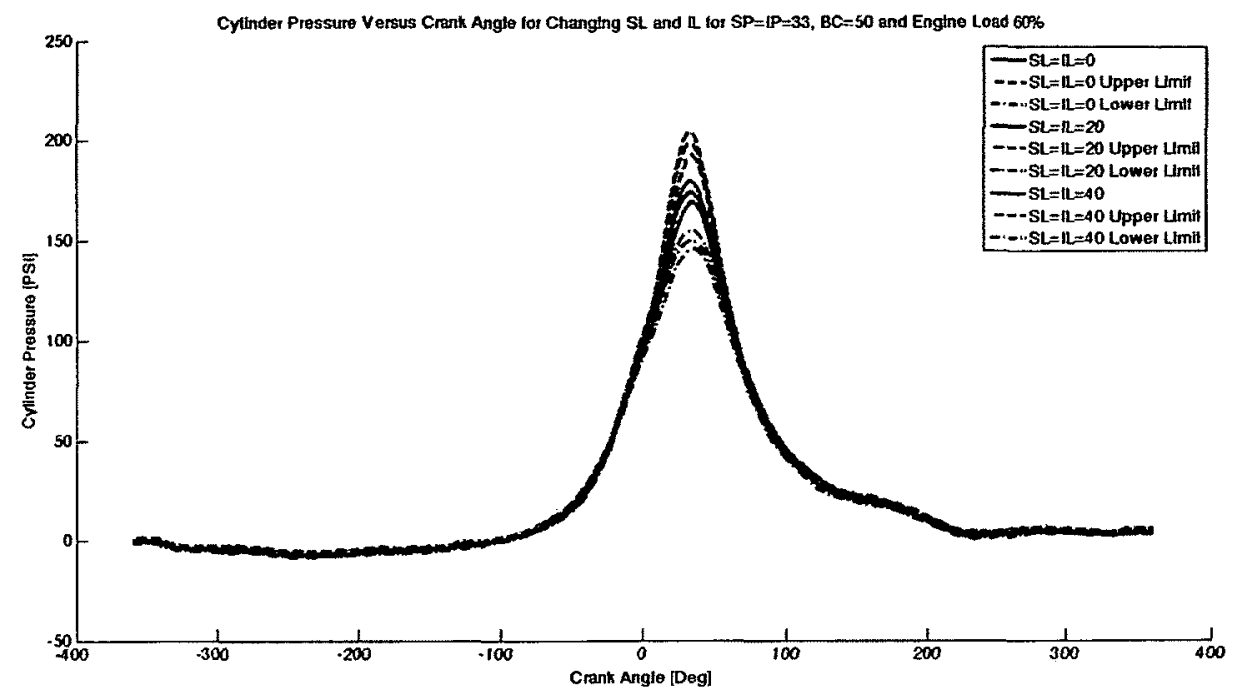

Figure 7.3: Cylinder pressure versus crank angle for three spark durations at SPDI settings of: $\mathrm{BC}=50, \mathrm{SL}=\mathrm{IL}=[0,20,40], \mathrm{SP}=\mathrm{IP}=33$, and engine load of $60 \%$. (see Table 2.1 on page 23 for a description of the SPDI settings)

The final set of SPDI parameters investigated included: $\mathrm{BC}=50, \mathrm{SL}=\mathrm{IL}=[0$, 20, 40], $\mathrm{SP}=\mathrm{IP}=44$, this resulted in Figure 7.4. 


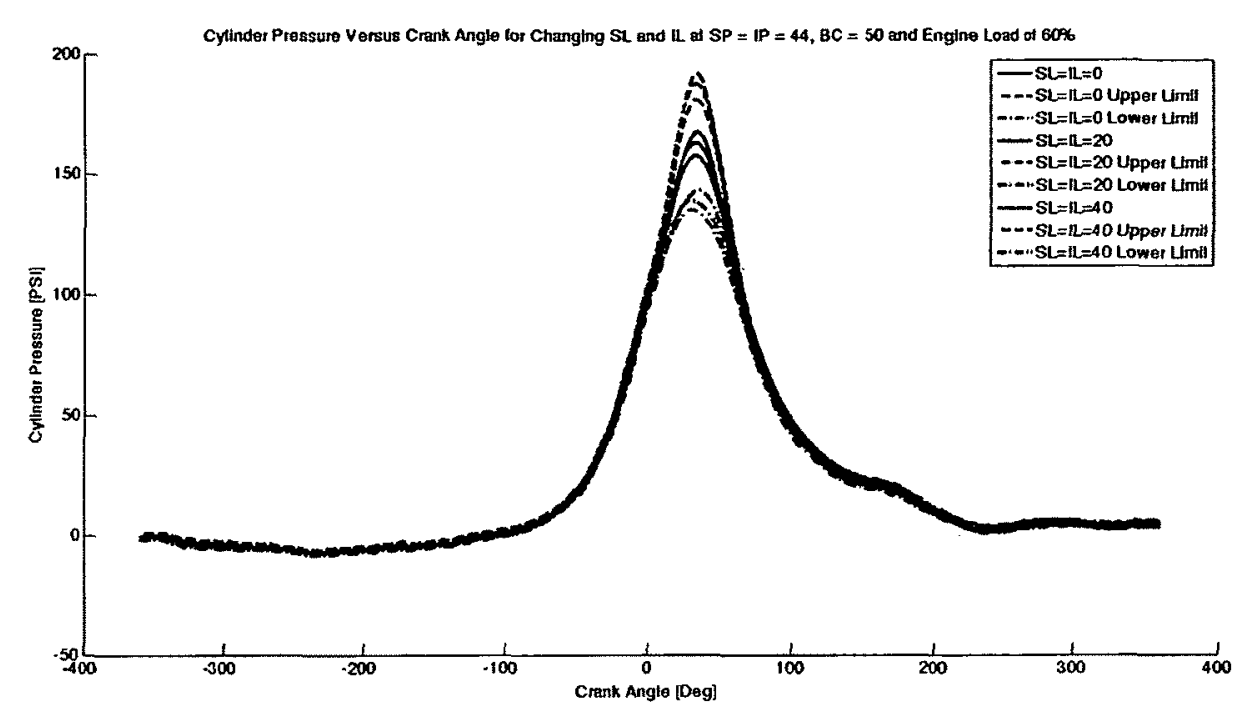

Figure 7.4: Cylinder pressure versus crank angle for three spark durations at SPDI settings of: $\mathrm{BC}=50, \mathrm{SL}=\mathrm{IL}=[0,20,40], \mathrm{SP}=\mathrm{IP}=44$, and engine load of $60 \%$. (see Table 2.1 on page 23 for a description of the SPDI settings)

According to the figures (7.2, 7.3 and 7.4), the pressure traces resulting from each spark duration at the SP and IP settings of 22,33 and 44 , show no differences beyond the experimental uncertainty (which is found to be $14.4 \%$ on average in Section 7.3 ).

In conclusion, for an ignition timing of 20 DBTDC any exposure from the SPDI spark to the combustion chamber for more than 15 degrees (i.e. 20DBTDC to 5DATDC) makes no statistical difference to the pressure traces beyond the experimental uncertainty. These results agree with those that were presented in the simulations from H.Do [21], where the effects of low-temperature plasma exposure to the flame propagation loses the dominance in production of radicals to assist in flame acceleration. It was noticed by H.Do that beyond the third or fourth pulse, the effects of the plasma diminish compared to the radical formation from the oxidation processes. Since the ignition system used here does not allow removal of the first 
4-5 pulses, the conclusions drawn are that exposure beyond the first 4-5 pulses have no effect on the combustion process outside the experimental uncertainty of $14.4 \%$ (average).

\subsubsection{Changes in Plasma Power}

This section will explore the effects of varying the SP and IP parameters on the measured cylinder pressure traces. By keeping the duration of the spark constant (i.e. $\mathrm{SP}=\mathrm{IP}=0$ ) at 10 degrees and only changing $\mathrm{SP}$ and IP parameters from 22 , to 33 and 44, the results of these experiments are presented in Figures 7.5.

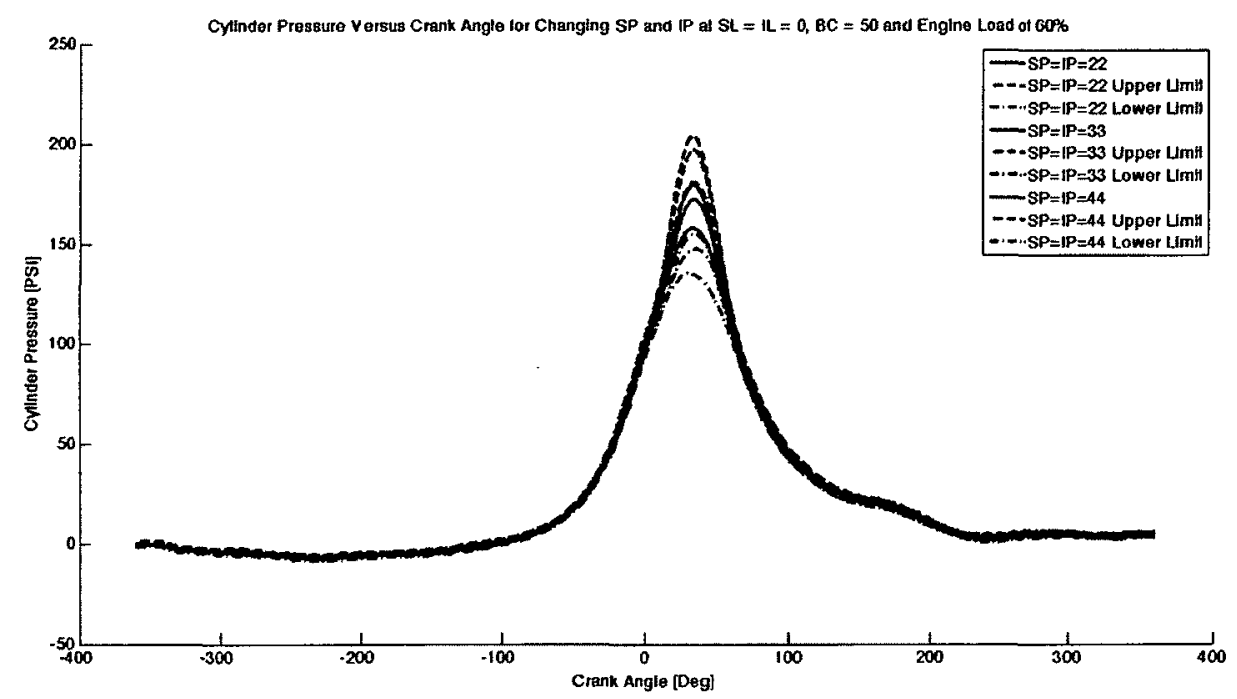

Figure 7.5: Cylinder pressure versus crank angle for three spark durations at SPDI settings of: $\mathrm{BC}=50, \mathrm{SL}=\mathrm{IL}=0, \mathrm{SP}=\mathrm{IP}=[22,33,44]$, and engine load of $60 \%$. (see Table 2.1 on page 23 for a description of the SPDI settings)

This test was also conducted for SPDI parameters of: $\mathrm{BC}=50, \mathrm{SL}=\mathrm{IL}=20, \mathrm{SP}$ $=I P=[22,33,44]$, this resulted in Figure 7.6. 


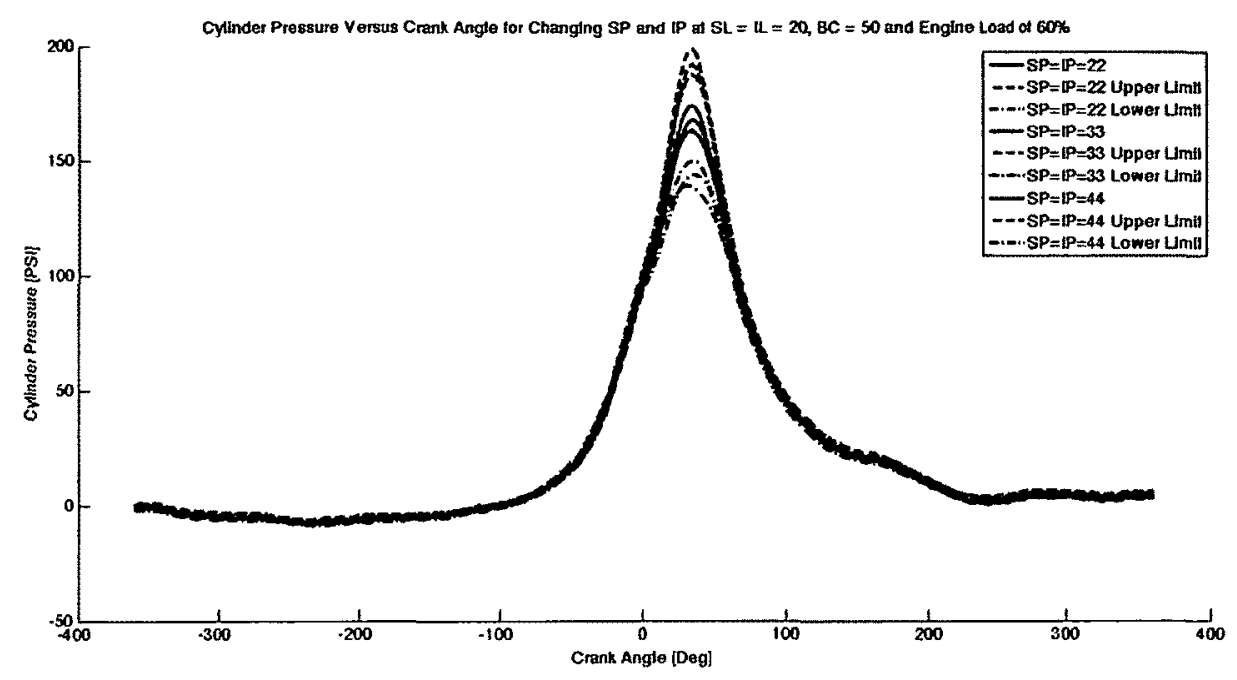

Figure 7.6: Cylinder pressure versus crank angle for three spark durations at SPDI settings of: $\mathrm{BC}=50, \mathrm{SL}=\mathrm{IL}=20, \mathrm{SP}=\mathrm{IP}=[22,33,44]$, and engine load of $60 \%$. (see Table 2.1 on page 23 for a description of the SPDI settings)

Finally, the test was conducted for SPDI parameters of: $\mathrm{BC}=50, \mathrm{SL}=\mathrm{IL}=40$, $\mathrm{SP}=\mathrm{IP}=[22,33,44]$, this resulted in Figure 7.7. 


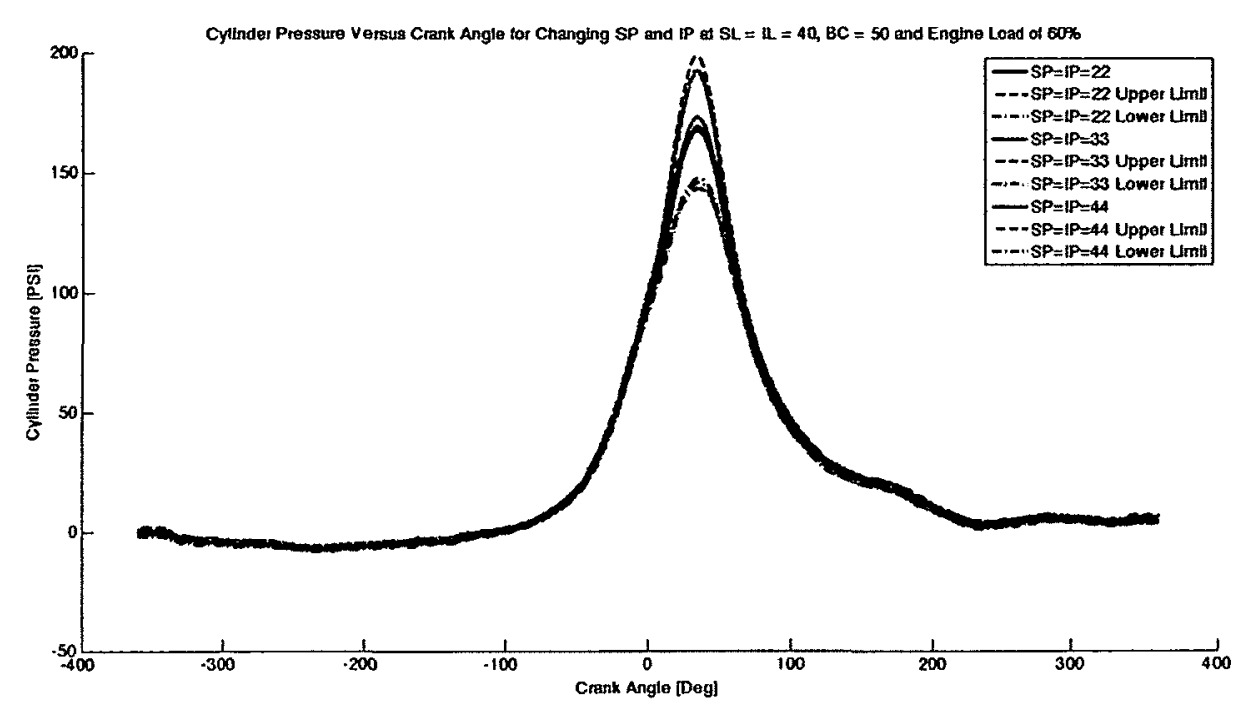

Figure 7.7: Cylinder pressure versus crank angle for three spark durations at SPDI settings of: $\mathrm{BC}=50, \mathrm{SL}=\mathrm{IL}=40, \mathrm{SP}=\mathrm{IP}=[22,33,44]$, and engine load of $60 \%$. (see Table 2.1 on page 23 for a description of the SPDI settings)

According to the results presented in Figure 7.5, the maximum mean pressure for $\mathrm{SP}=\mathrm{IP}=44$ is $16 \mathrm{PSI}$ below that of the other two trials presented in this figure. However the results rest as being statistically indifferent since they are not beyond the experimental uncertainty.

In conclusion, the results presented in this section of the thesis suggest that changing the SPDI parameters has less of an effect on the combustion process than what can be recorded by the experiment, this can be attributed to three possible reasons.

First, the experimental uncertainty is too large and the potential difference that the spark is creating on the combustion process is too small to be recorded by this apparatus. 
Second, it is possible that the plasma device does not create the postulated radical species to accelerate the combustion process as seen in literature. While it is hypothesized by the industrial partner and our research group, that the current pulse width of the unit (which is in the $\mu \mathrm{Sec}$ range) is able to create the expected electronically excited species, the results presented here only suggest that if there exists electronically excited species, their effects on the combustion process are less than the experimental uncertainty ( $14.4 \%$ on average). This however is only speculation based on the combustion traces, research into determining the plasma characteristics is reserved for another project.

The third possible reason for not seeing changes in the pressure traces could be due to the use of an arc discharge before application of the plasma. While other researchers $([21])$ have shown a depreciating effect of the plasma on the combustion process after the first few pulse, further delaying the plasma through the use of an arc discharge could inhibit the postulated radical formations into the already expanding flame kernel. This conclusion would result in a lesser effect from plasma application to the combustion process since excited species would not be able to reach the unburnt fuel and air mixture. Most researchers (e.g [10], [11] and [12]) in the area of plasma assisted combustion do not use an arc discharge coupled to their plasma devices, but instead initiate combustion solely from the plasma. In contrast to the SPDI device, this would allow for the creation of radical species prior to ignition in the environment of unburnt fuel and air mixture. 


\subsection{Spatial Plasma Discharge Ignition Versus Conventional Inductive Discharge Ignition}

Previous results have shown that changing SPDI parameters has no statistically significant effect on the pressure traces in the combustion chamber. Therefore the comparison of SPDI versus ID is made simpler by only using two SPDI parameter settings; SPDI Profile 1 and SPDI Profile 2.

SPDI Profile 1: $\mathrm{BC}=100, \mathrm{SL}=\mathrm{IL}=0, \mathrm{SP}=\mathrm{IP}=22$,

SPDI Profile 2: $\mathrm{BC}=50, \mathrm{SL}=\mathrm{IL}=0, \mathrm{SP}=\mathrm{IP}=22$,

This portion of the thesis will therefore compare the resulting pressure traces of these SPDI profiles to that of the conventional ID device. Upon conducting these tests, the resulting initial currents (at ignition system trigger) were measured for the SPDI profiles and the ID spark.

Table 7.1: Measured spark currents for two SPDI system settings and the conventional ID system

\begin{tabular}{|l|l|}
\hline Spark Type & Resulting Initial Current \\
\hline \hline SPDI Profile 1 & $15 \mathrm{~mA}$ (measured) BC $=100$ \\
SPDI Profile 2 & $25 \mathrm{~mA}$ (measured) BC $=50$ \\
ID & $50 \mathrm{~mA}$ (measured) \\
\hline
\end{tabular}


Figure 7.8 depicts the results of these experiments at an engine load of 1180 Watts.

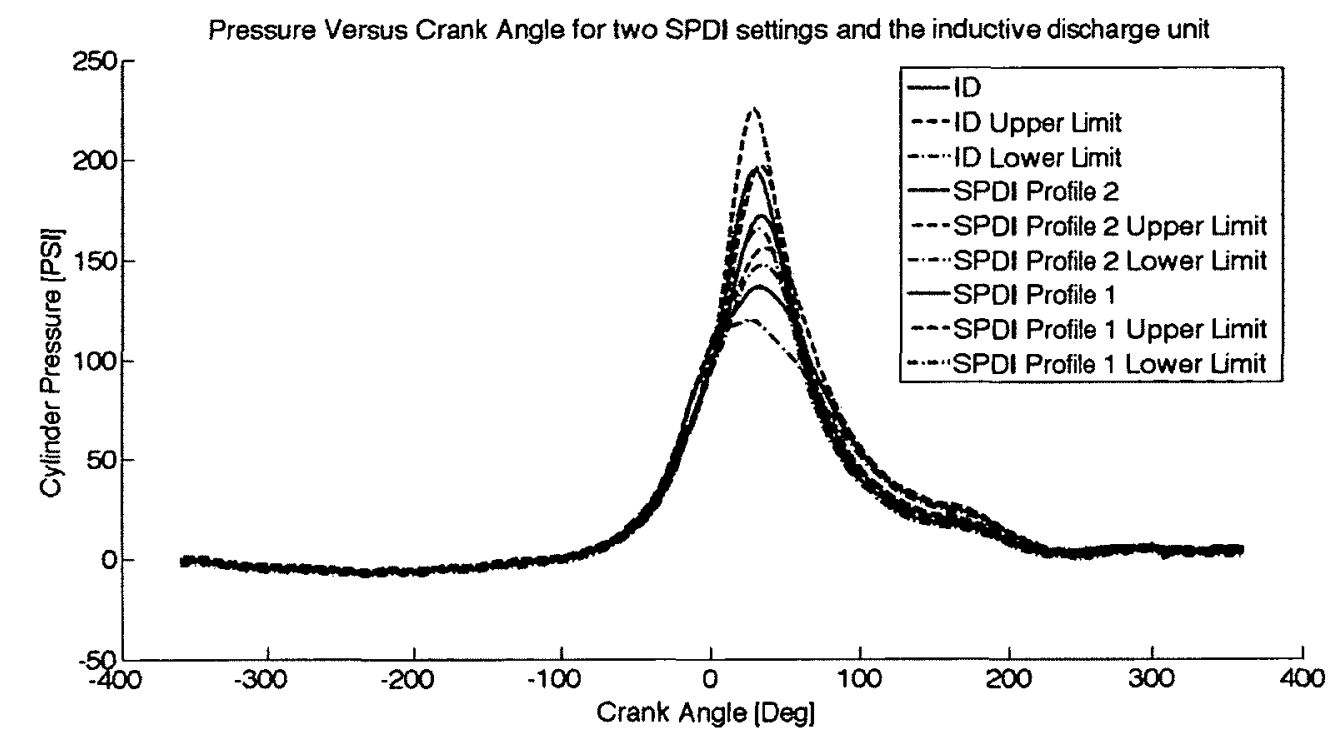

Figure 7.8: Cylinder pressure versus crank angle for three trials at an engine load of $60 \%$. (see Table 2.1 on page 23 for a description of the SPDI settings)

It is clear in these results that an increase in measured initial current changes the pressure traces found in the combustion chamber. The maximum pressure achieved by each trial is outlined in Table 7.2

Table 7.2: Maximum cylinder pressures achieved by ignition from two SPDI spark profiles and the conventional ID system

\begin{tabular}{|l|l|l|}
\hline Spark Type & Max.Pressure[PSI] & $\sigma[\mathrm{PSI}]$ \\
\hline \hline SPDI Profile 1 & 136.4 & 18.3 \\
SPDI Profile 2 & 172.3 & 25 \\
ID & 195 & 30.4 \\
\hline
\end{tabular}


Since the results are beyond the experimental uncertainty, the MFB analysis outlined in Section 3.3 can be used. Figure 7.9, depicts the resulting MFB curves for the trials depicted in Figure 7.8.

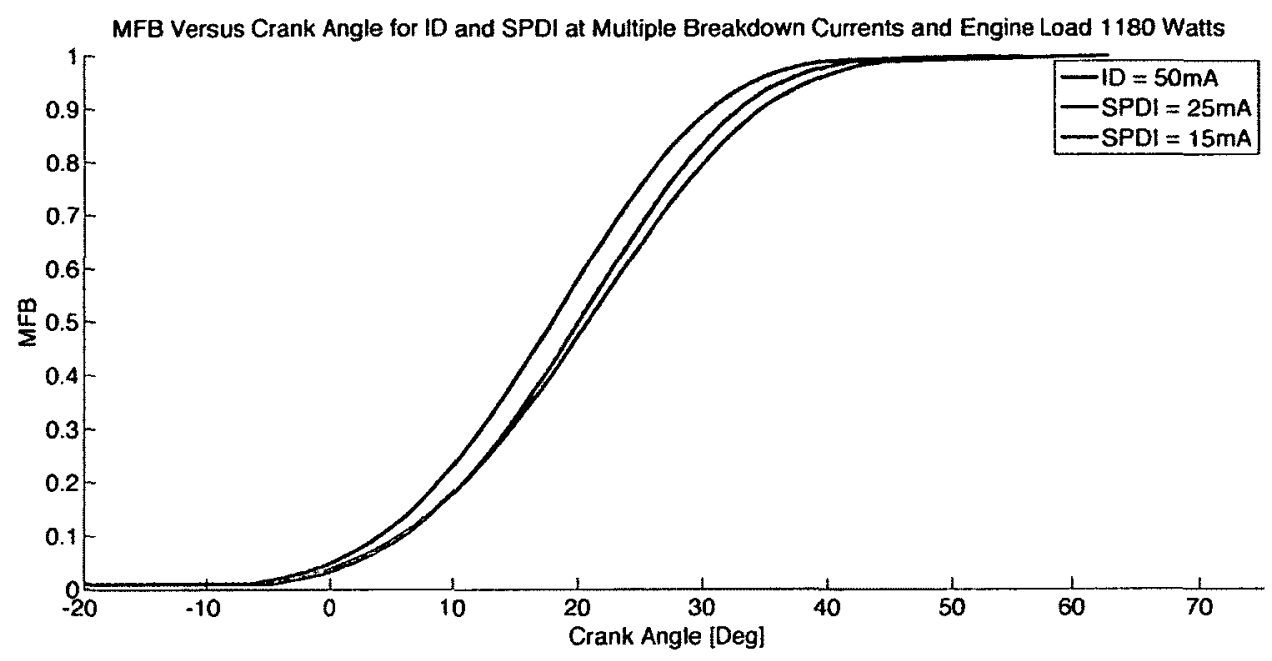

Figure 7.9: A plot of a typical spark voltage and current acquisition with the parameters identified.

However, as per the discussion in section 6.4 there may exist underlying nonGaussian distribution shapes and thus a mean curve may not best describe the data. Therefore a histogram of the crank angle locations at $\mathrm{MFB}=0.5$ (Figure 7.10), the rate of $\mathrm{MFB}$ change at $\mathrm{MFB}=0.5$ (Figure 7.11), and the crank angle corresponding to the EOC location (Figure 7.12) are included to depict the true differences between each MFB curve. 


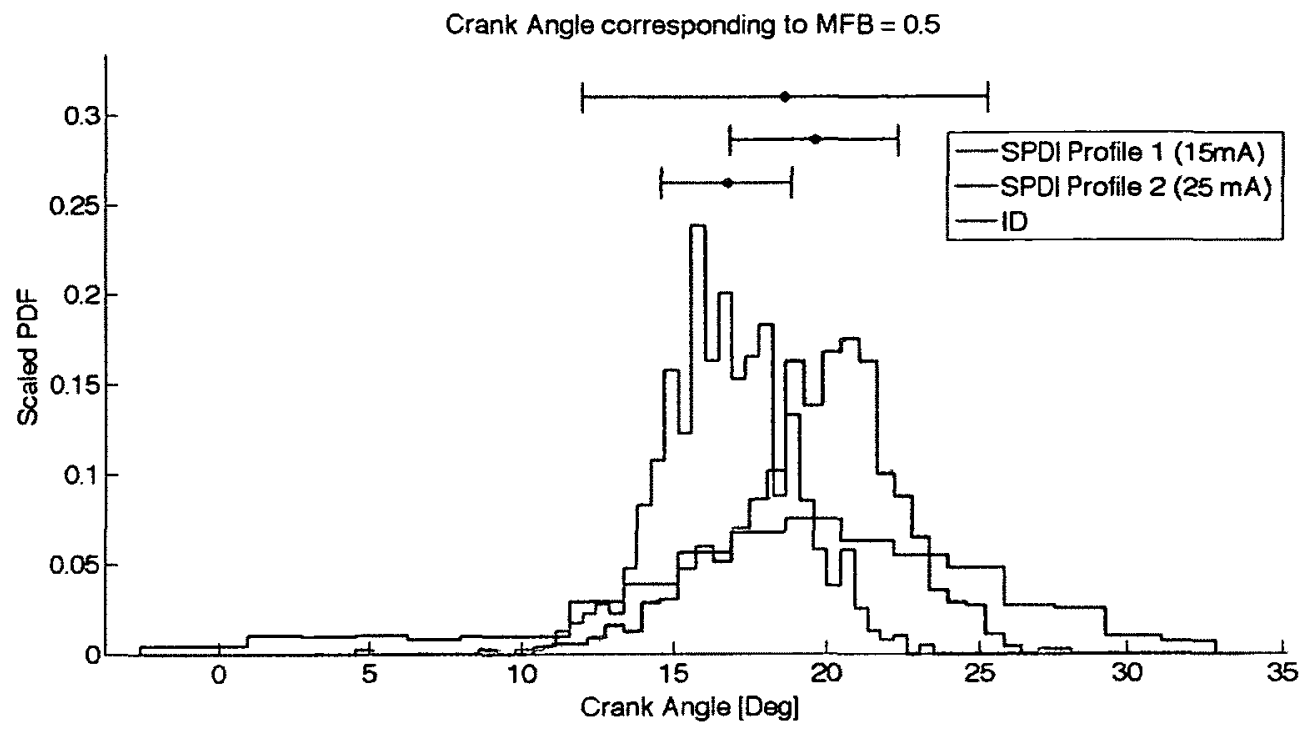

Figure 7.10: A histogram of crank angle values corresponding to $\mathrm{MFB}=0.5$.

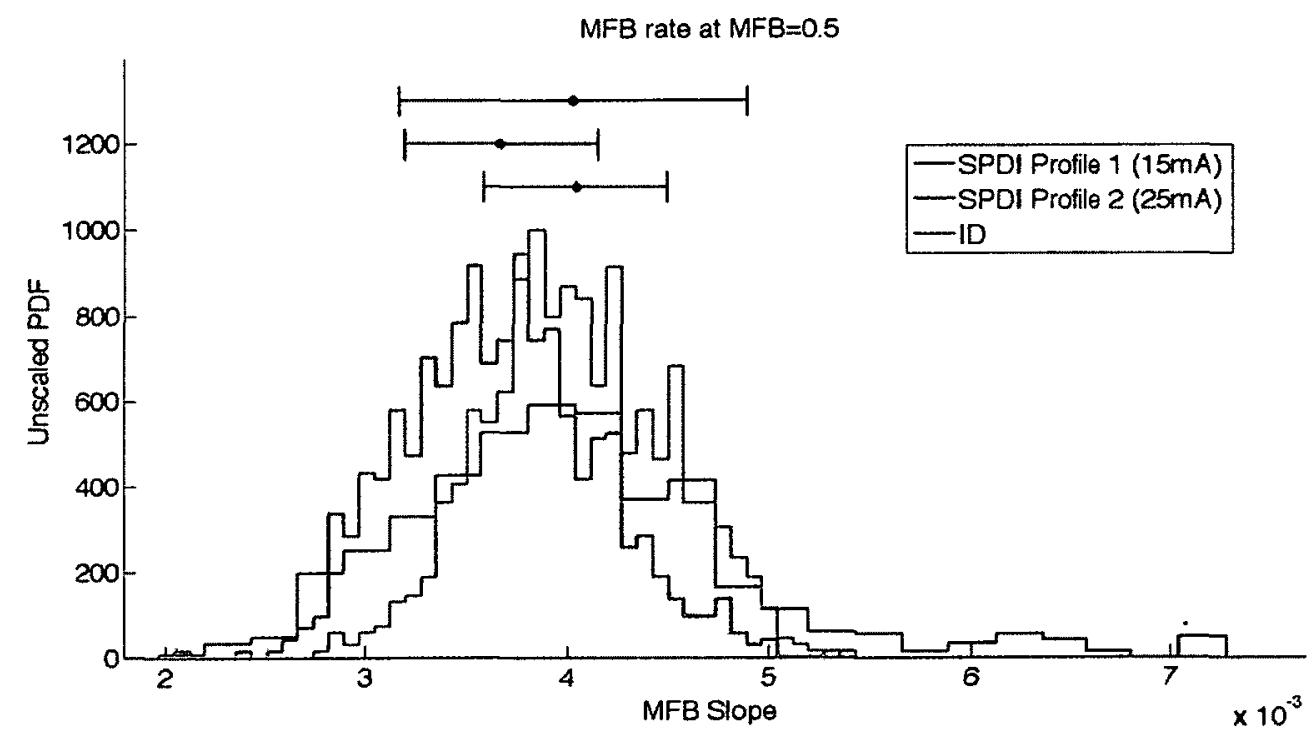

Figure 7.11: A histogram of resulting MFB slope $\left[\frac{\mathrm{MFB}}{\mathrm{Deg}}\right]$ at $\mathrm{MFB}=0.5$. 


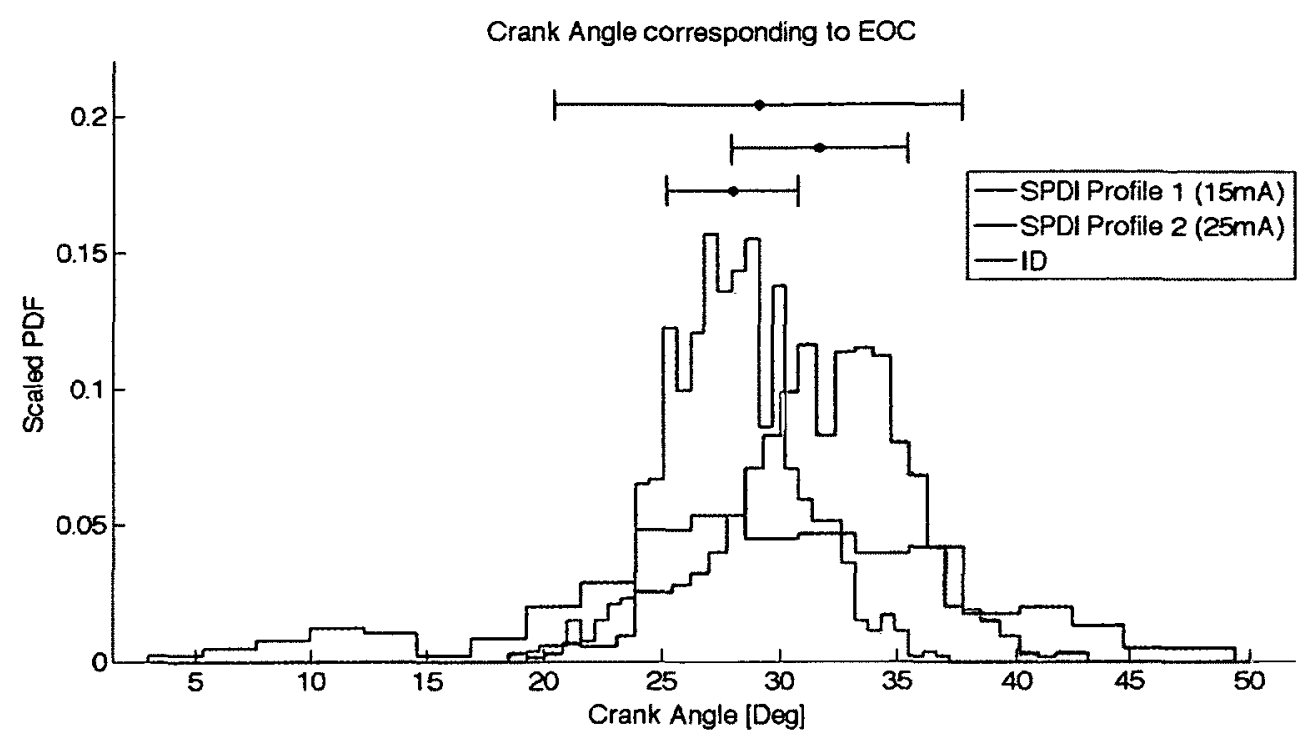

Figure 7.12: A histogram of crank angles corresponding to EOC locations.

Firstly, the histogram distribution shapes depicted in Figures 7.10 to 7.12 , show that no underlying bimodal or skewed distributions exist and therefore the method of taking a mean value to approximate the dataset is validated. The results however show that large cyclic variability is present when using the SPDI profile 1 with measured breakdown current of $15 \mathrm{~mA}$, this is speculated to be due to inadequate ignition of the fuel and air mixture thus resulting in lower maximum pressures. As well, the MFB histograms (Figures 7.10 to 7.12) depict that the standard deviations overlap for all three shape characteristics of the MFB curves and thus render the MFB results inconclusive. These MFB results however do not affect the maximum pressure conclusions discussed earlier. 


\subsection{Two Tail T-Test}

In experimental investigations it is recommended that statistical analyses be conducted on the results to ensure the quality of the conclusions. This section will outline the two tailed t-test analysis method used in this investigation as well as the applicable conclusions that are drawn from it.

\subsubsection{Methods}

The two tailed t-test is designed to calculate the probability that two population means are equivalent. It is based upon using,

$$
t=\frac{\left(\bar{x}_{1}-\bar{x}_{2}\right)-d_{0}}{s_{p} \sqrt{\frac{1}{n_{1}}+\frac{1}{n_{2}}}}
$$

where

$$
s_{p}^{2}=\frac{s_{1}^{2}\left(n_{1}-1\right)+s_{2}^{2}\left(n_{2}-1\right)}{n_{1}+n_{2}-2}
$$

$\bar{x}_{1}$ and $\bar{x}_{2}$ are the sample means, $n_{1}$ and $n_{2}$ are the sample population sizes and $d_{0}$ is the difference between the population means (i.e. $d_{0}=\mu_{1}-\mu_{2}$ ). The assumptions used in this test require that the populations be of the normal distribution form and that the population variances be equivalent.

From here the two tailed hypothesis is not rejected when

$$
-t_{\alpha / 2, n_{1}+n_{2}-2}<t<t_{\alpha / 2, n_{1}+n_{2}-2}
$$

where $\alpha=0.05$. It should be noted that the parameter, $d_{0}$ in Equation 31, plays a dominant role in the result of $t$ and if equal to zero, represents two statistical 
distributions that are the same $\left(d_{0}=0=\mu_{1}-\mu_{2}\right)$. Therefore, as $d_{0}$ increases, the result is an acceptance of a larger difference between the two population means which are still said to be equivalent.

\subsubsection{Choosing $d_{0}$}

Based upon the pressure trace results discussed in Section 7.1and 7.2, it can be seen that the pressure trace standard deviations follow the same trend, increasing between TDC and 35DATDC then decreasing between 35DATDC to 60DATDC. Plotting the standard deviations for all trials investigated versus their crank angle and taking the mean (Figure 7.13), the result is that at a crank angle of 33.2DATDC the maximum average standard deviation occurs, 24.8 PSI.

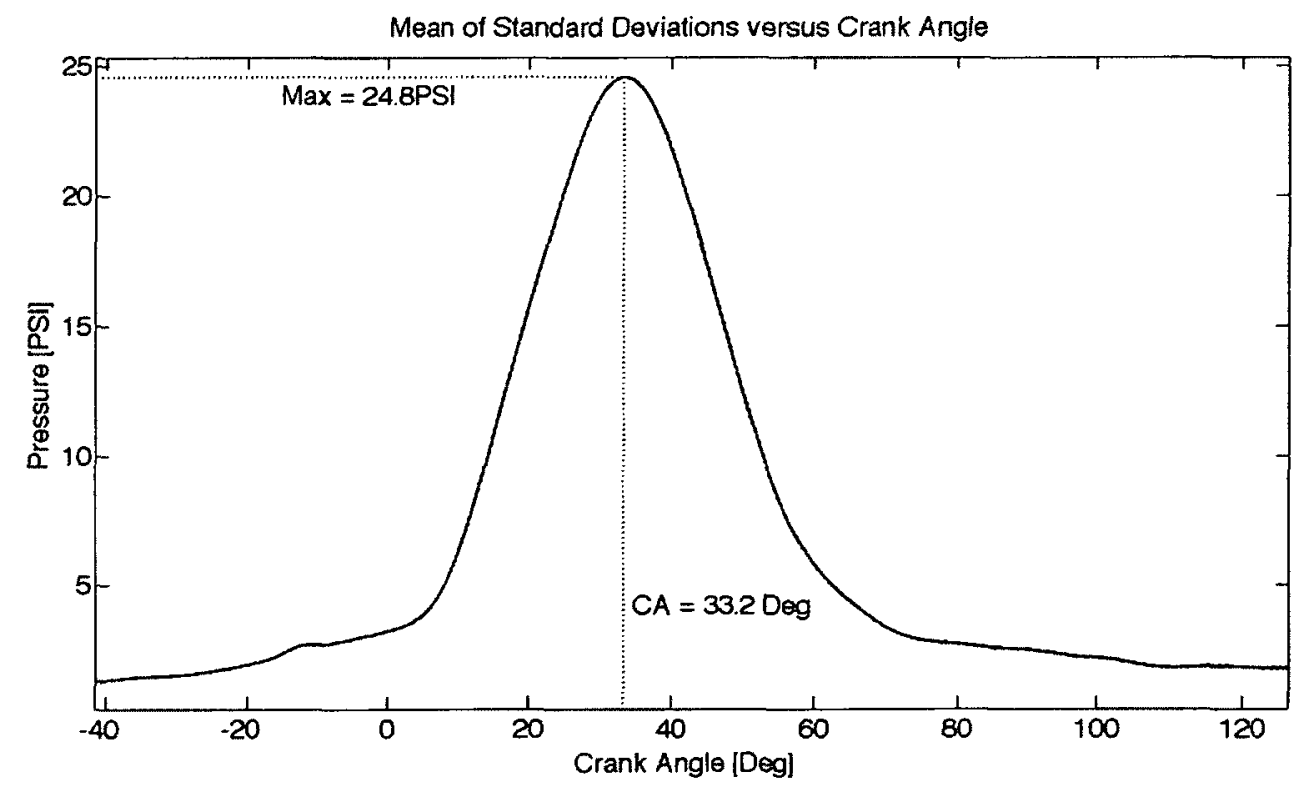

Figure 7.13: Plot of average standard deviation from their respective cylinder pressure means versus crank angle. 
Using this same concept however plotting the result as a function of percentage cylinder pressure, the result is that the average maximum standard deviation is $14.4 \%$ (Figure 7.14). Therefore it seems sensible to say that the maximum difference between two population means should be no more than 24.8PSI if they're going to be identified as statistically equivalent in this investigation. From this analysis, the resulting $d_{0}$ for the experiment will be $d_{0}=24.8$ PSI as any less would not be identifiable by the experiment apparatus.

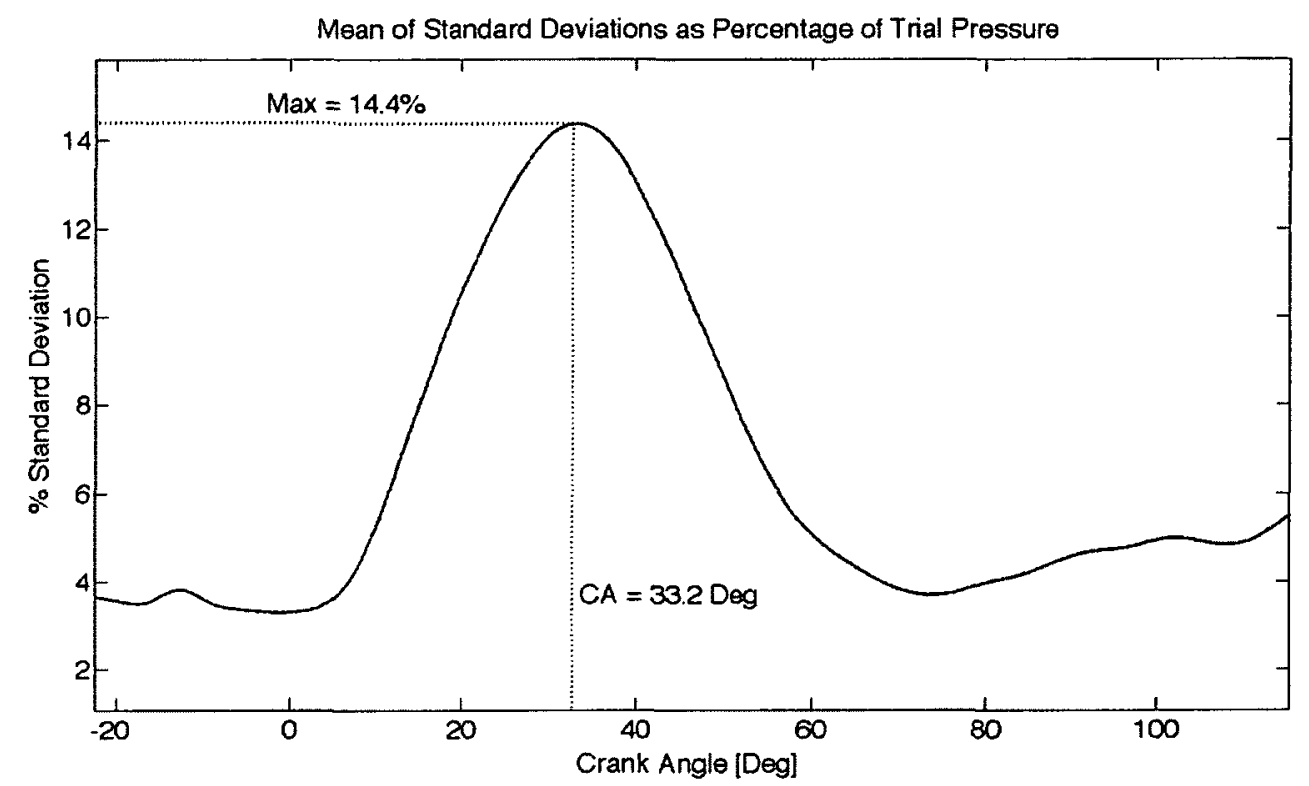

Figure 7.14: Plot of average percent standard deviation from their respective cylinder pressure means versus crank angle.

\subsubsection{T-Test Results}

For every angular location between 20DBTDC and 60DATDC, there is a $d_{0}$ value which results in the t-test hypothesis being accepted, however large or small. Therefore when a t-test is conducted on each of the trials at all crank angle locations, 
the minimum $d_{0}$ is calculated to discern the point at which the hypothesis results in being accepted. This can be seen by a $d_{0}$ versus crank angle plot (Figure 7.15) for each trial, where every curve corresponds to a comparison between two pressure trace results from the Figures in Sections 7.1and 7.2. In Figure 7.15, for the curves below the threshold of 24.8 PSI, the result is that for those trials the comparisons resulted in the hypothesis being accepted and thus the means are said to be equivalent to within the accuracy of the experiment. For the trials who's minimum $d_{0}$ are larger than 24.8 PSI, the result is that for those crank angles the mean of the distributions are statistically different. The final conclusions that can be drawn from this analysis are that the ID trials seen in Figure 7.8 are statistically different than the SPDI trials of Figure 7.8 (tabulated in Table 7.3) and that the remainder of the experiments are shown to be statistically the same to within the experimental uncertainty (14.4\%). 
Table 7.3: Results of T-test statistic comparing ID to both SPDI Profile 1 and SPDI Profile 2 trials. For each interval of rejection, the curves are said to be statistically NOT EQUIVALENT

\begin{tabular}{|c|c|c|}
\hline Trial & $\begin{array}{c}\text { Compared } \\
\text { Trial }\end{array}$ & $\begin{array}{c}\text { Crank Angle } \\
\text { of Hypothesis } \\
\text { Rejection }\end{array}$ \\
\hline ID & SPDI Profile 1 & $\begin{array}{l}\text { 13.9 DATDC to } \\
\text { 44 DATDC }\end{array}$ \\
\hline ID & SPDI Profile 2 & $\begin{array}{l}\text { 14.4 DATDC to } \\
\text { 39.6 DATDC }\end{array}$ \\
\hline
\end{tabular}

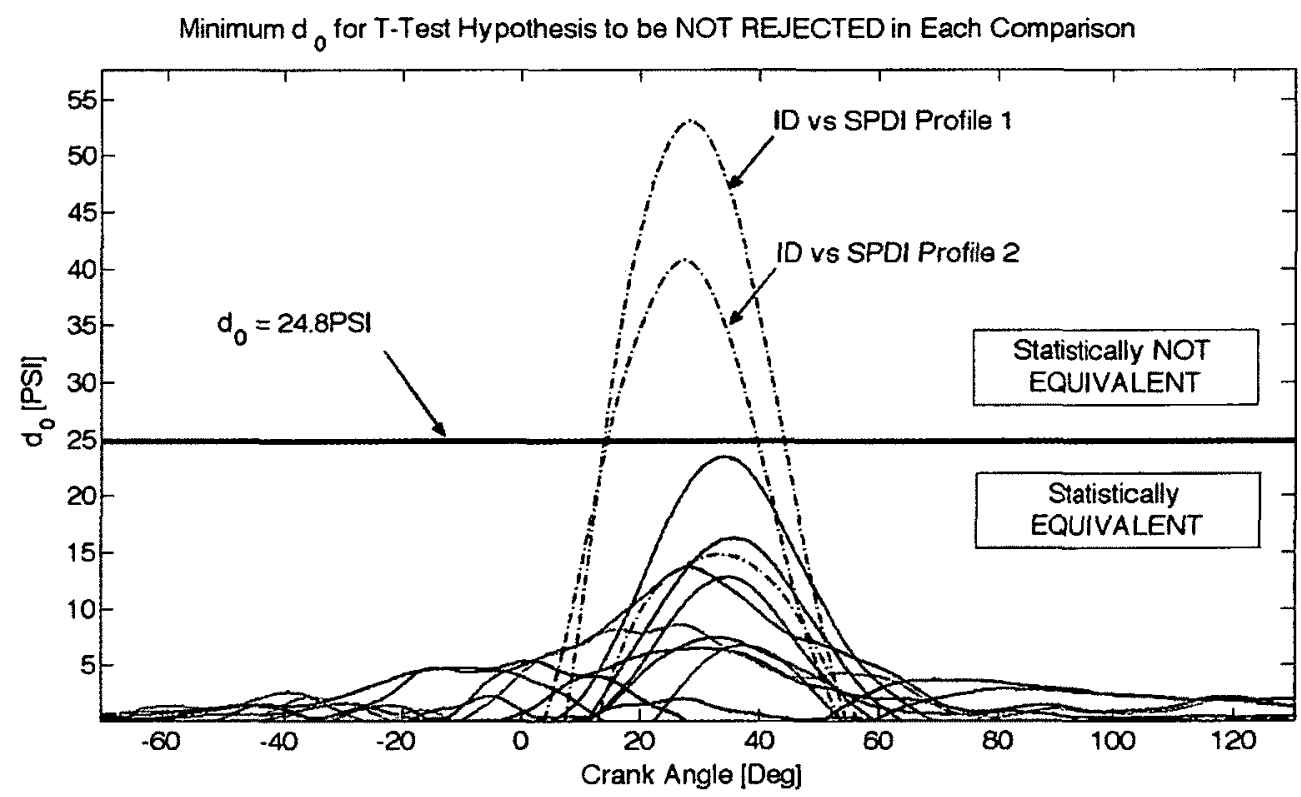

Figure 7.15: Minimum $d_{0}$ for comparisons between each trial such that the hypothesis is not rejected. If this $d_{0}$ is below the 24.8 PSI threshold, the comparison results in statistically equivalent curves. If the $d_{0}$ is above the 24.8 PSI threshold, the comparison results in statistically NOT equivalent curves 


\subsection{The SPDI Spark Profile}

The pressure traces in this chapter are a result of ignition from the SPDI device at various user interface settings. The voltage and current curves gathered during these experiments have shown that there exists two locations where breakdown occurs between the electrodes. The first location is upon the start of the "Dwell Duration" identified in Figure 2.6 where the measured breakdown currents of $15 \mathrm{~mA}$ and $25 \mathrm{~mA}$ were gathered for the SPDI trials of Section 7.2. The second is upon the start of the "Ignition Duration" in Figure 2.6. It is noteworthy that the BC setting in the SPDI user interface changes the breakdown current measured at the beginning of the "Ignition Duration" portion of the spark. Furthermore, the result of measuring the input energy for the first pulse in the "Dwell Duration" component of the spark profile, is a maximum of $2 \mathrm{~mJ}$ in energy. This however is compared to the result at the beginning of "Ignition Duration", which is $9 \mathrm{~mJ}$ of energy. Since flame kernel formation is highly dependent on this energy quantity, this can therefore result in a delayed ignition of the fuel-air mixture by a value of $500 \mu \mathrm{sec}$ (from Figure 2.6). Further investigation into the flame kernel formation characteristics resulting from the SPDI device ignition through use of an optically accessible combustion chamber will be a subject of future research.

\subsection{Engine Experiment Conclusions}

In conclusion, the SPDI device can be statistically proven to create lower cylinder pressures (using SPDI Profile 1) at the same load as a conventional ID system. Although the burn characteristics according to the MFB analysis show that for the 
SPDI ignition and conventional ID ignition there exists no statistically significant results beyond the experimental uncertainty of $14.4 \%$. However, the histograms presented here suggest that there could exist an underlying trend in which the inductive discharge unit creates lower ignition delay times (seen by the lower crank angle mean of the distributions in Figure 7.10) when compared to the SPDI device. The hypothesis that the pulses during flame kernel formation affect the rate of combustion after ignition by altering the initial chemical reaction pathways, according to Figure 7.11, shows that no statistical significance exists, nor does any underlying trend appear. However, as discussed in Section 7.4 these results can be a consequence of the SPDI spark profile used during this investigation. 


\section{Chapter 8}

\section{Conclusion}

\subsection{Summary}

The work presented here involved two major experiments to prove firstly, that the ignition system (SPDI) used in this research formed a plasma between the electrodes of a spark plug, and secondly, that the addition of plasma to an arc discharge could affect the combustion process inside a combustion chamber of a single cylinder engine. It has been postulated by the industrial partner that the use of the SPDI device can enhance engine performance by $4 \%$ to $9 \%$ when compared to a conventional inductive discharge unit.

\subsubsection{Imaging the Ignition Systems}

This research made use of a high speed nanosense camera that captured images of the spark progression between the electrodes. Two experiments were conducted, one at ambient pressure and one in pressurized conditions between OPSI and 150PSI. The results for the ambient condition experiment show that the SPDI device is capable of 
creating and sustaining a luminous plasma conduit between the electrodes of a spark plug. In the investigation at higher pressures it was concluded that the SPDI device could sustain the luminous plasma conduit as well as a non-luminous discharge state. These two discharge states, luminous and non-luminous are speculated to be a glow discharge (below 110PSI) and a streamer discharge (above 110PSI), respectively. It was also concluded that the type of plasma emitted during the use of the SPDI device to ignite a fuel and air mixture was not constant throughout the spark, and that the resulting voltage and current traces were highly dependent on localized pressure waves between the electrodes in the combustion chamber.

\subsubsection{Single Cylinder Engine Pressure Trace Analysis}

The work presented here made use of a single cylinder engine in which two ignition systems (SPDI unit and a conventional ID unit) were mounted and used to ignite the air and fuel mixture inside the combustion chamber. This experiment involved the development of a hardware ignition timing program that would provide constant and accurate timing for the engine, as well it had the purpose of monitoring the engines performance parameters and logging the datasets.

The first experiment used only the SPDI ignition system. The engine cylinder pressure was monitored when variations of the SPDI device parameters were changed including spark duration and spark power. The standard deviation of the experimental data is $14.4 \%$ which is greater than the $4 \%$ to $9 \%$ impact of changing the SPDI spark attributes postulated by the industrial partner. The impact on the combustion process by changing the SPDI attributes can therefore not be determined with the data collected in this experiment. Thus the hypothesis made by the industrial 
partner cannot be confirmed or disproved due to data uncertainty.

The second experiment compared the resulting cylinder pressure traces of the SPDI ignition system and the conventional ID system. The results showed that the SPDI system was able to create statistically significant lower cylinder pressures while maintaining the engine load requirement. This however was found to be at a cost of cyclic variability and thus engine instability. When analyzing the data through mass fraction burn (MFB) techniques it was concluded that the differences between the combustion characteristics were not statistically significant beyond the $14.4 \%$ experimental uncertainty of the apparatus.

\subsubsection{Future Work}

\section{Imaging the Ignition Systems}

While it was an advancement to determine that the ignition system used here was able to create a plasma conduit, it is recommended that future students classify the two types of plasma that are produced by the ignition system. It is postulated by the manufacturer that it is able to create radical species through electronic excitation of the molecules, however an investigation into the validity of this is recommended.

\section{Engine Cylinder Pressure Analysis}

The mass fraction burn (MFB) analysis outlined in this thesis is relatively rudimentary and development for better approximation of the polytropic index during the expansion stroke of the engine is suggested to be investigated. As well, a comparative method of determining end-of-combustion locations is also recommended to ensure 
more accurate results of the MFB analysis.

For future work into monitoring the engine parameters, it is recommended that the students make use of a multi-cylinder engine to reduce the cyclic variability of the engine. As well it is suggested that a water-brake system be employed to allow better control of the load on the engine. The software developed in this paper will be made available through my supervisor (Dr. Ronald Miller) to students who are interested in using the engine timing and engine analysis programs I have developed. 


\section{Appendix A}

\section{Instrumentation Information}




\section{A.1 Optrand AutoPSI-S fibre Optic Pressure Sen-} sor

Table A.1: Calibration specifications and details pertaining to the Optrand AutoPSI-S fibre optic pressure transducer.

\begin{tabular}{|l|l|}
\hline Parameter & Specification \\
\hline \hline Model No. & D22294-Q \\
S/N & $7728 \mathrm{~B}$ \\
Pressure Range & $0-3000 \mathrm{PSI}$ \\
Install Torque & $15-17 \mathrm{in}-\mathrm{lbs}$ \\
Input Voltage & $9-18 \mathrm{~V}$ \\
Output Voltage & $0.5-4.5 \mathrm{VDC}$ \\
Sensitivity & $3.89 \mathrm{mV} / \mathrm{PSI}$ at $200^{\circ} \mathrm{C}$ \\
\hline
\end{tabular}




\section{A.2 MKII Nanosense High Speed Camera}

\begin{tabular}{|c|c|c|}
\hline \multicolumn{3}{|c|}{ Speciffcations for standard version } \\
\hline 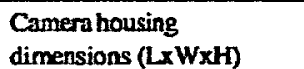 & \multicolumn{2}{|c|}{$\begin{array}{l}165 \times 95 \times 95 \mathrm{~mm} \\
\text { Excl. cable connectors }\end{array}$} \\
\hline Weighe & \multicolumn{2}{|l|}{$\sim 1900 \mathrm{gr}$} \\
\hline Lens mount & \multicolumn{2}{|l|}{ C-mount } \\
\hline Sensor type & \multicolumn{2}{|l|}{ CMOS } \\
\hline Active pixels & \multicolumn{2}{|c|}{$512 \times 512$ pixels } \\
\hline Camera bit resolution & \multicolumn{2}{|c|}{ 10-bit (typical B-bit readiour } \\
\hline Pixel size and pitch & \multicolumn{2}{|l|}{$16 \times 16 \mu \mathrm{m}$} \\
\hline Pixel fill & \multicolumn{2}{|l|}{$62 \%$} \\
\hline $\begin{array}{l}\text { Quantum efficiency } \\
\text { Dynamic range }\end{array}$ & \multicolumn{2}{|c|}{$\begin{array}{l}41 \% \text { @ } 532 \mathrm{~nm} \\
59 \mathrm{~dB} \\
\end{array}$} \\
\hline Sync. $\ln /$ an & \multicolumn{2}{|c|}{ CMOS via BNC (3.3V) } \\
\hline Inter frame time (PIV) & \multicolumn{2}{|l|}{$100 \mathrm{~ns}$} \\
\hline $\begin{array}{l}\text { Integration time } \\
\text { (exposure) }\end{array}$ & \multicolumn{2}{|c|}{$1000 \mathrm{~ns}-100 \mathrm{~ms}$} \\
\hline \multirow[t]{6}{*}{ Resolution and Frame rate } & Resolution & $\begin{array}{l}\text { Frame rate } \\
\text { [Hz] }\end{array}$ \\
\hline & $512 \times 512$ & 5130 \\
\hline & $512 \times 256$ & 10100 \\
\hline & $512 \times 128$ & 20100 \\
\hline & $512 \times 64$ & 38500 \\
\hline & $512 \times 16$ & 132000 \\
\hline Camera interface & \multicolumn{2}{|l|}{ USB2 } \\
\hline $\begin{array}{l}\text { Fast storage RAM } \\
\text { addressable for multiple } \\
\text { acquisitions }\end{array}$ & \multicolumn{2}{|c|}{$\begin{array}{l}8191 \text { full resolution images } \\
\text { Or } \\
16382 \text { full resolution inages }\end{array}$} \\
\hline \multicolumn{3}{|c|}{ Plus mode enhanced specifications } \\
\hline \multirow[t]{5}{*}{ Resolution and Frame rate } & Resolution & $\begin{array}{l}\text { Frame rate } \\
\text { (Hz) }\end{array}$ \\
\hline & $\begin{array}{l}512 \times 512 \\
512 \times 256\end{array}$ & $\begin{array}{l}10260 \\
20200\end{array}$ \\
\hline & $512 \times 128$ & 40200 \\
\hline & $512 \times 64$ & 77000 \\
\hline & $512 \times 16$ & 264000 \\
\hline Camera interface & \multicolumn{2}{|c|}{$\begin{array}{l}\text { USB2 } \\
\text { and } \\
\text { GEthernet for fast inge transfer }\end{array}$} \\
\hline $\begin{array}{l}\text { Fast storage RAM } \\
\text { addressable for multiple } \\
\text { acquisitions }\end{array}$ & \multicolumn{2}{|c|}{$\begin{array}{l}16000 \text { full resolution images } \\
\text { Or } \\
32000 \text { full resolution images }\end{array}$} \\
\hline
\end{tabular}

Figure A.1: Sample Specifications for the Dantec Nanosense MKII High Speed CCD camera. 


\section{A.3 SPDI User Interface}

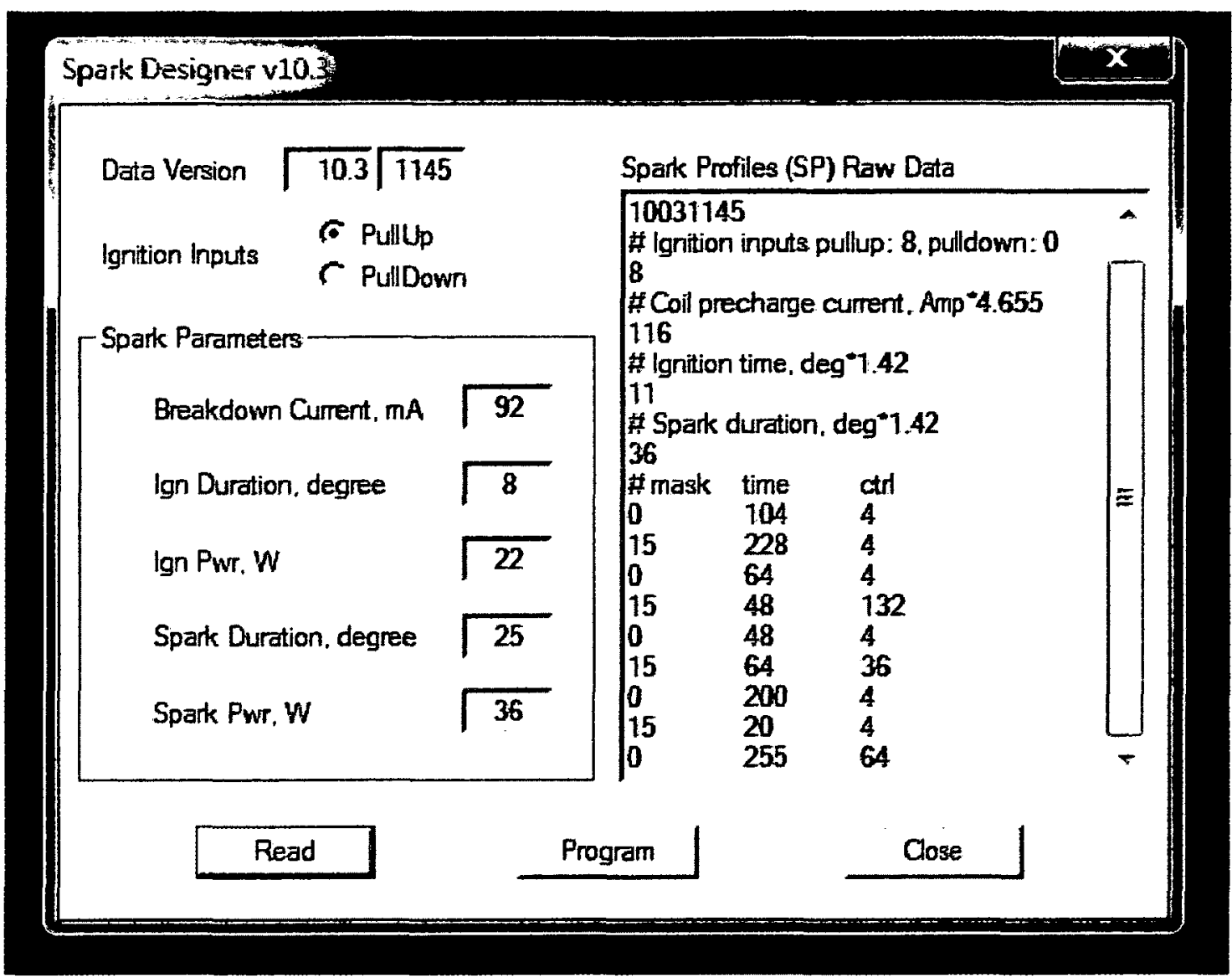

Figure A.2: The user interface for the ignition system and its adjustable parameters; Breakdown Current [mA], Ignition Duration [Deg], Ignition Power [W], Spark Duration [Deg], Spark Power [W] 
Appendix B

\section{EngineWiring}




\section{B.1 Data Acquisition Circuits}

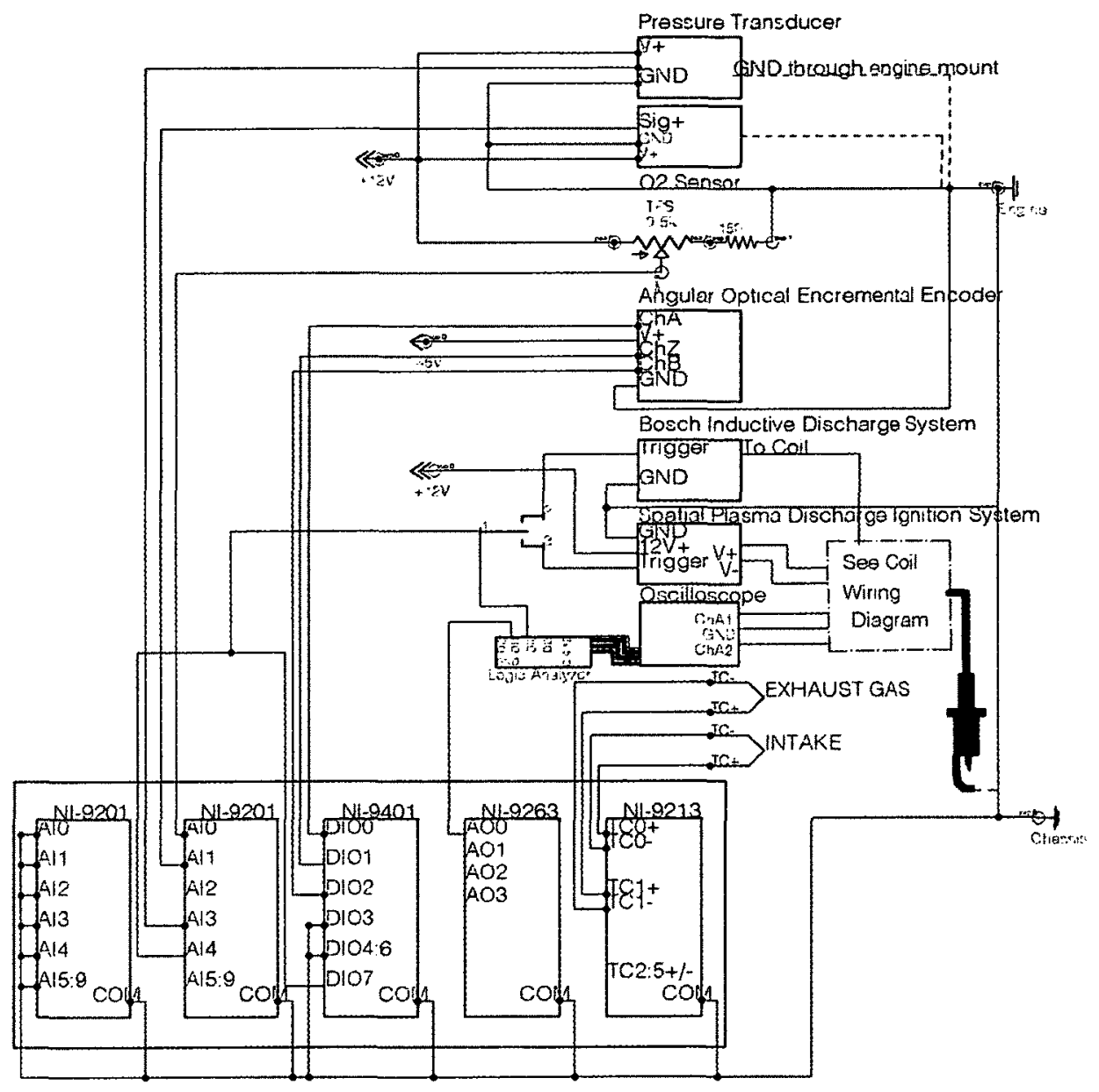

Figure B.1: Diagram of the circuits and ports/lines used for the engine setup and the National instruments data acquisition board array. 


\section{B.2 Coil Circuits}

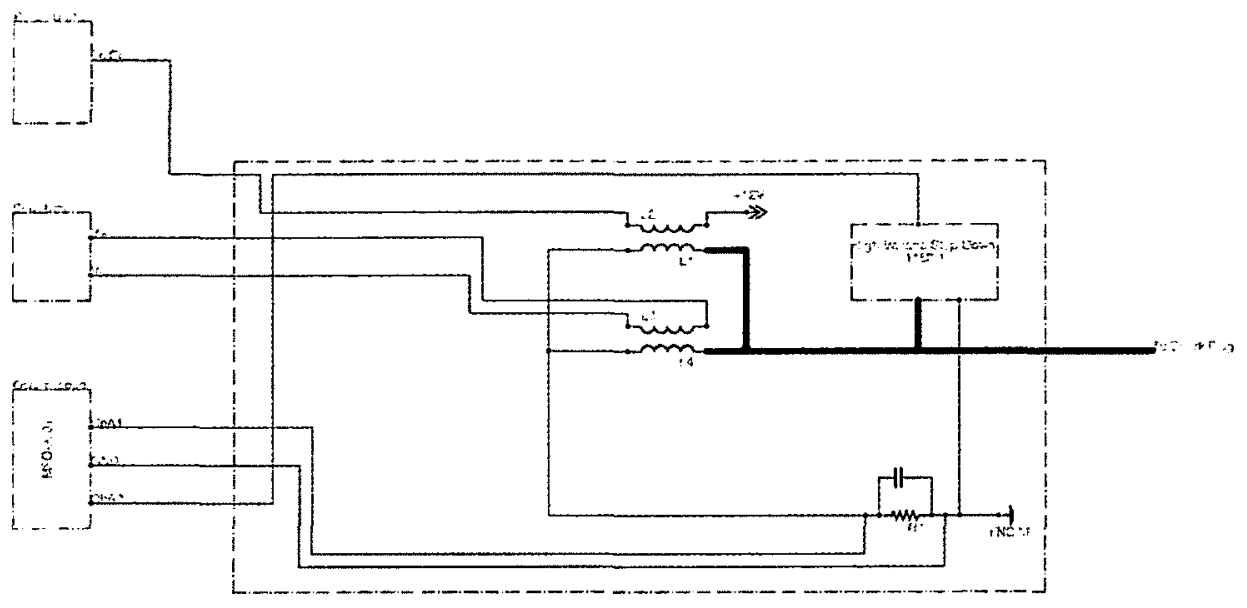

Figure B.2: Schematic of coil arrangements for both SPDI and Bosch Units.

\section{B.3 Power Supply Circuits}

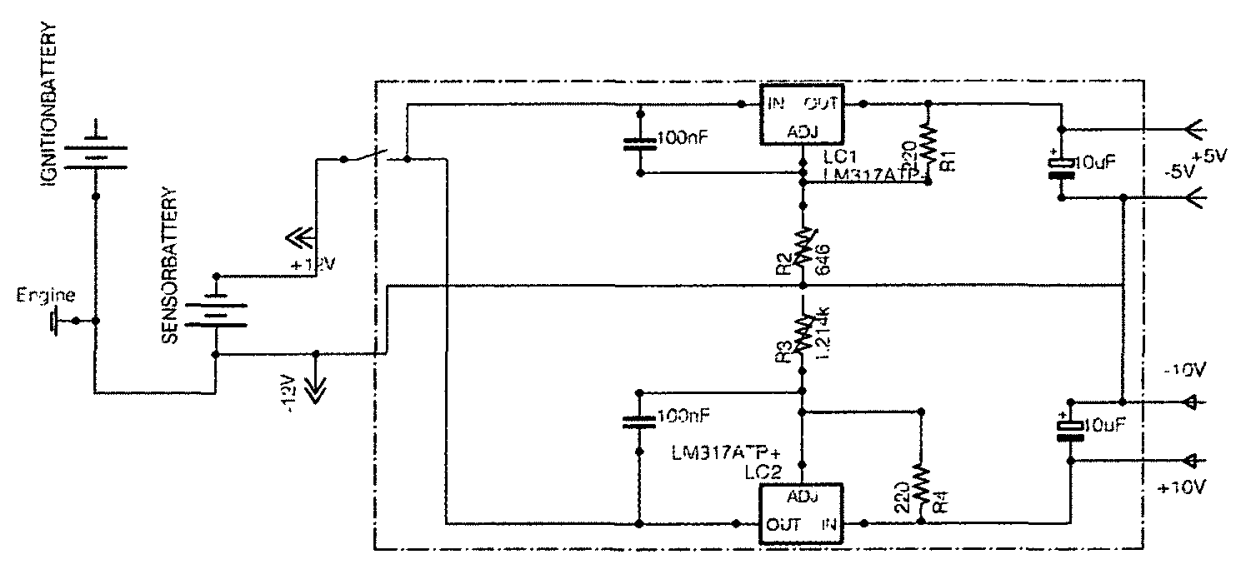

Figure B.3: Schematic of power supply unit made to regulate $12 \mathrm{~V}$ down to $5 \mathrm{~V}$ and $10 \mathrm{~V}$. 


\section{B.4 Physical Coils Used in Acquiring Voltage and Current}

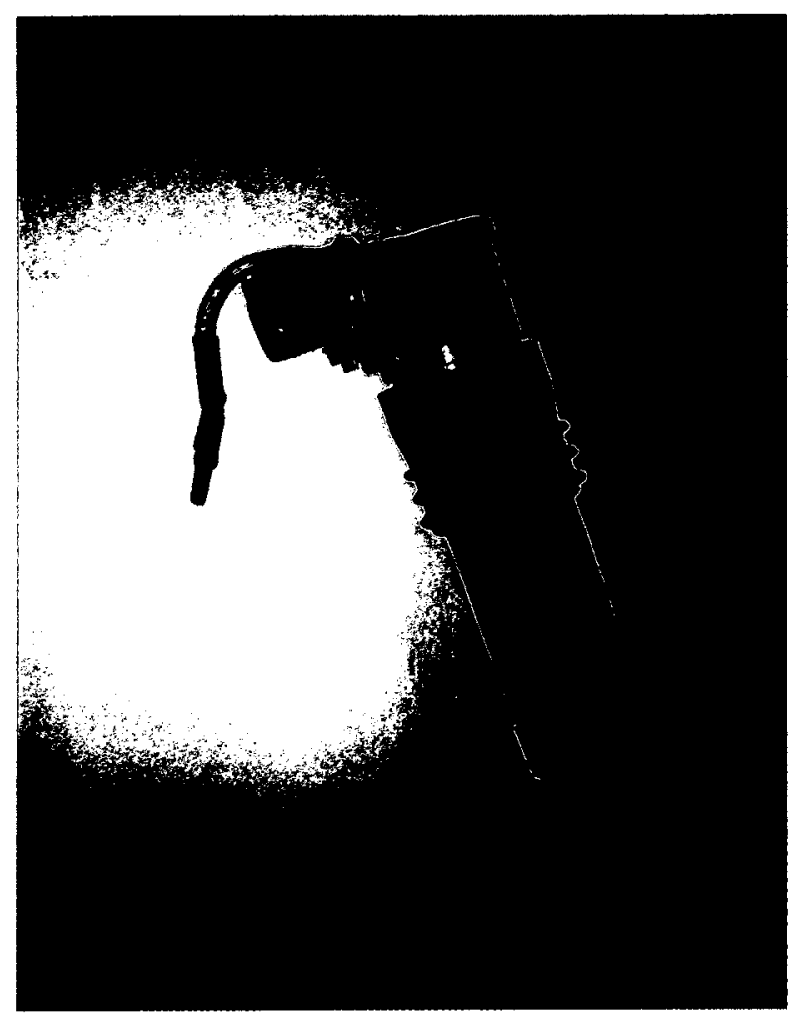

Figure B.4: Image of physical coil used in the experiments. 


\section{Appendix $\mathbf{C}$}

\section{Data Processing}




\section{C.1 MATLAB Flow Chart}

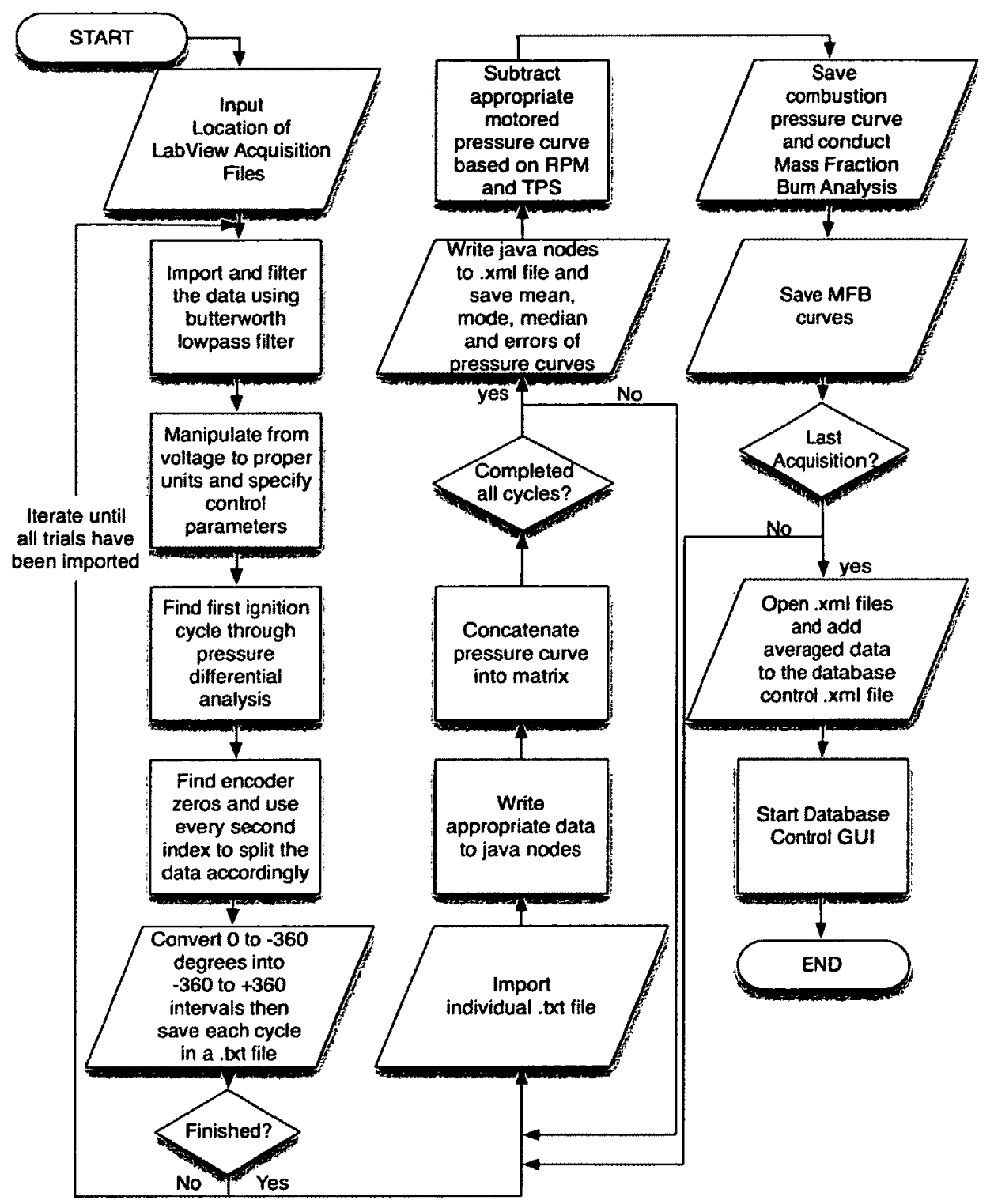

Figure C.1: Flow chart of data analysis and addition to the database. 


\section{C.2 MATLAB Database GUI Front Panel}

\section{C.3 Pressure Filter and Residuals}

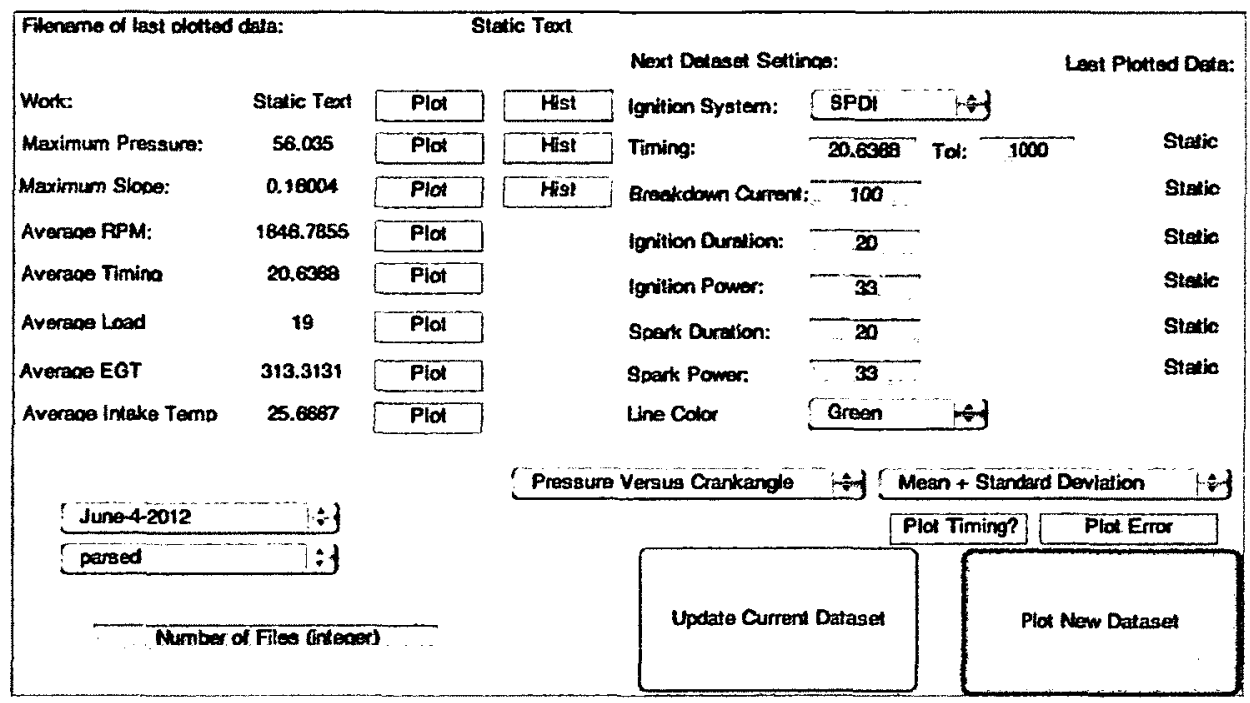

Figure C.2: An image of the front panel used in the MATLAB database evaluation program. 


\section{C.4 Pressure Filter and Residuals}

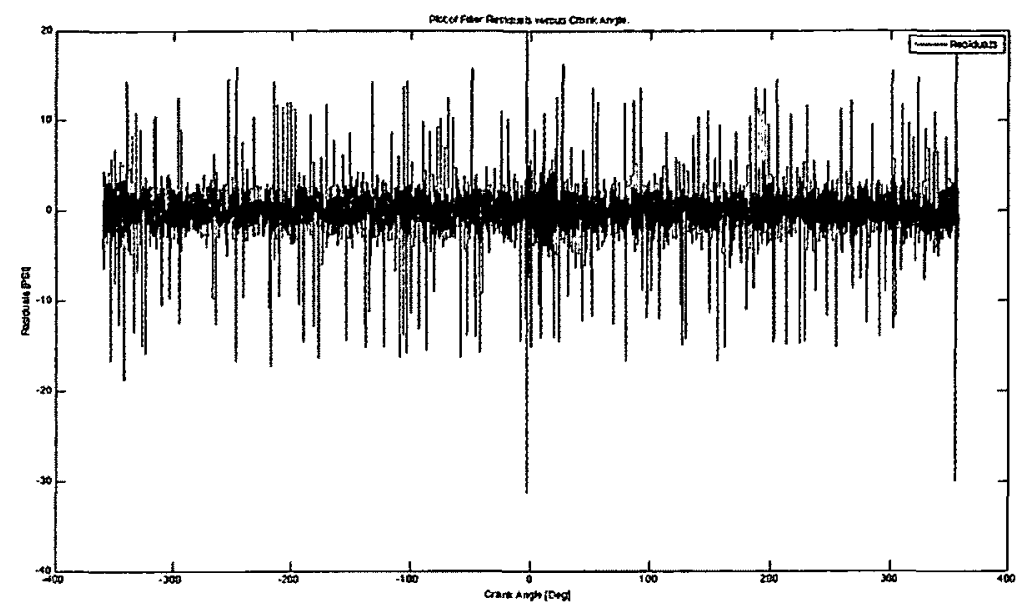

Figure C.3: A plot of the residuals from subtracting the filtered data from the non-filtered data. 


\section{C.5 Statistical Analyses - Supporting Figures}
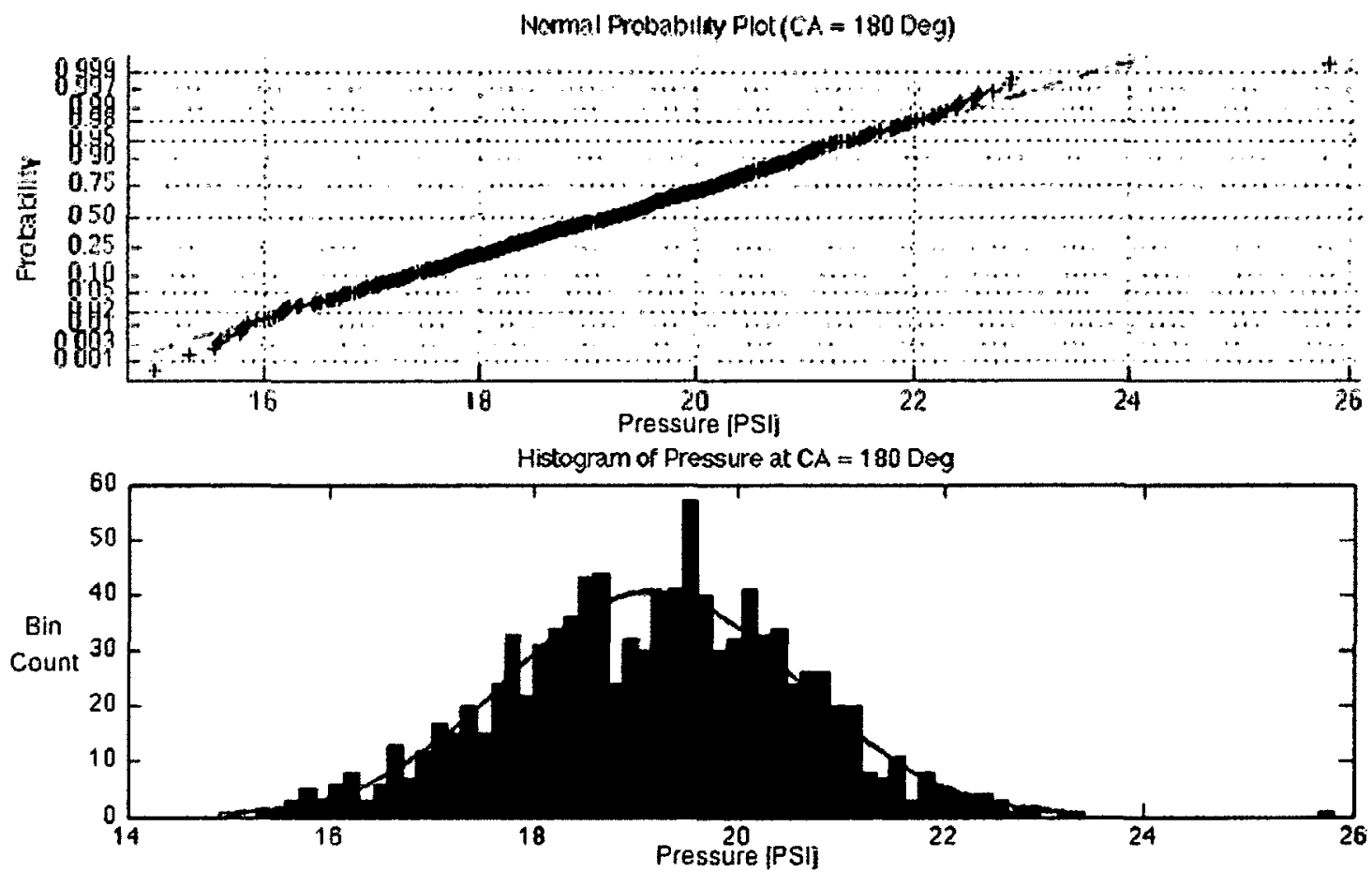

Figure C.4: Histograms and normal probability plot for a single trials cylinder pressures corresponding to 180 degrees crank angle location. 


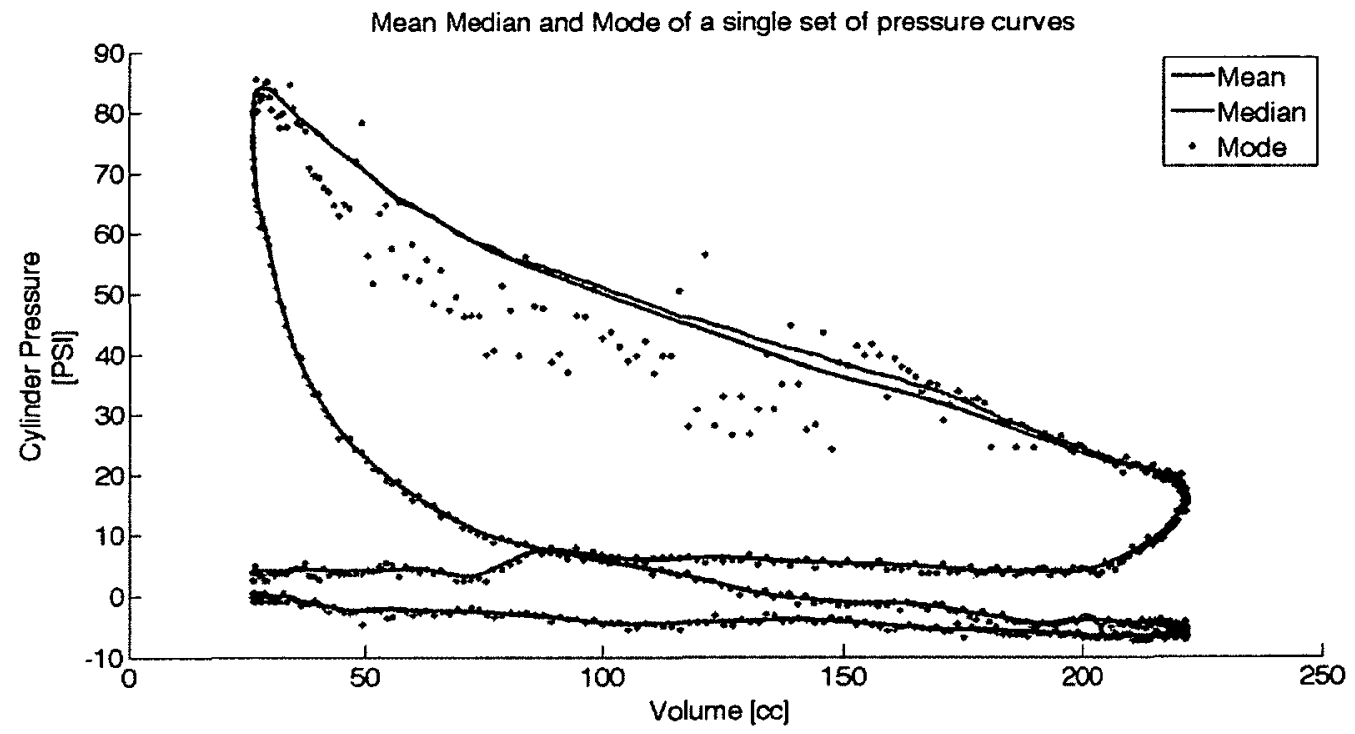

Figure C.5: A plot of the mean, median and mode for a sample pressure trace acquisition inside the combustion chamber of a single cylinder engine. 
Appendix D

Results and Discussion: Supporting

Figures 


\section{D.1 Pressure Traces for Variable Spark Duration}

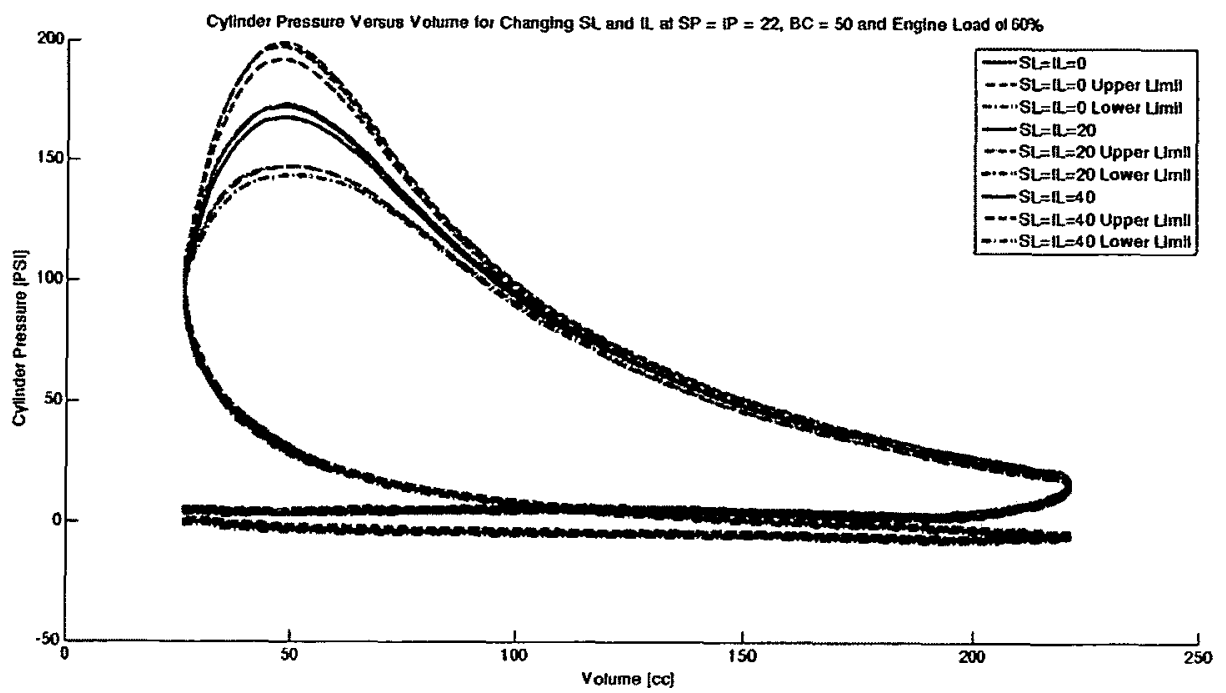

Figure D.1: Cylinder pressure versus volume for three spark durations at SPDI settings of: $\mathrm{BC}=50, \mathrm{SL}=\mathrm{IL}=[0,20,40], \mathrm{SP}=\mathrm{IP}=22$, and engine load of $60 \%$. (see Table 2.1 on page 23 for a description of the SPDI settings) 


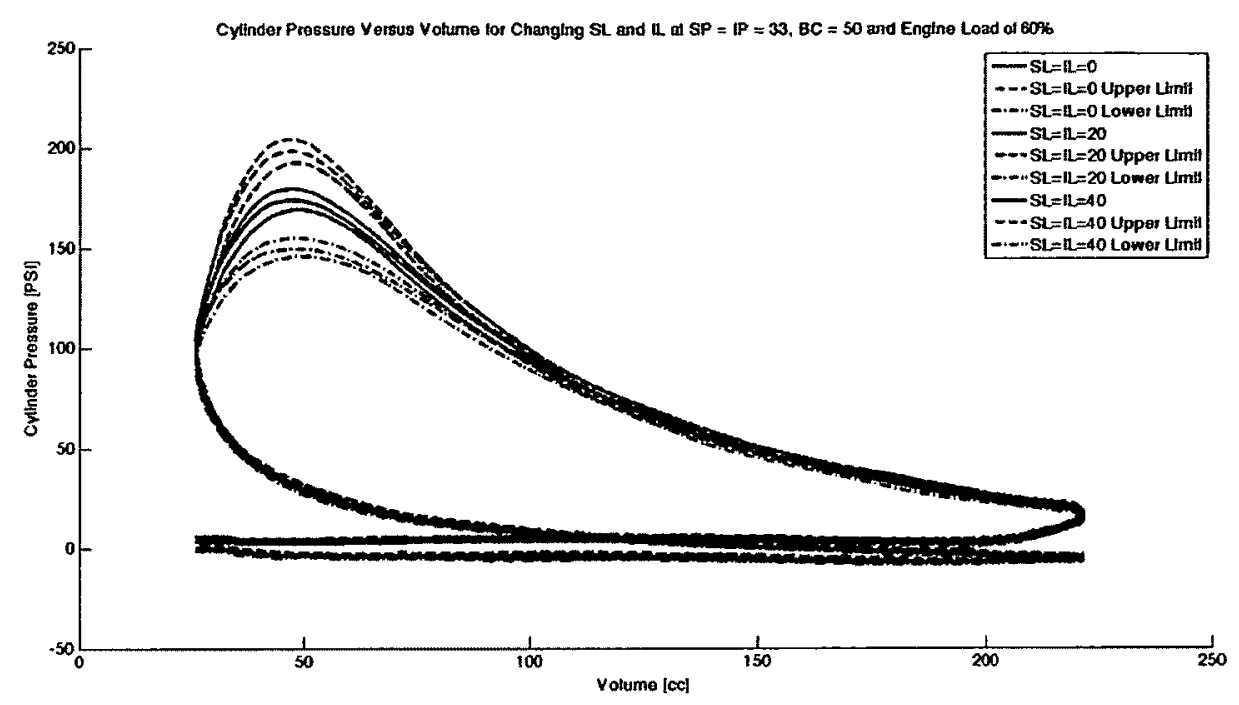

Figure D.2: Cylinder pressure versus volume for three spark durations at SPDI settings of: $\mathrm{BC}=50, \mathrm{SL}=\mathrm{IL}=[0,20,40], \mathrm{SP}=\mathrm{IP}=33$, and engine load of $60 \%$. (see Table 2.1 on page 23 for a description of the SPDI settings)

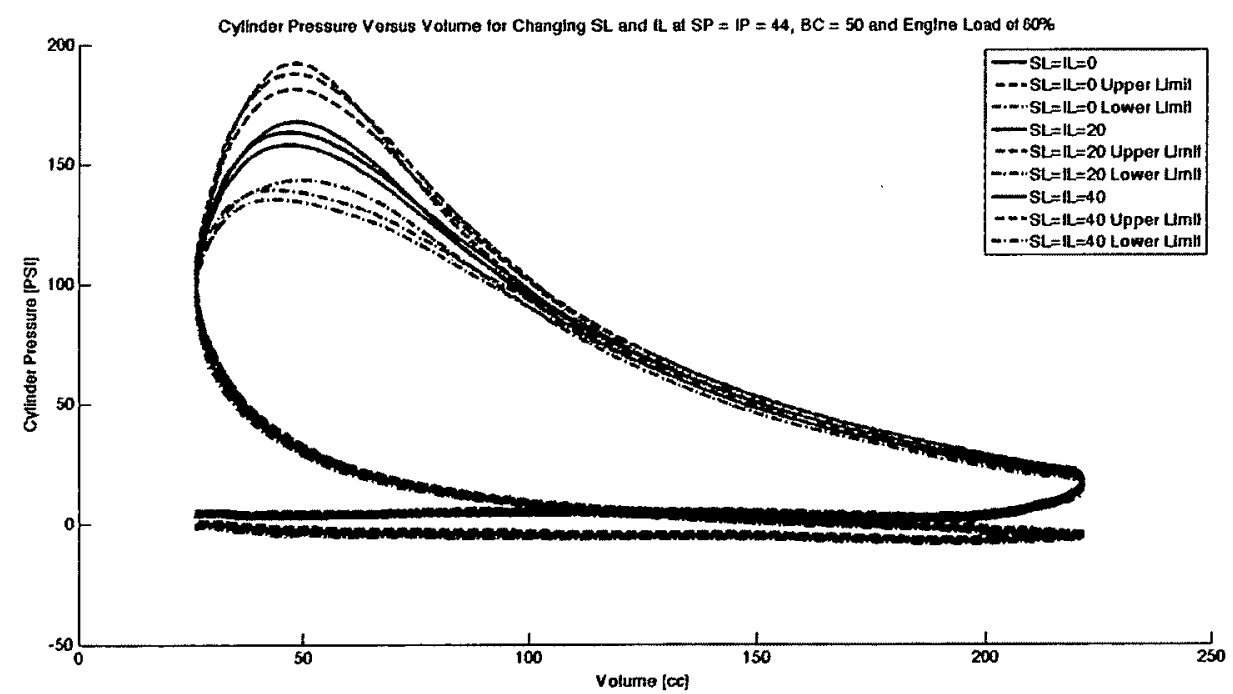

Figure D.3: Cylinder pressure versus volume for three spark durations at SPDI settings of: $\mathrm{BC}=50, \mathrm{SL}=\mathrm{IL}=[0,20,40], \mathrm{SP}=\mathrm{IP}=44$, and engine load of $60 \%$. (see Table 2.1 on page 23 for a description of the SPDI settings) 


\section{D.2 Pressure Traces for Variable Spark Plasma}

\section{Power}

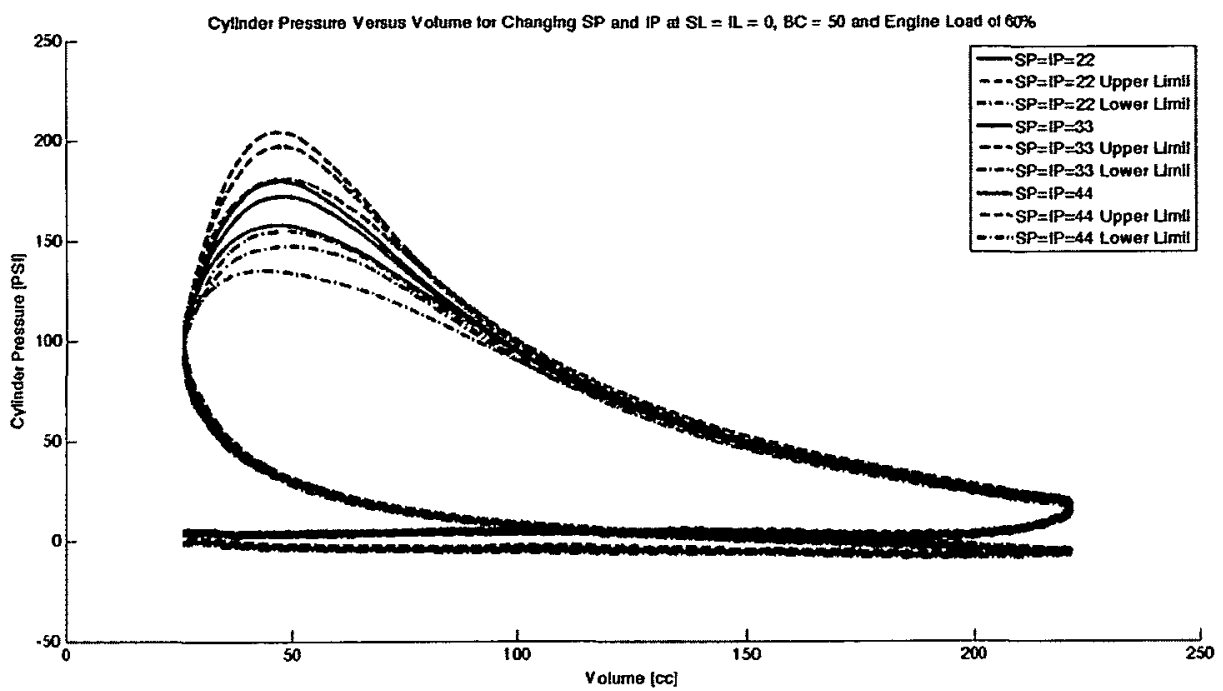

Figure D.4: Cylinder pressure versus crank angle for three spark durations at SPDI settings of: $\mathrm{BC}=50, \mathrm{SL}=\mathrm{IL}=0, \mathrm{SP}=\mathrm{IP}=[22,33,44]$, and engine load of $60 \%$. (see Table 2.1 on page 23 for a description of the SPDI settings) 


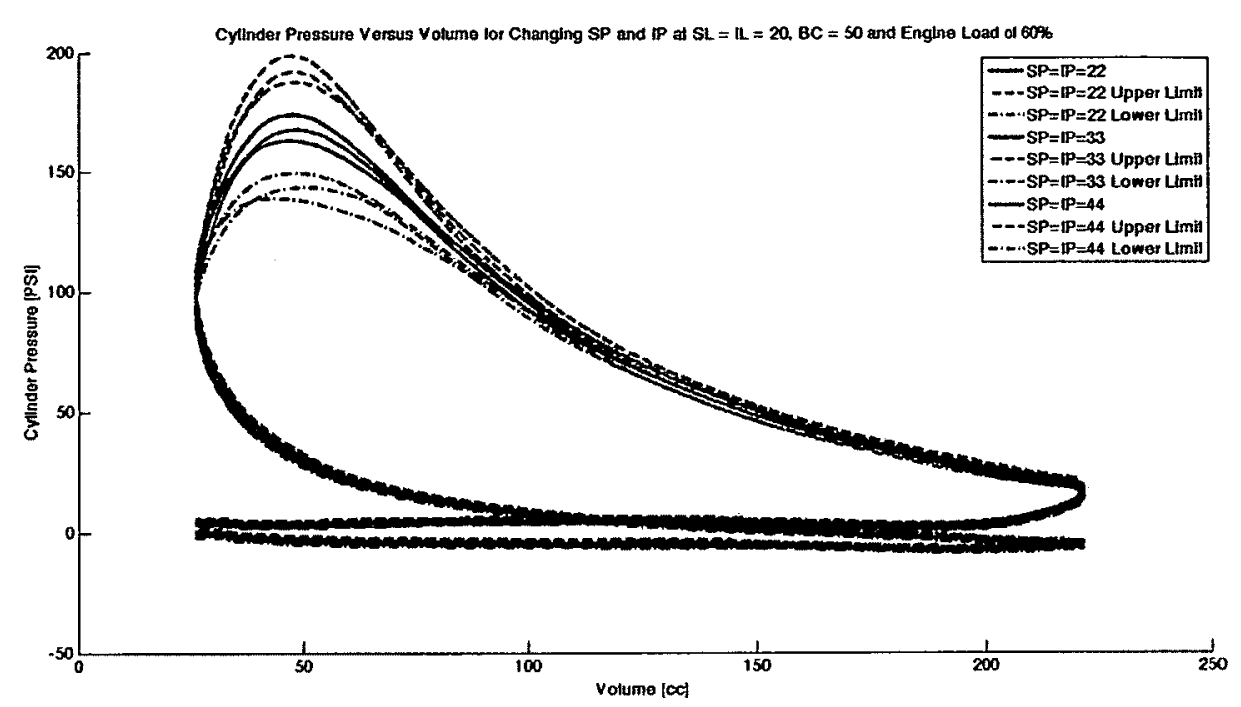

Figure D.5: Cylinder pressure versus crank angle for three spark durations at SPDI settings of: $\mathrm{BC}=50, \mathrm{SL}=\mathrm{IL}=20, \mathrm{SP}=\mathrm{IP}=[22,33,44]$, and engine load of $60 \%$. (see Table 2.1 on page 23 for a description of the SPDI settings)

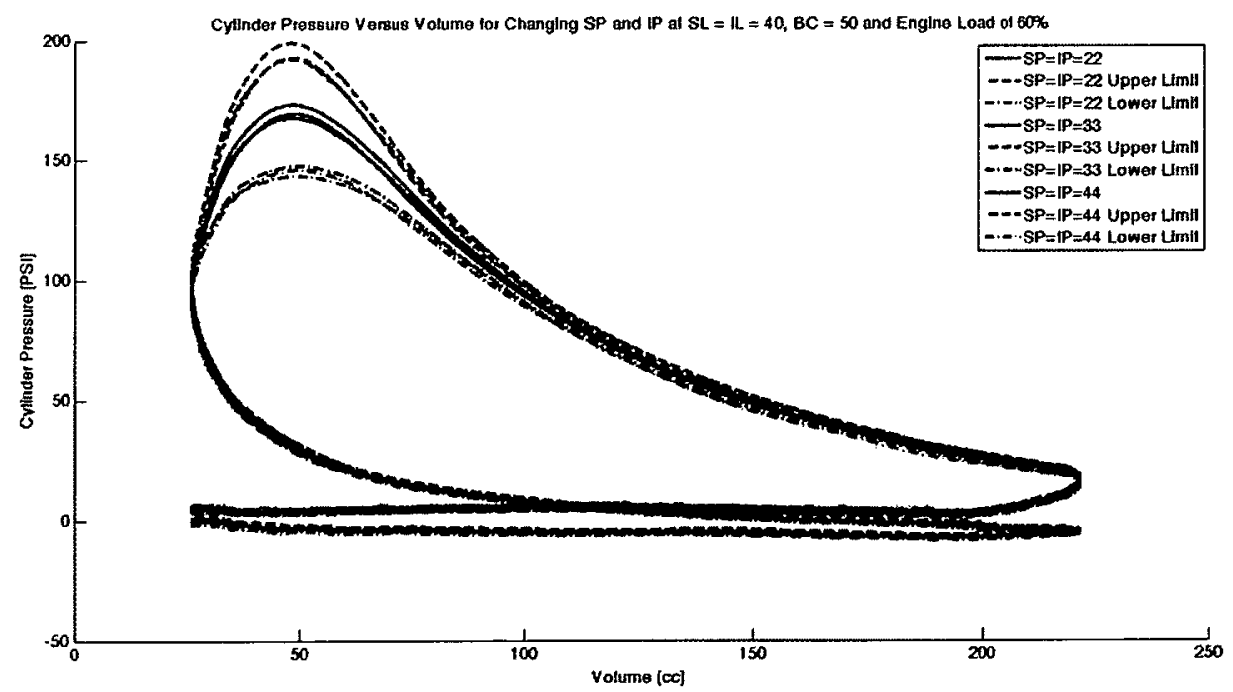

Figure D.6: Cylinder pressure versus crank angle for three spark durations at SPDI settings of: $\mathrm{BC}=50, \mathrm{SL}=\mathrm{IL}=40, \mathrm{SP}=\mathrm{IP}=[22,33,44]$, and engine load of $60 \%$. (see Table 2.1 on page 23 for a description of the SPDI settings) 


\section{D.3 Pressure Traces for Variable Breakdown Cur-}

\section{rent}

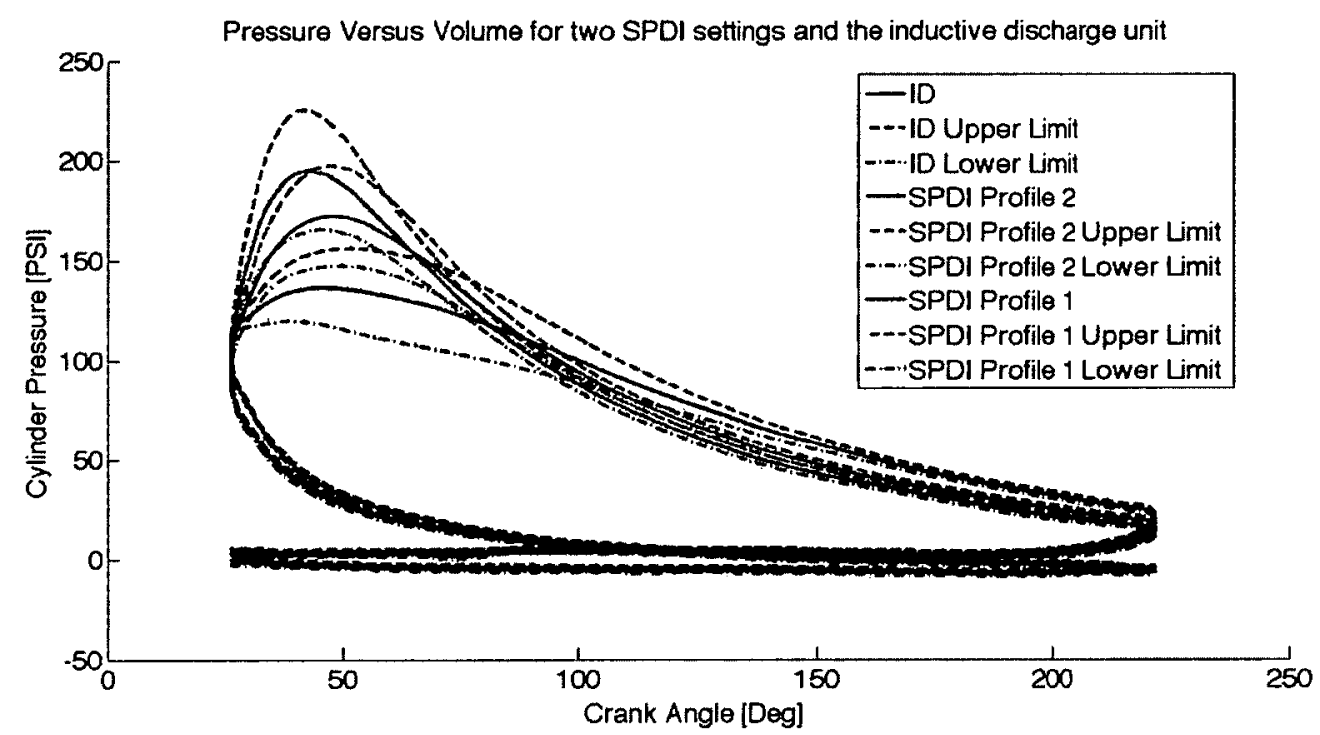

Figure D.7: Cylinder pressure versus crank angle for three trials, two SPDI and one ID at an engine load of $60 \%$. (see Table 2.1 on page 23 for a description of the SPDI settings) 


\section{List of References}

[1] A. Fridman. Plasma Chemistry. Cambridge University Press, New York, New York (2008).

[2] N.Bohr. "On the constitution of atoms and molecules." Philosophical Magazine 26, 1-24 (1913).

[3] "metastable". Oxford Dictionaries. April 2010. Oxford University Press. http://oxforddictionaries.com/definition/english/metastable. (accessed September 27, 2012).

[4] P. J. Mohr, B. N. Taylor, and D. B. Newell. CODATA Recommended Values of the Fundamental Physical Constants: 2010. National Institute of Standards and Technology, Gaithersburg, Maryland 20899-8420, USA (2012).

[5] S. Jockusch, N. J. Turro, E. K. Thompson, M. Gouterman, J. B. Callis, and G. E. Khalil. "Singlet molecular oxygen by direct excitation." Photochemical and Photobiological Sciences 7, 235-239 (2008).

[6] O. Svelto. Principles of Lasers, chapter 6, page 246. Springer, fifth edition edition (2010).

[7] D. Singleton, S. J. Pendleton, and M. A. Gundersen. "The role of non-thermal transient plasma for enhanced flame ignition in $\mathrm{C} 2 \mathrm{H} 4$ - air." Journal of Physics D: Applied Physics 44, 1-6 (2011).

[8] G. Lou, A. Bao, M. Nishihara, S. Keshav, Y. G. Utkin, J. W. Rich, W. R. Lempert, and I. V. Adamovich. "Ignition of premixed hydrocarbon-air flows 
by repetitively pulsed, nanosecond pulse duration plasma." Proceedings of the Combustion Institute 31(2), 3327 - 3334 (2007).

[9] I. N. Kosarev, N. L. Aleksandrov, S. V. Kindysheva, S. M. Starikovskaia, and A. Y. Starikovskii. "Kinetics of ignition of saturated hydrocarbons by nonequilibrium plasma: CH4-containing mixtures." Combustion and Flame pages 569 -586 (2008).

[10] G. Lou, A. Bao, M. Nishihara, S. Keshav, Y. G. Utkin, J. W. Rich, W. R. Lempert, and I. V. Adamovich. "Ignition of premixed hydrocarbon-air flows by repetitively pulsed, nanosecond pulse duration plasma." Proceedings of the Combustion Institute 31(2), 3327 - 3334. ISSN 1540-7489 (2007).

[11] C. Cathey, J. Cain, H. Wang, M. A. Gundersen, C. Carter, and M. Ryan. "OH production by transient plasma and mechanism of flame ignition and propagation in quiescent methane-air mixtures." Combustion and Flame 154(4), 715 727. ISSN 0010-2180 (2008).

[12] A. Starikovskii. "Plasma supported combustion." Proceedings of the Combustion Institute 30(2), 2405 - 2417. ISSN 1540-7489 (2005).

[13] S. M. Starikovskaia. "Plasma assisted ignition and combustion." Journal of Physics D: Applied Physics 39(16), R265 (2006).

[14] L. A. Rosocha, D. M. Coates, D. Platts, and S. Stange. "Plasma-enhanced combustion of propane using a silent discharge." Physics of Plasmas 11(5), 2950-2956 (2004).

[15] R. Ono and T. Oda. "OH radical measurement in a pulsed arc discharge plasma observed by a LIF method." Industry Applications, IEEE Transactions on 37(3), 709 -714. ISSN 0093-9994 (2001).

[16] C. Cathey, T. Tang, T. Shiraishi, T. Urushihara, A. Kuthi, and M. Gundersen. "Nanosecond plasma ignition for improved performance of an internal combustion engine." Plasma Science, IEEE Transactions on 35(6), 1664-1668. ISSN 0093-3813 (2007). 
[17] S. V. Pancheshnyi, D. A. Lacoste, A. Bourdon, and C. O. Laux. "Ignition of propane-air mixtures by a repetitively pulsed nanosecond discharge." Plasma Science, IEEE Transactions on 34(6), 2478 -2487. ISSN 0093-3813 (2006).

[18] L. Wu, J. Lane, N. Cernansky, D. Miller, A. Fridman, and A. Starikovskiy. "Plasma-assisted ignition below self-ignition threshold in methane, ethane, propane and butane-air mixtures." Proceedings of the Combustion Institute 33(2), 3219 - 3224. ISSN 1540-7489 (2011).

[19] T. Shiraishi, T. Urushihara, and M. Gundersen. "A trial of ignition innovation of gasoline engine by nanosecond pulsed low temperature plasma ignition." Journal of Physics D: Applied Physics 42(13), 135208 (2009).

[20] A. Bourig, D. Thevenin, J.-P. Martin, G. Janiga, and K. Zahringer. "Numerical modeling of $\mathrm{H} 2-\mathrm{O} 2$ flames involving electronically-excited species $\mathrm{O}_{2}\left(a^{1} \delta_{g}\right)$, $\mathrm{O}\left({ }^{1} \mathrm{D}\right)$ and $\mathrm{OH}\left({ }^{2} \sigma^{+}\right) . "$ Proceedings of the Combustion Institute 32(2), 3171 3179. ISSN 1540-7489 (2009).

[21] H. Do. Plasma-Assisted Combustion in a Supersonic Flow. Ph.D. thesis, Stanford University (2009).

[22] G. Rassweiler and L. Withrow. "Motion pictures of engine flames correlated with pressure cards." SAE Technical Paper (380139) (1938).

[23] J. K. Ball, R. R. Raine, and C. R. Stone. "Combustion analysis and cycle-bycycle variations in spark ignition engine combustion part 1: an evaluation of combustion analysis routines by reference to model data." Proceedings of the Institution of Mechanical Engineers Part D Journal of Automobile Engineering 212(5), 381-399 (1998).

[24] B. R. Brown. Combustion Data Acquisition and Analysis. Master's thesis, Loughborough University (2001).

[25] J. K. Ball, R. R. Raine, and C. R. Stone. "Combustion analysis and cycle-bycycle variations in spark ignition engine combustion part 2: a new parameter for completeness of combustion and its use in modelling cycle-by-cycle variations in combustion." Proceedings of the Institution of Mechanical Engineers Part D Journal of Automobile Engineering 212(6), 507-523 (1998). 
[26] N. M. Brown. Characterisation of Emissions and Combustion Stability of a Port Fuelled Spark Ignition Engine. Ph.D. thesis, The University of Nottingham (2009).

[27] K. Z. Mendera, A. Spyra, and M. Smereka. "Mass fraction burned analysis." Journal of KONES internal Combustion Engines (3-4), 193-201. ISSN 12314005 (2002).

[28] P. J. Shayler, M. W. Wiseman, and T. Ma. "Improving the determination of mass fraction burnt." SAE Technical Papers 900351 (1990).

[29] H. M. Cheung and J. B. Heywood. "Evaluation of a one-zone burn-rate analysis procedure using production si engine pressure data." SAE Technical Papers 932749 (1993).

[30] M. F. J. Brunt and C. R. Pond. "Evaluation of techniques for absolute cylinder pressure correction." SAE Technical Papers 970036 (1997).

[31] S. Y. Moon, W. Choe, and B. K. Kang. "A uniform glow discharge plasma source at atmospheric pressure." Applied Physics Letters 84(2), 188-190 (2004).

[32] T. Bae, R. A. Atkins, H. F. Taylor, and W. N. Gibler. "Interferometric fiberoptic sensor embedded in a spark plug for in-cylinder pressure measurement in engines." Appl. Opt. 42(6), 1003-1007 (2003).

[33] I. Land, H.B. and C. Eddins. "Optical pressure measurement: using fiber optic transducers in hypersonic flight vehicles." Instrumentation Measurement Magazine, IEEE 7(3), 38 - 45. ISSN 1094-6969 (2004).

[34] T. Yamanaka, M. Esaki, and M. Kinoshita. "Measurement of TDC in engine by microwave technique." Microwave Theory and Techniques, IEEE Transactions on 33(12), 1489 - 1494. ISSN 0018-9480 (1985).

[35] M. Tazerout, O. Le Corre, and S. Rousseau. "TDC determination in IC engines based on the thermodynamic analysis of the temperature-entropy diagram." SAE Technical Papers, International Spring Fuels and Lubricants Meeting ISSN 0148-7191 (1999). 